\title{
A CHARACTERIZATION OF ALL SOLUTIONS TO THE FOUR BLOCK GENERAL DISTANCE PROBLEM*
}

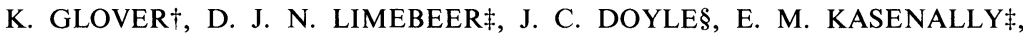 \\ AND M. G. SAFONOVף
}

\begin{abstract}
All solutions to the four block general distance problem which arises in $H^{\infty}$ optimal control are characterized. The procedure is to embed the original problem in an all-pass matrix which is constructed. It is then shown that part of this all-pass matrix acts as a generator of all solutions. Special attention is given to the characterization of all optimal solutions by invoking a new descriptor characterization of all-pass transfer functions. As an application, necessary and sufficient conditions are found for the existence of an $H^{\infty}$ optimal controller. Following that, a descriptor representation of all solutions is derived.
\end{abstract}

Key words. $H^{\infty}$-optimal control, four block problem, Parrott's theorem, general distance problems, indefinite Riccati equations, indefinite factorization, linear quadratic differential games, Nehari's theorem

\section{AMS(MOS) subject classifications.}

1. Introduction. The four block general distance problem has its genesis in certain recent work on $H^{\infty}$ optimal control [7], [10], [14], [15], [43]. In typical $H^{\infty}$ design situations, a nominal plant model is known, and the design engineer has the task of selecting various frequency-dependent weights. The plant model and weights are then combined into a single matrix

$$
P(s)=\left[\begin{array}{ll}
P_{11} & P_{12} \\
P_{21} & P_{22}
\end{array}\right](s)
$$

and we seek to characterize all internally stabilizing controllers which satisfy $\left\|F_{l}(P, K)\right\|_{\infty} \leqq \gamma$. Rather than tackling this (nonlinear) problem directly, it can be converted into another problem which is linear in a free parameter. That is, find all those $Q \in H_{\infty}^{+}$such that

$$
\left\|\left(T_{11}+T_{12} Q T_{21}\right)(s)\right\|_{\infty} \leqq \gamma
$$

in which $T_{11}(s), T_{12}(s)$, and $T_{21}(s)$ may always be chosen stable with $T_{12}(s)$ and $T_{21}(s)$ parts of inner matrices [10], [14], [15], [43], [46]. We change nothing by rewriting (1.2) as

$$
\left\|\left(T_{11}+\left[T_{\perp} T_{12}\right]\left[\begin{array}{ll}
0 & 0 \\
0 & Q
\end{array}\right]\left[\begin{array}{c}
\tilde{T}_{\perp} \\
T_{21}
\end{array}\right]\right)(s)\right\|_{\infty} \leqq \gamma, \quad Q \in \mathscr{H}_{\infty}^{+}
$$

in which $T_{\perp}(s)$ and $\tilde{T}_{\perp}(s)$ are chosen to make $\left[T_{\perp} T_{12}\right](s)$ and $\left[\tilde{T}_{\perp}^{\sim} T_{21}^{\sim}\right]^{\sim}(s)$ inner. This, too, is always possible [2], [10], [16], [43]. Finally, by invoking the norm preserving property of inner matrices, we see that $(1.3)$ is equivalent to the characterization of all $Q \in \mathscr{H}_{\infty}^{+, p \times m}$ such that

$$
\left\|\left[\begin{array}{cc}
R_{11} & R_{12} \\
R_{21} & R_{22}+Q
\end{array}\right](s)\right\|_{\infty} \leqq \gamma
$$

\footnotetext{
* Received by the editors May 16, 1988; accepted for publication (in revised form) January 26, 1990.

$\dagger$ Department of Engineering, University of Cambridge, Trumpington Street, Cambridge CB2 1PZ, United Kingdom.

‡ Department of Electrical Engineering, Imperial College, Exhibition Road, London SW7 2BT, United Kingdom.

$\S$ Department of Electrical Engineering, California Institute of Technology, Pasadena, California 91125.

I Department of Electrical Engineering Systems, University of Southern California, Los Angeles, California 90089-0781.
} 
where

$$
\left[\begin{array}{ll}
\dot{R}_{11} & R_{12} \\
R_{21} & R_{22}
\end{array}\right](s)=\left[\begin{array}{l}
T_{\perp}^{\sim} \\
T_{12}
\end{array}\right] T_{11}\left[\tilde{T}_{\perp}^{\sim} T_{21}^{\sim}\right](s) \in R H_{-}^{\infty} .
$$

In this paper we will study the four block general distance problem given in (1.4) because of its intrinsic interest, and also due to its applicability to $H^{\infty}$ control.

Historical accounts of the development of solutions to this problem are contained in [11], [14] and [43]. Briefly, Doyle et al. [10], originally suggested a solution to the four block problem (1.4) based on the work of Davis et al. [9]. Other approaches included work on Hankel plus Toplitz operators in Jonckheere and Juang, [26] and on band extension problems in Dym and Gohberg [12]. These approaches certainly provide a theoretical solution, but when implemented on a computer, suffer from serious degree inflation problems. In an attempt to understand these inflation phenomena in the context of $H^{\infty}$ control, detailed cancellation analyses were carried out in [30], [31], [32], and [23] for cases of increasing complexity, and a controller with degree no greater than that of $P(s)$ in (1.1) was shown to exist. The outcome of this work also showed that solutions with $\operatorname{deg}(Q) \leqq \operatorname{deg}(R)$ exist to (1.4). These observations lead to the expectation that the original algorithm could be greatly improved and progress was made in [3] where just three Riccati equations of modest degree were required. The purpose of this paper is to present a new solution to the four block general distance problem requiring just two Riccati equations and also treating the optimal cases.

As indicated above, the solutions to the four block general distance problem can give representation formulae for all solutions to the $H^{\infty}$ control problem (1.2). One such formula is given in $\S 5$ where the optimal cases are treated in detail. This solution requires two Riccati equations to be solved or, more precisely, (for certain optimal cases) for the appropriate stable invariant subspaces of two Hamiltonian matrices to be calculated. Solution formulae based on two Riccati equations were presented without proof in [20] for the suboptimal case and subsequently derivations for a limited class of plants were given in [11] using techniques similar to the state feedback results of Khargonekar, Petersen, and Rotea [28]. Formulae for certain optimal cases were given in [33], [35], and [45] using a descriptor representation and giving relations to interpolation. Many alternative and, in several cases, totally independent derivations of these results are now available using a variety of techniques. A solution based on $J$-spectral factorization theory is given in [22], while the related approach based on the notion of conjugation is employed in [29]. Hung has derived a formula in terms of two Riccati equations which deals with certain optimal situations [24], while Verma and Romig have a closed formula for one block problems [48]. An interesting byproduct of this activity has been the discovery of a number of new interconnections. In [28], Khargonekar et al. note a connection between $\mathscr{H}^{\infty}$ control and game theory. The interplay between indefinite factorization and game theory, probably first noticed by Banker [4], has been rediscovered in the more general setting of $\mathscr{H}^{\infty}$ control [20], [22], [40]. The connection between risk sensitive optimal control [49] and game theory, originally discovered by Jacobson in the perfect information case [25], has also received renewed interest in the wider setting of $\mathscr{H}^{\infty}$ control [5], [20] and entropy minimization [21], [38]. Results on the finite horizon, time-varying case are given in [34] and [47], and finally, a solution applicable to distributed systems may be found in [13] and is due to Foias and Tannenbaum.

Section 2 contains a summary of the notation we will use. In $\S 3$ we derive necessary and sufficient conditions for the existence of a suboptimal solution to the four block 
problem, and give a representation formula for all solutions. We treat the optimal case in detail in $\S 4$. Section 5 deals with the application to $\mathscr{H}^{\infty}$ control theory. We derive necessary and sufficient conditions for the existence of a solution, and then give a formula for all solutions. By setting up the analysis in a descriptor framework, we are able to give a simple and complete treatment of all the optimal cases. In the event that $P_{11}(\infty) \neq 0$ and $P_{22}(\infty) \neq 0$, the controller formulae become cumbersome to write down. To obviate this difficulty, we employ the loop shifting transformations introduced in [45] to reduce the general case to a problem in which $P_{11}(\infty)=0$ and $P_{22}(\infty)=0$. We summarize the key findings of this work in the conclusions $(\S 6)$.

2. Notation and Preliminaries. The aim of this short section is to summarize the notation we intend to use; most of it is standard.

\subsection{Notation.}

$\mathbb{R}, \mathbb{R}_{+}, \mathbb{C}$ real, nonnegative, and complex numbers,

$\mathbb{R}(s) \quad$ field of rational functions in $s$ with real coefficients,

$\mathbb{C}_{+}, \mathbb{C}_{-} \quad$ open right (respectively, left) half plane,

$\mathbb{F}^{p \times m} \quad$ set of $p \times m$ matrices with elements in $\mathbb{F}(=\mathbb{R}, \mathbb{C}, \mathbb{R}(s)$ etc. $)$,

$\lambda(A) \quad$ spectrum of a square matrix $A$,

$\lambda_{\max }(A) \quad$ eigenvalue of $A$ with largest modulus,

$A^{\prime} \quad$ complex conjugate transpose of $A \in \mathbb{C}^{p \times m}$ (transpose if $A \in \mathbb{R}^{p \times m}$ ),

$A^{\#} \quad$ generalized inverse,

$A^{\dagger} \quad$ Moore-Penrose inverse,

$A \geqq 0, A>0 \quad A$ is positive semidefinite (respectively, positive definite),

$A \leqq 0, A<0 \quad A$ is negative semidefinite (respectively, negative definite),

$\mathscr{L}_{\infty}^{(p \times m)} \quad$ space of $p \times m$ matrices with entries that are bounded on the $j \omega$-axis (including the point at $\infty$ ),

$\|\cdot\|_{\infty} \quad \mathscr{L}^{\infty}$-norm of matrices in $\mathscr{L}^{\infty}$,

$\mathscr{H}_{\infty}^{+, p \times m}$

subspace of $\mathscr{L}^{\infty} ; p \times m$ matrices which are analytic and bounded in $\mathbb{C}_{+}$,

$\mathscr{H}_{\infty}^{--, p \times m} \quad$ subspace of $\mathscr{L}^{\infty} ; p \times m$ matrices which are analytic and bounded in $\mathbb{C}_{-}$,

$\|\cdot\|_{H} \quad$ Hankel norm,

$\mathscr{R}_{\mathscr{L}_{\infty}^{p \times m}} \quad$ same as $\mathscr{L}^{\infty(p \times m)}$ except elements are taken from $\mathbb{R}^{(p \times m)}(s)$,

$\mathscr{R} \mathscr{H}_{\infty}^{+, p \times m} \quad$ same as $\mathscr{H}_{\infty}^{+,(p \times m)}$ except elements are taken from $\mathbb{R}^{(p \times m)}(s)$,

$\mathscr{R} \mathscr{H}_{\infty}^{-, p \times m} \quad$ same as $\mathscr{H}_{\infty}^{-,(p \times m)}$ except elements are taken from $\mathbb{R}^{(p \times m)}(s)$,

$G^{\sim}(s) \quad G(-\bar{s})^{\prime}$, the para-Hermitian conjugate of $G(s)$,

$\bar{\sigma}(A)=\|A\|_{2}$ the spectral norm of $A$,

$\operatorname{Re} s \quad$ real part of $s$,

$\mathscr{R} \mathscr{H}_{2}\left(\mathscr{R} \mathscr{H}_{2}^{\perp}\right)$ sets of functions $f(s)$ which are analytic in $\mathbb{C}_{+}\left(\mathbb{C}_{-}\right)$such that

$\rho(A) \quad$ spectral radius.

Associated with a transfer function matrix $G(s) \in \mathbb{R}(s)^{p \times m}$ of McMillan degree $\leqq n$ is a state-space realization

$$
G(s)=D+C(s I-A)^{-1} B
$$

where $A \in \mathbb{C}^{n \times n}, B \in \mathbb{C}^{n \times m}, C \in \mathbb{C}^{p \times n}$, and $D \in \mathbb{C}^{p \times m}$. We will use the alternative notation $G(s) \stackrel{s}{=}(A, B, C, D)$ or

$$
G(s) \stackrel{s}{=}\left[\begin{array}{c|c}
A & B \\
\hline C & D
\end{array}\right]
$$


for realizations of $G(s)$. Generalized state-space models or descriptor system models [37] give rise to transfer functions via $G(s)=D+C(s E-A)^{-1} B \stackrel{s}{=}(A, B, C, D, E)$.

In the above notation, we have $G^{\sim}(s) \stackrel{s}{=}\left(-A^{\prime}, C^{\prime},-B^{\prime}, D^{\prime}\right)$ and in the case that $D$ is nonsingular, we have $G^{-1}(s) \stackrel{s}{=}\left(A-B D^{-1} C, B D^{-1},-D^{-1} C, D^{-1}\right)$. The system zeros of $G(s)$ are given by $\left\{\lambda\left(A-B D^{-1} C\right)\right\} \supseteq\{$ McMillan zeros of $G(s)\}$; these sets are equal if the realization is minimal. If $G^{-1}(s)=G^{\sim}(s)$, then $G(s)$ is all-pass. $G(s)$ is called stable if all its poles are in $\mathbb{C}_{-}$.

We will talk about basis changes $T$ in the state-space of $G(s)$; we will take this to mean $G(s) \stackrel{s}{=}(A, B, C, D) \stackrel{M}{\rightarrow} G(s) \stackrel{s}{=}\left(T A T^{-1}, T B, C T^{-1}, D\right)$. For descriptor system models, basis changes are given by

$$
G(s) \stackrel{s}{=}(A, B, C, D, E) \stackrel{U, V}{\longrightarrow} G(s) \stackrel{s}{=}(U A V, U B, C V, D, U E V) .
$$

We shall also make use of linear fractional transformations which are defined by

$$
F_{l}\left\{\left[\begin{array}{ll}
H_{11} & H_{12} \\
H_{21} & H_{22}
\end{array}\right], U\right\}=H_{11}+H_{12} U\left(I-H_{22} U\right)^{-1} H_{21}
$$

where $U$ is of dimension $l \times m$ if $H_{22}$ has dimension $m \times l$.

3. Construction of an all-pass embedding. In this section we derive necessary and sufficient conditions for the existence of a $Q_{22}(s) \in \mathscr{R} \mathscr{H}_{\infty}^{+}$such that

$$
\left\|\left[\begin{array}{cc}
R_{11} & R_{12} \\
R_{21} & R_{22}+Q_{22}
\end{array}\right]\right\|_{\infty}<1
$$

for given $R_{i j}(s) \in \mathscr{R} \mathscr{H}_{\infty}^{-, p_{i} \times m_{j}}$, and necessary conditions for the existence of $Q_{22}(s) \in \mathscr{R} \mathscr{H}_{\infty}^{+}$ such that

$$
\left\|\left[\begin{array}{cc}
R_{11} & R_{12} \\
R_{21} & R_{22}+Q_{22}
\end{array}\right]\right\|_{\infty} \leqq 1
$$

For simplicity, we will assume that

$$
\bar{\sigma}\left(\left[R_{11}(\infty), R_{12}(\infty)\right]\right)<1
$$

and

$$
\bar{\sigma}\left(\left[\begin{array}{l}
R_{11}(\infty) \\
R_{21}(\infty)
\end{array}\right]\right)<1 .
$$

If $\bar{\sigma}\left(\left[R_{11}(j \omega), R_{12}(j \omega)\right]\right)<1$ and $\bar{\sigma}\left(\left[R_{11}^{\sim}(j \omega), R_{21}^{\sim}(j \omega)\right]\right)<1$ for some $\omega$, but not at $\omega=\infty$, then a bilinear transformation of the half plane into the half plane can give an equivalent problem satisfying (3.3) and (3.4). If however $\bar{\sigma}\left(\left[R_{11}(j \omega), R_{12}(j \omega)\right]\right)=1$ for all $\omega$ or $\bar{\sigma}\left(\left[R_{11}^{\sim}(j \omega), R_{21}^{\sim}(j \omega)\right]\right)=1$ for all $\omega$, then significant modifications in detail need to be performed and so this case is not treated here in the interests of brevity and clarity.

Necessity of the conditions will be derived by assuming such a $Q_{22}(s)$ exists and finding an all-pass dilation of (3.2) to the following special structure, which is used to preserve the integrity of the first row and column of (3.1) and (3.2):

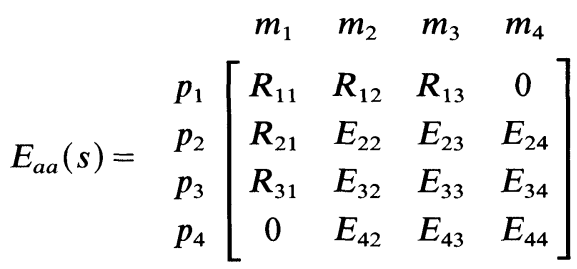

where $E_{i j}=R_{i j}+Q_{i j}, i, j=2,3,4, Q_{i j}(s) \in \mathscr{R} \mathscr{H}_{\infty}^{+, p_{i} \times m_{j}}, R_{i j}(s) \in \mathscr{R} \mathscr{H}_{\infty}^{-, p_{i} \times m_{j}}$. With the allpass dilation constructed in a particular way (Lemma 3.1), so that $R_{13}, R_{31}, E_{24}^{\sim}, E_{42}^{\sim}$ 
being full rank in $\mathbb{C}_{-}$(i.e., $R_{13}$ and $R_{31}$ are minimum phase), it turns out that (3.2) implies that (Proposition 3.2)

$$
\left\|R_{a}^{\sim}\right\|_{H} \leqq 1
$$

where

$$
R_{a}:=\left[\begin{array}{lll}
R_{11} & R_{12} & R_{13} \\
R_{21} & R_{22} & R_{23} \\
R_{31} & R_{32} & R_{33}
\end{array}\right]
$$

and $R_{a}$ does not depend on $Q_{22}$. Similarly, it will be shown in Proposition 3.3 that (3.1) implies that

$$
\left\|R_{a}^{\sim}\right\|_{H}<1
$$

The sufficiency of (3.8) is derived in $\S 3.2$ via a state-space construction along the lines of Glover [17], [18]. Finally, all solutions are derived from this particular solution of (3.1).

3.1. Necessary conditions. We now construct the all-pass dilation of (3.2).

LemMA 3.1. Suppose there exists $Q_{22}(s) \in \mathscr{R} \mathscr{H}_{\infty}^{+}$such that (3.2) holds with $R_{i j}$ satisfying (3.3) and (3.4). Then there exists an all-pass dilation in the form (3.5) where

$$
\begin{aligned}
& E_{a a} E_{a a}^{\sim}=E_{a a}^{\sim} E_{a a}=I, \\
& m_{3}=p_{1}, \quad m_{4} \leqq p_{2}, \quad p_{3}=m_{1}, \quad p_{4} \leqq m_{2}, \\
& R_{13} \in \mathscr{R}_{\infty}^{-}, \quad \text { rank } R_{13}=p_{1} \quad \forall \operatorname{Re} s<0 \text {, } \\
& R_{31} \in \mathscr{R} \mathscr{H}_{\infty}^{-}, \quad \operatorname{rank} R_{31}=m_{1} \quad \forall \operatorname{Re} s<0 \text {, } \\
& E_{23}:=-\left[\begin{array}{ll}
R_{21} & E_{22}
\end{array}\right]\left[\begin{array}{ll}
R_{11} & R_{12}
\end{array}\right]^{\sim}\left(R_{13}^{-1}\right)^{\sim}, \\
& E_{32}:=-\left(R_{31}^{-1}\right)^{\sim}\left[\begin{array}{ll}
R_{11}^{\sim} & R_{21}^{\sim}
\end{array}\right]\left[\begin{array}{l}
R_{12} \\
E_{22}
\end{array}\right], \\
& \operatorname{rank} E_{24}=m_{4} \quad \forall \operatorname{Re} s>0, \\
& \text { rank } E_{42}=p_{4} \quad \forall \operatorname{Re} s>0 \text {, } \\
& {\left[\begin{array}{ll}
E_{33} & E_{34} \\
E_{43} & E_{44}
\end{array}\right]:=-\left[\begin{array}{cc}
R_{31} & E_{32} \\
0 & E_{42}
\end{array}\right]\left[\begin{array}{ll}
R_{11}^{\sim} & R_{21}^{\sim} \\
R_{12}^{\sim} & E_{22}^{\sim}
\end{array}\right]\left[\begin{array}{cc}
\left(R_{13}^{-1}\right)^{\sim} & -\left(R_{13}^{-1}\right)^{\sim} E_{23}^{\sim}\left(E_{24}^{l}\right)^{\sim} \\
0 & \left(E_{24}^{l}\right)^{\sim}
\end{array}\right]}
\end{aligned}
$$

where $E_{24}^{l} E_{24}=I$, with $E_{24}^{l}$ analytic in $\operatorname{Re} s>0$.

Proof. First let $R_{13}^{\sim}$ and $R_{31}^{\sim}$ be stable minimum phase spectral factors satisfying

$$
\begin{array}{ll}
R_{13} R_{13}^{\sim}=I-R_{11} R_{11}^{\sim}-R_{12} R_{12}^{\sim} \geqq 0 & \forall s=j \omega, \\
R_{31}^{\sim} R_{31}=I-R_{11}^{\sim} R_{11}-R_{21}^{\sim} R_{21} \geqq 0 & \forall s=j \omega
\end{array}
$$

where the nonnegativity follows from (3.2). Furthermore, (3.3) implies rank $R_{13}(\infty)=p_{1}$ and (3.4) implies rank $R_{31}(\infty)=m_{1}$, hence $R_{13}$ and $R_{31}$ are square with $R_{13}^{-1}$ and $R_{31}^{-1}$ analytic in $\operatorname{Re} s<0$. Relations (3.2) and (3.9) also imply that

$$
0 \leqq I-\left[\begin{array}{ll}
R_{11} & R_{12} \\
R_{21} & E_{22}
\end{array}\right]\left[\begin{array}{cc}
R_{11}^{\sim} & R_{21}^{\sim} \\
R_{12}^{\sim} & E_{22}^{\sim}
\end{array}\right]=\left[\begin{array}{cc}
R_{13} R_{13}^{\sim} & R_{13} E_{23}^{\sim} \\
E_{23} R_{13}^{\sim} & I-R_{21} R_{21}^{\sim}-E_{22} E_{22}^{\sim}
\end{array}\right]
$$

yielding

$$
E_{23}=-\left(R_{21} R_{11}^{\sim}+E_{22} R_{12}^{\sim}\right)\left(R_{13}^{\sim}\right)^{-1} .
$$


The second row of (3.5) may now be completed as follows:

$$
E_{24} E_{24}^{\sim}:=I-R_{21} R_{21}^{\sim}-E_{22} E_{22}^{\sim}-E_{23} E_{23}^{\sim} \geqq 0 .
$$

Similarly,

$$
\begin{aligned}
& E_{32}=-\left(R_{31}^{\sim}\right)^{-1}\left(R_{11}^{\sim} R_{12}+R_{21}^{\sim} E_{22}\right), \\
& E_{42}^{\sim} E_{42}:=I-R_{12}^{\sim} R_{12}-E_{22}^{\sim} E_{22}-E_{23}^{\sim} E_{23} \geqq 0 .
\end{aligned}
$$

It is then simply verified that (3.17) completes the dilation.

Now let us examine the partially agumented system

$$
E_{a}:=\left[\begin{array}{lll}
R_{11} & R_{12} & R_{13} \\
R_{21} & E_{22} & E_{23} \\
R_{31} & E_{32} & E_{33}
\end{array}\right]
$$

in more detail. First, note that since $E_{a a}$ is all-pass by Lemma 3.1, that

$$
\left\|E_{a}\right\|_{\infty} \leqq 1 \text {. }
$$

Also by (3.17) and (3.9)

$$
\begin{aligned}
E_{33} & =-\left[\begin{array}{ll}
R_{31} & E_{32}
\end{array}\right]\left[\begin{array}{ll}
R_{11} & R_{12}
\end{array}\right]^{\sim}\left(R_{13}^{-1}\right)^{\sim} \\
& =-\left(R_{31}^{-1}\right)^{\sim}\left[\begin{array}{ll}
I-R_{11}^{\sim} R_{11}-R_{21}^{\sim} R_{21} & -R_{11}^{\sim} R_{12}-R_{21}^{\sim} E_{22}
\end{array}\right]\left[\begin{array}{ll}
R_{11} & R_{12}
\end{array}\right]^{\sim}\left(R_{13}^{-1}\right)^{\sim} \\
& =-\left(R_{31}^{-1}\right)^{\sim} R_{11}^{\sim}\left(R_{13}^{-1}\right)^{\sim}+\left(R_{31}^{-1}\right)^{\sim}\left[\begin{array}{ll}
R_{11}^{\sim} & R_{21}^{\sim}
\end{array}\right]\left[\begin{array}{ll}
R_{11} & R_{12} \\
R_{21} & E_{22}
\end{array}\right]\left[\begin{array}{l}
R_{11}^{\sim} \\
R_{12}^{\sim}
\end{array}\right]\left(R_{13}^{-1}\right)^{\sim} .
\end{aligned}
$$

Hence,

$$
E_{a}=T_{1}\left[\begin{array}{ll}
R_{11} & R_{12} \\
R_{21} & E_{22}
\end{array}\right] T_{2}+T_{3}
$$

where

$$
\begin{gathered}
T_{1}=\left[\begin{array}{cc}
I & 0 \\
0 & I \\
-\left(R_{31}^{-1}\right)^{\sim} R_{11}^{\sim} & -\left(R_{31}^{-1}\right)^{\sim} R_{21}^{\sim}
\end{array}\right], \\
T_{2}=\left[\begin{array}{lll}
I & 0 & -R_{11}^{\sim}\left(R_{13}^{-1}\right)^{\sim} \\
0 & I & -R_{12}^{\sim}\left(R_{13}^{-1}\right)^{\sim}
\end{array}\right], \\
T_{3}=\left[\begin{array}{ccc}
0 & 0 & \left(R_{13}^{-1}\right)^{\sim} \\
0 & 0 & 0 \\
\left(R_{31}^{-1}\right)^{\sim} & 0 & -\left(R_{31}^{-1}\right)^{\sim} R_{11}^{\sim}\left(R_{13}^{-1}\right)^{\sim}
\end{array}\right] .
\end{gathered}
$$

(3.30) Note that $T_{1}, T_{2}$, and $T_{3}$ are analytic in $\operatorname{Re} s>0$.

Relation (3.26) implies that

$$
E_{a}=T_{1}\left[\begin{array}{ll}
R_{11} & R_{12} \\
R_{21} & R_{22}
\end{array}\right] T_{2}+T_{1}\left[\begin{array}{cc}
0 & 0 \\
0 & Q_{22}
\end{array}\right] T_{2}+T_{3} .
$$

Now define $R_{23}, R_{32}$, and $R_{33}$ such

$$
\begin{aligned}
R_{a} & :=\left[\begin{array}{lll}
R_{11} & R_{12} & R_{13} \\
R_{21} & R_{22} & R_{23} \\
R_{31} & R_{32} & R_{33}
\end{array}\right] \\
& =(\text { constant })+\left\{T_{1}\left[\begin{array}{ll}
R_{11} & R_{12} \\
R_{21} & R_{22}
\end{array}\right] T_{2}\right\}_{\text {anticausal }} .
\end{aligned}
$$


That is,

$$
E_{a}=R_{a}+T_{4} Q_{22} T_{5}+T_{6}
$$

where $T_{4}=T_{1}\left[\begin{array}{ll}0 & I\end{array}\right]^{\prime} ; T_{5}=\left[\begin{array}{ll}0 & I\end{array}\right] T_{2} ;\left(T_{4} Q_{22} T_{5}+T_{6}\right) \in \mathscr{R} \mathscr{H}_{\infty}^{+}$since $T_{4}, T_{5}, T_{6}, Q_{22}$ are analytic in $\operatorname{Re}(s)>0$ and $\left\|E_{a}\right\|_{\infty} \leqq 1$ implies that $\left(T_{4} Q_{22} T_{5}+T_{6}\right)$ has no poles on the imaginary axis. Hence (3.24), (3.33) and Nehari's theorem give that $\left\|R_{a}^{\sim}\right\|_{H} \leqq 1$.

The important observation here is that $R_{a}$ can be constructed without explicit knowledge of $Q_{22}$. Moreover, $R_{11}, R_{12}, R_{13}, R_{21}, R_{22}, R_{31}$ uniquely determine $R_{a}$. The following proposition has thus been established.

PROPOSITION 3.2. If there exists $Q_{22} \in \mathscr{R} \mathscr{H}_{\infty}^{+}$such that (3.2) holds with $R_{i j}$ satisfying (3.3) and (3.8), then

$$
\begin{gathered}
\left\|R_{11} \quad R_{12}\right\|_{\infty} \leqq 1 \\
\left\|\begin{array}{l}
R_{11} \\
R_{21}
\end{array}\right\|_{\infty} \leqq 1
\end{gathered}
$$

and

$$
\left\|R_{a}^{\sim}\right\|_{H} \leqq 1
$$

where $R_{a}$ is given in (3.32).

For the case of strict inequality in (3.1), all the above inequalities can be made strict and this is now proven.

Proposition 3.3. If there exists $Q_{22} \in \mathscr{R} \mathscr{H}_{\infty}^{+}$such that (3.1) holds with $R_{i j}$ satisfying (3.3) and (3.4), then

$$
\begin{gathered}
\left\|R_{11} \quad R_{12}\right\|_{\infty}<1 \\
\left\|\begin{array}{l}
R_{11} \\
R_{21}
\end{array}\right\|_{\infty}<1
\end{gathered}
$$

and

$$
\left\|R_{a}^{\sim}\right\|_{H}<1
$$

where $R_{a}$ is given in (3.32).

Proof. Formulae (3.37) and (3.38) are immediate consequences of (3.1) and also imply that $\mathrm{R}_{13}, \mathrm{R}_{31}$ may be chosen with inverses in $\mathscr{R} \mathscr{H}_{\infty}^{-}$. Note that in the expression for $E_{a}$ given in (3.33), $R_{a}, T_{4}, T_{5}$, and $T_{3}$ are all independent of $Q_{22}$. Furthermore, for $\Delta \in \mathscr{R} \mathscr{H}_{\infty}^{+}$, define

$$
E_{\alpha}(\Delta):=R_{a}+T_{4}\left(Q_{22}+\Delta\right) T_{5}+T_{6} .
$$

Then $\left\|E_{a}(\Delta)\right\|_{\infty} \leqq 1$ for all $\Delta$ such that

$$
\|\Delta\|_{\infty}<1-\left\|\begin{array}{ll}
R_{11} & R_{12} \\
R_{21} & E_{22}
\end{array}\right\|_{\infty}
$$

Since the construction of Lemma 3.1 will still work, we will now suppose that $\left\|R_{a}^{\sim}\right\|_{H}=1$ and construct a contradiction. For all $\Delta$ satisfying (3.41), we have

$$
E_{a}(\Delta) V(s)=U(-s)
$$

where $V$ and $U$ are the Laplace transforms of the corresponding Schmidt vectors for the Hankel operator corresponding to $R_{a}^{-}$[14], [36]. Note that $U, V \in \mathscr{R} \mathscr{H}_{2}$. Hence $\left(E_{a}(\Delta)-E_{a}(0)\right) V(s)=0 \Rightarrow T_{4} \Delta T_{5} V(s)=0$ for all $\Delta$ satisfying (3.41). Thus

$$
T_{5} V(s)=0 .
$$


Now consider the last block row of (3.42)

$$
\left[\begin{array}{lll}
R_{31} & E_{32} & E_{33}
\end{array}\right] V(s)=\left[\begin{array}{lll}
0 & 0 & I
\end{array}\right] U(-s)
$$

and substitute for $E_{33}$ as

$$
E_{33}=-\left(R_{31} R_{11}^{\sim}+E_{32} R_{12}^{\sim}\right)\left(R_{13}^{-1}\right)^{\sim}
$$

yielding

$$
R_{31}\left[\begin{array}{lll}
I & 0 & -R_{12}^{\sim}\left(R_{13}^{-1}\right)^{\sim}
\end{array}\right] V(s)+E_{32}\left[\begin{array}{lll}
0 & I & -R_{12}^{\sim}\left(R_{13}^{-1}\right)
\end{array}\right] V(s)=\left[\begin{array}{lll}
0 & 0 & I
\end{array}\right] U(-s) .
$$

The second term is $E_{32} T_{5} V(s)=0$ by (3.43). Hence

$$
\left[\begin{array}{lll}
I & 0 & -R_{11}^{\sim}\left(R_{13}^{-1}\right)^{\sim}
\end{array}\right] V(s)=R_{31}^{-1}\left[\begin{array}{lll}
0 & 0 & I
\end{array}\right] U(-s) .
$$

The left-hand side of (3.44) is in $\mathscr{R} \mathscr{H}_{2}$ where the right-hand side is in $\mathscr{R} \mathscr{H} \frac{\perp}{2}$ and hence both must be zero. This together with (3.28) and (3.43) gives that $T_{2} V(s)=0$ which, when substituted into (3.42) using (3.26), gives

$$
U(-s)=T_{3} V(s)
$$

but $U(-s) \in \mathscr{R} \mathscr{H}_{2}^{\perp}$ and $T_{3} V(s) \in \mathscr{R} \mathscr{H}_{2}$ so that both must be zero contradicting $U$ being the Laplace transform of a Schmidt vector.

3.2. State-space construction and sufficient conditions. We will first construct a state-space description of $R_{\mathrm{a}}$ given in (3.32). The terms $R_{13}$ and $R_{31}$ come from standard spectral factorization problems and it is a routine exercise to find out the realization of $R_{a}$.

LEMMA 3.4. Let

$$
\left[\begin{array}{ll}
R_{11} & R_{12} \\
R_{21} & R_{22}
\end{array}\right] \in \mathscr{R} \mathscr{H}_{\infty}^{-}
$$

be such that (3.2) holds for some $Q_{22} \in \mathscr{R} \mathscr{L}_{\infty}$ and have the state-space realization

$$
\left[\begin{array}{ll}
R_{11} & R_{12} \\
R_{21} & R_{22}
\end{array}\right] \stackrel{s}{=}\left[\begin{array}{c|cc}
A & B_{1} & B_{2} \\
\hline C_{1} & D_{11} & D_{12} \\
C_{2} & D_{21} & 0
\end{array}\right]
$$

where $\operatorname{Re} \lambda_{i}(A)>0$ for all $i$. Then $R_{a}$ given by (3.32) has a state-space realization of the form

$$
R_{a} \stackrel{s}{=}\left[\begin{array}{c|ccc}
A & B_{1} & B_{2} & B_{3} \\
\hline C_{1} & D_{11} & D_{12} & D_{13} \\
C_{2} & D_{21} & * & * \\
C_{3} & D_{31} & * & *
\end{array}\right]
$$

where

$$
\begin{aligned}
& D_{13} D_{13}^{\prime}=I-D_{11} D_{11}^{\prime}-D_{12} D_{12}^{\prime}>0 \text {, } \\
& D_{31}^{\prime} D_{31}=I-D_{11}^{\prime} D_{11}-D_{21}^{\prime} D_{21}>0 \text {, } \\
& B_{3}=\left(X C_{1}^{\prime}-\left[\begin{array}{ll}
B_{1} & B_{2}
\end{array}\right]\left[\begin{array}{ll}
D_{11} & D_{12}
\end{array}\right]^{\prime}\right)\left(D_{13}^{-1}\right)^{\prime} \text {, } \\
& C_{3}=\left(D_{31}^{-1}\right)^{\prime}\left(B_{1}^{\prime} Y-\left[\begin{array}{ll}
D_{11}^{\prime} & D_{21}^{\prime}
\end{array}\right]\left[\begin{array}{ll}
C_{1}^{\prime} & C_{2}^{\prime}
\end{array}\right]^{\prime}\right) \text {. }
\end{aligned}
$$

$X=X^{\prime} \geqq 0$ is the unique solution to the algebraic Riccati equation

$$
-X A^{\prime}-A X+\left[\begin{array}{ll}
B_{1} & B_{2}
\end{array}\right]\left[\begin{array}{ll}
B_{1} & B_{2}
\end{array}\right]^{\prime}+B_{3} B_{3}^{\prime}=0
$$


such that $\operatorname{Re} \lambda_{i}\left(A-B_{3} D_{13}^{-1} C_{1}\right) \geqq 0$ for all $i . \quad Y=Y^{\prime} \geqq 0$ is the unique solution to the algebraic Riccati equation

$$
-Y A-A^{\prime} Y+\left[\begin{array}{ll}
C_{1}^{\prime} & C_{2}^{\prime}
\end{array}\right]\left[\begin{array}{ll}
C_{1}^{\prime} & C_{2}^{\prime}
\end{array}\right]^{\prime}+C_{3}^{\prime} C_{3}=0
$$

such that $\operatorname{Re} \lambda_{i}\left(A-B_{1} D_{31}^{-1} C_{3}\right) \geqq 0$ for all $i$.

Proof. The construction of $B_{3}$ and $D_{13}$ to form $R_{13}$, and $C_{3}$ and $D_{31}$ to form $R_{31}$ uses standard techniques for calculating spectral factors using Riccati equations [1], [50].

The realization of $R_{a}$ clearly matches $R_{11}, R_{12}, R_{13}, R_{21}, R_{22}$, and $R_{31}$. It remains to verify that $R_{23}, R_{32}$, and $R_{33}$ given in (3.32) match the given realization. This is a routine state-space manipulation and uses the following realizations:

$$
\left(R_{31}^{-1}\right)^{\sim}\left[\begin{array}{ll}
R_{11}^{\sim} & R_{21}^{\sim}
\end{array}\right] \stackrel{s}{=}\left[\begin{array}{c|c}
-A^{\prime}+C_{3}^{\prime}\left(D_{31}^{-1}\right)^{\prime} B_{1}^{\prime} & C_{3}^{\prime}\left(D_{31}^{-1}\right)^{\prime}\left[\begin{array}{cc}
D_{11}^{\prime} & D_{21}^{\prime}
\end{array}\right]-\left[\begin{array}{ll}
C_{1}^{\prime} & C_{2}^{\prime}
\end{array}\right] \\
\hline\left(D_{31}^{-1}\right)^{\prime} B_{1}^{\prime} & \left(D_{31}^{-1}\right)^{\prime}\left[\begin{array}{ll}
D_{11}^{\prime} & D_{21}^{\prime}
\end{array}\right]
\end{array}\right]
$$

and the dual

$$
\left[\begin{array}{c}
R_{11}^{\sim} \\
R_{12}^{\sim}
\end{array}\right]\left(R_{13}^{-1}\right)^{\sim} \stackrel{s}{=}\left[\begin{array}{c|c}
-A^{\prime}+C_{1}^{\prime}\left(D_{13}^{-1}\right)^{\prime} B_{3}^{\prime} & C_{1}^{\prime}\left(D_{13}^{-1}\right)^{\prime} \\
\hline\left[\begin{array}{c}
D_{11}^{\prime} \\
D_{12}^{\prime}
\end{array}\right]\left(D_{13}^{-1}\right)^{\prime} B_{3}^{\prime}-\left[\begin{array}{l}
B_{1}^{\prime} \\
B_{2}^{\prime}
\end{array}\right] & {\left[\begin{array}{l}
D_{11}^{\prime} \\
D_{12}^{\prime}
\end{array}\right]\left(D_{13}^{-1}\right)^{\prime}}
\end{array}\right] .
$$

Equations (3.50) and (3.52) then give that

$\left(R_{31}^{-1}\right)^{\sim}\left[\begin{array}{ll}R_{11}^{\sim} & R_{21}^{\sim}\end{array}\right]\left[\begin{array}{l}C_{1} \\ C_{2}\end{array}\right](s I-A)^{-1}=-C_{3}(s I-A)^{-1}+\{$ terms in analytic in $\operatorname{Re}(s)>0\}$ and (3.49) and (3.51) imply that

$$
(s I-A)^{-1}\left[\begin{array}{ll}
B_{1} & B_{2}
\end{array}\right]\left[\begin{array}{l}
R_{11}^{\sim} \\
R_{21}^{\sim}
\end{array}\right]\left(R_{13}^{-1}\right)^{\sim}=-(s I-A)^{-1} B_{3}+\{\text { terms in analytic in } \operatorname{Re}(s)>0\} .
$$

The definition of $\boldsymbol{R}_{a}$ in (3.32) together with (3.53) and (3.54) then give the result.

We now immediately have the following corollaries of Propositions 3.2 and 3.3, on noting that $X$ and $Y$ are the Gramians for $R_{a}$ and hence $\left\|R_{a}\right\|_{H}^{2}=\rho(X Y)$ [14], [6].

Corollary 3.5. Let

$$
\left[\begin{array}{ll}
R_{11} & R_{12} \\
R_{21} & R_{22}
\end{array}\right] \in \mathscr{R} \mathscr{H}_{\infty}^{-}
$$

satisfy (3.3) and (3.4) with the state-space realization of (3.45). Then

(i) If there exists $Q_{22} \in \mathscr{R} \mathscr{H}_{\infty}^{+}$satisfying (3.2), then $\rho(X Y) \leqq 1$.

(ii) If there exists $Q_{22} \in \mathscr{R} \mathscr{H}_{\infty}^{+}$satisfying (3.1), then $\rho(X Y)<1$.

Now that we have an explicit state-space realization of $R_{a}$ and a condition on the Gramians we can attempt a state-space constrution of an all-pass embedding along the lines of (3.3) (without assuming knowledge of a candidate $Q_{22}$ ). This construction can be carried out along the lines of Glover [17], [18] and will then give a sufficiency proof. We will consider the case $\left\|R_{a}\right\|_{H}<1$ in this section with the $\left\|R_{a}\right\|_{H}=1$ considered in $\S 4$. 
First, consider the unitary dilation of the $D$-matrix. Such a dilation can be constructed in the form

$$
D_{e}=\begin{array}{cccc}
m_{1} & m_{2} & m_{3}=p_{1} & m_{4} \leqq p_{2} \\
p_{1} \\
p_{2} \\
p_{3}=m_{1} \\
m_{2} \geqq p_{4}
\end{array}\left[\begin{array}{cccc}
D_{11} & D_{12} & D_{13} & 0 \\
D_{21} & D_{22} & D_{23} & D_{24} \\
D_{31} & D_{32} & D_{33} & D_{34} \\
0 & D_{42} & D_{43} & 0
\end{array}\right] .
$$

In (3.55) $\left[\begin{array}{l}D_{24} \\ D_{34}\end{array}\right]$ is chosen to be an orthonormal basis for the nullspace of $\left[\begin{array}{ll}D_{21}^{\prime} & D_{31}^{\prime}\end{array}\right]$ (which has dimension $p_{2}$ since rank $D_{31}=m_{1}$, and also implies that $D_{24}^{-1}$ exists). Similarly, $\left[\begin{array}{ll}D_{42} & D_{43}\end{array}\right]^{\prime}$ is an orthonormal basis for the nullspace of $\left[\begin{array}{ll}D_{12} & D_{13}\end{array}\right]$, and $D_{42}^{-1}$ exists. The remaining terms are then uniquely given by

$$
\left[\begin{array}{ll}
D_{22} & D_{23} \\
D_{32} & D_{33}
\end{array}\right]=-\left[\begin{array}{cc}
D_{21}^{\prime} & D_{31}^{\prime} \\
D_{24}^{\prime} & D_{34}^{\prime}
\end{array}\right]^{-1}\left[\begin{array}{cc}
D_{11}^{\prime} & 0 \\
0 & 0
\end{array}\right]\left[\begin{array}{cc}
D_{12} & D_{13} \\
D_{42} & D_{43}
\end{array}\right] .
$$

Now suppose that we can find a realization of $E_{a a}=R_{a a}+Q_{a a}$ in the form

$$
\begin{gathered}
R_{a a} \stackrel{s}{=}\left[\begin{array}{c|c}
A & B_{e} \\
\hline C_{e} & *
\end{array}\right] \stackrel{s}{=}\left[\begin{array}{cc}
R_{a} & 0 \\
0 & 0
\end{array}\right], \\
Q_{a a} \stackrel{s}{=}\left[\begin{array}{c|c}
\hat{A} & \hat{B}_{e} \\
\hline \hat{C}_{e} & 0
\end{array}\right], \quad \operatorname{Re} \lambda_{i}(\hat{A})<0 \quad \forall i
\end{gathered}
$$

where

$$
\begin{aligned}
& B_{e}=\left[\begin{array}{llll}
B_{1} & B_{2} & B_{3} & 0
\end{array}\right], \\
& C_{e}^{\prime}=\left[\begin{array}{llll}
C_{1}^{\prime} & C_{2}^{\prime} & C_{3}^{\prime} & 0
\end{array}\right], \\
& \hat{B}_{e}=\left[\begin{array}{llll}
0 & \hat{B}_{2} & \hat{B}_{3} & \hat{B}_{4}
\end{array}\right], \\
& \hat{C}_{e}^{\prime}=\left[\begin{array}{llll}
0 & \hat{C}_{2}^{\prime} & \hat{C}_{3}^{\prime} & \hat{C}_{4}^{\prime}
\end{array}\right],
\end{aligned}
$$

and

$$
E_{a a} \stackrel{s}{=}\left[\begin{array}{cc|c}
A & 0 & B_{e} \\
0 & \hat{A} & \hat{B}_{e} \\
\hline C_{e} & \hat{C}_{e} & D_{e}
\end{array}\right]
$$

In order to construct $\hat{A}, \hat{B}_{e}$, and $\hat{C}_{e}$ such that $E_{a a}$ is all-pass, we will write the all-pass lemma equations of Glover [16, Thm. 5.1] with postulated solutions to the Lyapunov equations as follows:

$$
X_{e}=\left[\begin{array}{cc}
-X & I \\
I & Y Z^{-1}
\end{array}\right]
$$

where

$$
\begin{gathered}
Z=I-X Y \\
Y_{e}=X_{e}^{-1}=\left[\begin{array}{cc}
-Y & Z^{\prime} \\
Z & Z X
\end{array}\right] .
\end{gathered}
$$


(The form of $X_{e}$ and $Y_{e}$ essentially comes from Glover [16, Lemma 8.2] but this need not concern us.) With this value for $X_{e}, \hat{A}, \hat{B}_{e}, \hat{C}_{e}$ can be constructed to satisfy the conditions of $[16, T h m .5 .1]$, viz. $D_{e}$ unitary and

$$
\begin{gathered}
{\left[\begin{array}{cc}
A & 0 \\
0 & \hat{A}
\end{array}\right] X_{e}+X_{e}\left[\begin{array}{cc}
A^{\prime} & 0 \\
0 & \hat{A}^{\prime}
\end{array}\right]+\left[\begin{array}{l}
B_{e} \\
\hat{B}_{e}
\end{array}\right]\left[\begin{array}{ll}
B_{e}^{\prime} & \hat{B}_{e}^{\prime}
\end{array}\right]=0,} \\
D_{e}\left[\begin{array}{ll}
B_{e}^{\prime} & \hat{B}_{e}^{\prime}
\end{array}\right]+\left[\begin{array}{ll}
C_{e} & \hat{C}_{e}
\end{array}\right] X_{e}=0 .
\end{gathered}
$$

Postmutiplying (3.68) by the invertible matrix $\left[\begin{array}{cc}I & -Y \\ 0 & Z\end{array}\right]$ yields the equivalent expressions

$$
\hat{C}_{e}=C_{e} X-D_{e} B_{e}^{\prime}
$$

and

$$
-D_{e} B_{e}^{\prime} Y+D_{e} \hat{B}_{e}^{\prime} Z+C_{e}=0
$$

Since $Z$ is nonsingular

$$
\hat{B}_{e}=\left(Z^{-1}\right)^{\prime}\left(Y B_{e}-C_{e}^{\prime} D_{e}\right)
$$

and (3.68) holds.

Now let $E$ be the left-hand side of (3.67); then $\left[\begin{array}{ll}I & 0\end{array}\right] E\left[\begin{array}{ll}I & 0\end{array}\right]^{\prime}=0$ by (3.51) and $\left[\begin{array}{ll}I & 0\end{array}\right] Y_{e} E Y_{e}\left[\begin{array}{ll}I & 0\end{array}\right]^{\prime}=0$ by (3.52). $\hat{A}$ will be chosen to make $\left[\begin{array}{ll}I & 0\end{array}\right] E\left[\begin{array}{ll}0 & I\end{array}\right]^{\prime}=0$ as

$$
\begin{aligned}
\hat{A} & =-A^{\prime}-\hat{B}_{e} B_{e}^{\prime} \\
& =\left(Z^{-1}\right)^{\prime}\left\{-(I-Y X) A^{\prime}-\left(Y B_{e}-C_{e}^{\prime} D_{e}\right) B_{e}^{\prime}\right\} \\
& =\left(Z^{-1}\right)^{\prime}\left\{-A^{\prime}-Y A X+C_{e}^{\prime} D_{e} B_{e}^{\prime}\right\} .
\end{aligned}
$$

With this value of $\hat{A}$ we obtain $\left[\begin{array}{ll}I & 0\end{array}\right] E=0$ and hence

$$
\left[\begin{array}{cc}
I & 0 \\
-Y & Z^{\prime}
\end{array}\right] E\left[\begin{array}{cc}
I & -Y \\
0 & Z
\end{array}\right]=0
$$

and thus $E=0$. Therefore, with these values of $\hat{A}, \hat{B}_{e}$, and $\hat{C}_{e}, E_{a}$ satisfies (3.67) and (3.68) and is hence all-pass. Furthermore, $Y Z^{-1} \geqq 0$ since $\rho(X Y)<1$ by Corollary 3.5 and hence $\operatorname{Re} \lambda_{i}(\hat{A})<0$ (by Wonham [52, Lemma 12.2, p. 227]) since $\hat{A} Y Z^{-1}+$ $Z^{\prime-1} Y \hat{A}^{\prime}+\hat{B}_{e} \hat{B}_{e}^{\prime}=0$ and $\left(\hat{A}, \hat{B}_{e}\right)$ is clearly stabilizable. Therefore $Q_{a a} \in \mathscr{R} \mathscr{H}_{\infty}^{+}$and it remains to show that $\hat{B}_{e}$ and $\hat{C}_{e}$ have the zero terms given in (3.61) and (3.62). From (3.70) and (3.50)

$$
Z^{\prime} \hat{B}_{e}\left[\begin{array}{llll}
I & 0 & 0 & 0
\end{array}\right]^{\prime}=Y B_{1}-\left[\begin{array}{lll}
C_{1}^{\prime} & C_{2}^{\prime} & C_{3}^{\prime}
\end{array}\right]\left[\begin{array}{c}
D_{11} \\
D_{21} \\
D_{31}
\end{array}\right]=0 .
$$

Similarly, (3.69) and (3.49) give that

$$
\left[\begin{array}{llll}
I & 0 & 0 & 0
\end{array}\right] \hat{C}_{e}=C_{1} X-\left[\begin{array}{lll}
D_{11} & D_{12} & D_{13}
\end{array}\right]\left[\begin{array}{l}
B_{1}^{\prime} \\
B_{2}^{\prime} \\
B_{3}^{\prime}
\end{array}\right]=0 .
$$

Therefore we have verified that $Q_{a a}$ given by (3.58), (3.72), (3.69), and (3.70) satisfy (3.61) and (3.62) and, for all unitary $D_{e}$, gives an all-pass $E_{a a}$ with $Q_{a a} \in \mathscr{R} \mathscr{H}_{\infty}^{+}$. Note that once the form of $D_{e}$ and $X_{e}$ have been specified, all the other terms are uniquely determined and the required zero structure on $Q_{a a}$ has been ensured by fixing the form of the first row and column of $R_{a a}$ to be as in (3.5). All solutions can also be generated from this $Q_{a a}$ as will now be stated in the main result of this section. 
THEOREM 3.6. Let $R_{i j} \in \mathscr{R} \mathscr{H}_{\infty}^{-, p_{i} \times m_{j}}$ for $i, j=1,2$ have the realization

$$
\left[\begin{array}{cc}
R_{11} & R_{12} \\
R_{21} & R_{22}
\end{array}\right] \stackrel{s}{=}\left[\begin{array}{c|cc}
A & B_{1} & B_{2} \\
\hline C_{1} & D_{11} & D_{12} \\
C_{2} & D_{21} & *
\end{array}\right]
$$

with $\operatorname{Re} \lambda_{i}(A)>0$ for all $i$, and

$$
\bar{\sigma}\left[\begin{array}{ll}
D_{11} & D_{12}
\end{array}\right]<1, \quad \bar{\sigma}\left[\begin{array}{ll}
D_{11}^{\prime} & D_{21}^{\prime}
\end{array}\right]<1 .
$$

(a) Then there exists $Q \in \mathscr{R} \mathscr{H}_{\infty}^{+}$such that

$$
\left\|\left[\begin{array}{cc}
R_{11} & R_{12} \\
R_{21} & R_{22}+Q
\end{array}\right]\right\|_{\infty}<1
$$

if and only if (3.37), (3.38) and $\left\|R_{a}\right\|_{H}<1$, or equivalently if and only if (3.37), (3.38) and $\rho(X Y)<1$ where $X, Y \geqq 0$ are as defined in Lemma 3.4.

(b) If the conditions of part (a) are satisfied, then each $Q \in \mathscr{R} \mathscr{H}_{\infty}^{+}$satisfying (3.73) is given by

$$
Q=F_{l}\left\{\left[\begin{array}{ll}
Q_{22} & Q_{24} \\
Q_{42} & Q_{44}
\end{array}\right] ; \Phi\right\}
$$

for some $\Phi \in \mathscr{R} \mathscr{H}_{\infty}^{+}$and $\|\Phi\|_{\infty}<1$, where

$$
\left[\begin{array}{ll}
Q_{22} & Q_{24} \\
Q_{42} & Q_{44}
\end{array}\right] \stackrel{s}{=}\left[\begin{array}{c|cc}
\hat{A} & \hat{B}_{2} & \hat{B}_{4} \\
\hline \hat{C}_{2} & D_{22} & D_{24} \\
\hat{C}_{4} & D_{42} & 0
\end{array}\right]
$$

with $D_{i j}, D_{e}$ given by (3.55). The remaining matrices are given by

$$
\left[\begin{array}{c}
\hat{C}_{2} \\
\hat{C}_{4}
\end{array}\right]=\left[\begin{array}{c}
C_{2} X \\
0
\end{array}\right]-\left[\begin{array}{ccc}
D_{21} & D_{22} & D_{23} \\
0 & D_{42} & D_{43}
\end{array}\right]\left[\begin{array}{c}
B_{1}^{\prime} \\
B_{2}^{\prime} \\
B_{3}^{\prime}
\end{array}\right]
$$

$$
\left[\begin{array}{ll}
\hat{B}_{2} & \hat{B}_{4}
\end{array}\right]=\left(Z^{-1}\right)^{\prime}\left\{Y\left[\begin{array}{ll}
B_{2} & 0
\end{array}\right]-\left[\begin{array}{lll}
C_{1}^{\prime} & C_{2}^{\prime} & C_{3}^{\prime}
\end{array}\right]\right\}\left[\begin{array}{cc}
D_{12} & 0 \\
D_{22} & D_{24} \\
D_{32} & D_{34}
\end{array}\right]
$$

$$
Z=I-X Y, \quad \hat{A}=\left(Z^{-1}\right)^{\prime}\left\{-A^{\prime}-Y A X+C_{e}^{\prime} D_{e} B_{e}^{\prime}\right\} .
$$

(c) If $R_{i j}$ satisfies (3.34) and (3.35) and $\rho(X Y)<1$, then every solution $Q \in \mathscr{R} \mathscr{H}_{\infty}^{+}$ such that

$$
\left\|\left[\begin{array}{cc}
R_{11} & R_{12} \\
R_{21} & R_{22}+Q
\end{array}\right]\right\|_{\infty} \leqq 1
$$

is given by (3.74) for some $\Phi \in \mathscr{R} \mathscr{H}_{\infty}^{+}$with $\|\Phi\|_{\infty} \leqq 1$.

Proof. Part (a) follows from Propositions 3.2 and 3.3, Corollary 3.5, and the construction preceding the theorem statement. Part (b) follows in a similar way to that given in Glover [17], [18] as follows. First, recall that $E_{a a}$ is all-pass where

$$
E_{a a}(s)=\left[\begin{array}{cccc}
R_{11} & R_{12} & R_{13} & 0 \\
R_{21} & E_{22} & E_{23} & Q_{24} \\
R_{31} & E_{32} & E_{33} & Q_{34} \\
0 & Q_{42} & Q_{43} & Q_{44}
\end{array}\right]
$$


and hence,

$$
F_{l}\left\{E_{a a},\left[\begin{array}{cc}
0 & 0 \\
0 & \Phi
\end{array}\right]\right\}=\left[\begin{array}{cc}
R_{11} & R_{12} \\
R_{21} & R_{22}+Q
\end{array}\right] \text { satisfies }\left\|\left[\begin{array}{cc}
R_{11} & R_{12} \\
R_{21} & R_{22}+Q
\end{array}\right]\right\|_{\infty}<1
$$

if $\|\Phi\|_{\infty}<1$ [19], [42] where

$$
Q=F_{l}\left\{\left[\begin{array}{ll}
Q_{22} & Q_{24} \\
Q_{42} & Q_{44}
\end{array}\right] ; \Phi\right\} \in \mathscr{R} \mathscr{H}_{\infty}^{+} .
$$

That $Q$ is in $\mathscr{R} \mathscr{H}_{\infty}^{+}$follows from a small gain argument since $Q_{i j} \in \mathscr{R} \mathscr{H}_{\infty}^{+}$and $\Phi \in \mathscr{R} \mathscr{H}_{\infty}^{+}$ with $\left\|Q_{44} \Phi\right\|_{\infty}<1$. Hence all such $Q$ satisfy (3.73).

To show that all such $Q$ can be expressed as in (3.74) we first show that $Q_{24}^{-1}$, $Q_{42}^{-1} \in \mathscr{R} \mathscr{H}_{\infty}^{+}$. The $A$-matrix of $Q_{24}^{-1}$ is given by

$$
\begin{aligned}
\hat{A}-\hat{B}_{2} D_{42}^{-1} \hat{C}_{4} & =-A^{\prime}-\hat{B}_{e} B_{e}^{\prime}-\hat{B}_{2}\left(-B_{2}^{\prime}-D_{42}^{-1} D_{43} B_{3}^{\prime}\right) \\
& =-A^{\prime}-\left(\hat{B}_{3}-\hat{B}_{2} D_{42}^{-1} D_{43}\right) B_{3}^{\prime}
\end{aligned}
$$

since $D_{e}$ is unitary, $D_{42}^{-1} D_{43}=-D_{12}^{\prime}\left(D_{13}^{-1}\right)^{\prime}$; hence,

$$
\begin{aligned}
\hat{B}_{3}-\hat{B}_{2} D_{42}^{-1} D_{43} & =\left[\begin{array}{ll}
\hat{B}_{2} & \hat{B}_{3}
\end{array}\right]\left[\begin{array}{l}
D_{12}^{\prime} \\
D_{13}
\end{array}\right]\left(D_{13}^{-1}\right)^{\prime} \\
& =\hat{B}_{e} D_{e}^{\prime}\left[\begin{array}{l}
I \\
0 \\
0 \\
0
\end{array}\right]\left(D_{13}^{-1}\right)^{\prime} \text { by }(3.70) \\
& =\left(Z^{-1}\right)^{\prime}\left(Y B_{e} D_{e}^{\prime}\left[\begin{array}{l}
I \\
0 \\
0 \\
0
\end{array}\right]-C_{1}^{\prime}\right)\left(D_{13}^{-1}\right)^{\prime} \\
& =\left(Z^{-1}\right)^{\prime}(Y X-I) C_{1}^{\prime}\left(D_{13}^{-1}\right)^{\prime} \text { by }(3.49) \\
& =-C_{1}^{\prime}\left(D_{13}^{-1}\right)^{\prime} .
\end{aligned}
$$

Hence $\hat{A}-\hat{B}_{2} D_{42}^{-1} \hat{C}_{4}=-\left(A^{\prime}-C_{1}^{\prime}\left(D_{13}^{-1}\right)^{\prime} B_{3}^{\prime}\right) \quad$ and $\quad Q_{42}^{-1} \in \mathscr{R} \mathscr{H}_{\infty}^{+} \quad$ since $\operatorname{Re} \lambda_{i}(A-$ $\left.B_{3} D_{13}^{-1} C_{1}\right)<0$ are the zeros of $R_{13}$ which, by construction, has no zeros in $\operatorname{Re} s>0$. See (3.51).

A similar argument gives that $\hat{A}-\hat{B}_{4} D_{24}^{-1} \hat{C}_{2}=-\left(Z^{-1}\right)^{\prime}\left(A-B_{1} D_{31}^{-1} C_{3}\right)^{\prime} Z^{\prime}$ and hence $Q_{24}^{-1} \in \mathscr{R} \mathscr{H}_{\infty}^{+}$. Suppose that $Q$ satisfies (3.73) and define $\Phi$ such that (3.74) holds, that is,

$$
\Psi:=Q_{24}^{-1}\left(Q-Q_{22}\right) Q_{42}^{-1}=\Phi\left(I-Q_{44} \Phi\right)^{-1}
$$

and

$$
\Phi=-\left(I+\Psi Q_{44}\right)^{-1} \Psi
$$

and $\Phi$ clearly exists as a proper rational function in $\mathscr{L}_{\infty}$. Furthermore $\|\Phi\|_{\infty}<1$ since

$$
\| F_{l}\left(E_{a a},\left(\begin{array}{ll}
0 & 0 \\
0 & \Phi
\end{array}\right) \|_{\infty}<1, \quad Q_{44}(\infty)=0\right.
$$

(see [30]). Suppose that $\Phi$ has a coprime factorization over $\mathscr{R} \mathscr{H}_{\infty}^{+}$as $\Phi=U V^{-1}$ with $U, V \in \mathscr{R} \mathscr{H}_{\infty}^{+}$. Since $U, V$ is coprime and $Q_{44}$ is stable, $U,\left(V-Q_{44} U\right)$ is also coprime, 
and a coprime factorization of $\Psi=U\left(V-Q_{44} U\right)^{-1}$. Since $\Psi \in \mathscr{R}_{\infty}^{+}$the winding number of $\operatorname{det}\left(V-Q_{44} U\right)(j \omega)$ around the origin must be zero. However, det $(V-$ $\left.Q_{44} U\right)(j \omega)=\operatorname{det} V(j \omega) \operatorname{det}\left(I-Q_{44} \Phi\right)(j \omega)$ and hence the winding number of det $V(j \omega)$ is zero, since that of $\operatorname{det}\left(I-Q_{44} \Phi\right)(j \omega)$ is zero (because $\left.\left\|Q_{44} \Phi\right\|_{\infty}<1\right)$ hence $\Phi \in \mathscr{R} \mathscr{H}_{\infty}^{+}$.

(c) The major difference between this case and part (b) is that $R_{13}^{-1}, R_{31}^{-1}$ need not be in $\mathscr{R} \mathscr{H}_{\infty}^{-}$and $Q_{24}^{-1}, Q_{42}^{-1}$ need not be in $\mathscr{R} \mathscr{H}_{\infty}^{+}$; however, $R_{13}, R_{31}, Q_{24}$, and $Q_{42}$ will still be full rank in the appropriate halfplanes and only rank deficient at a finite number of points on $s=j \omega$. The proof that (3.74) with $\|\Phi\|_{\infty} \leqq 1$ gives a class of solutions for $Q$ is the same as in part (b) except that we now need to prove that $\left\|Q_{44}\right\|_{\infty}<1$. Suppose $\left\|Q_{44}\right\|_{\infty}=1$. Since $E_{a a}$ is all-pass if $\bar{\sigma}\left(Q_{44}\left(j \omega_{0}\right)\right)=1$ for some $\omega_{0}$ then $\operatorname{rank}\left[Q_{42}\left(j \omega_{0}\right) \quad Q_{43}\left(j \omega_{0}\right)\right]<p_{4}=m_{3}=p_{1}$, i.e., $\left[\begin{array}{ll}Q_{42} & Q_{43}\end{array}\right]$ has an imaginary axis zero. Now

$$
\begin{aligned}
{\left[\begin{array}{ll}
Q_{42} & Q_{43}
\end{array}\right] } & \stackrel{s}{=}\left[\begin{array}{c|cc}
\hat{A} & \hat{B}_{2} & \hat{B}_{3} \\
\hline \hat{C}_{4} & D_{42} & D_{43}
\end{array}\right] \\
& =\left[\begin{array}{ll}
D_{42} & D_{43}
\end{array}\right]\left[\begin{array}{c|cc}
\hat{A} & \hat{B}_{2} & \hat{B}_{3} \\
\hline-B_{2}^{\prime} & I & 0 \\
-B_{3}^{\prime} & 0 & I
\end{array}\right] \text { from (3.76) }
\end{aligned}
$$

and

$$
\hat{A}+\left[\begin{array}{ll}
\hat{B}_{2} & \hat{B}_{3}
\end{array}\right]\left[\begin{array}{ll}
B_{2} & B_{3}
\end{array}\right]^{\prime}=-A^{\prime} \quad \text { by }(3.71)
$$

and hence the zeros of $\left[\begin{array}{ll}Q_{42} & Q_{43}\end{array}\right]$ can only appear at $\lambda_{i}\left(-A^{\prime}\right)$. But $\operatorname{Re} \lambda_{i}\left(-A^{\prime}\right)<0$. Thus rank $\left[Q_{42}(j \omega) Q_{43}(j \omega)\right]=p_{4}=m_{3}=p_{1}$ for all $\omega$, giving $\left\|Q_{44}\right\|_{\infty}<1$.

To prove that (3.74) with $\|\Phi\|_{\infty} \leqq 1$ gives all solutions we first need that

$$
\| F_{l}\left(E_{a a},\left(\begin{array}{ll}
0 & 0 \\
0 & \Phi
\end{array}\right) \|_{\infty} \leqq 1\right.
$$

implies $\|\Phi\|_{\infty} \leqq 1$, when $R_{13}, R_{31}, Q_{24}, Q_{42}$ are only full rank for almost all $s=j \omega$. This is simply proved by noting that if $\bar{\sigma}\left(\Phi\left(j \omega_{0}\right)\right)>1$ then $\bar{\sigma}(\Phi(s))>1$ for all $s$ in some neighbourhood of $j \omega_{0}$, and hence we can find $\omega_{1}$ such that $\bar{\sigma}\left(\Phi\left(j \omega_{1}\right)\right)>1$ and $R_{13}$, $R_{31}, E_{24}$, and $E_{42}$ all have full rank at $j \omega_{1}$; a contradiction is then easily established.

Finally, defining $\Psi$ and $\Phi$ as in (b) with $\Phi=U V^{-1}$ we obtain that $\operatorname{det} V(j \omega) \neq 0$ for all $\omega$ since $\|\Phi\|_{\infty} \leqq 1, \operatorname{det}\left(I-Q_{44} \Phi\right)(j \omega) \neq 0$ for all $\omega$ since $\left\|Q_{44} \Phi\right\|_{\infty}<1$, and hence $\Psi=U\left(V-Q_{44} U\right)^{-1}$ and $\operatorname{det}\left(V-Q_{44} U\right)=\operatorname{det}(V) \operatorname{det}\left(I-Q_{44} \Phi\right) \neq 0$ for all $\omega$ implies that $\Psi \in \mathscr{R} \mathscr{H}_{\infty}$ and the result follows as in (b).

Remark. Theorem 3.6 gives a complete solution to the problem when $\rho(X Y)<1$, the case when $\rho(X Y)=1$ is substantially more involved and is given in $\S 4$. The solution of

$$
\left\|\left[\begin{array}{cc}
R_{11} & R_{12} \\
R_{21} & R_{22}+Q
\end{array}\right]\right\|_{\infty} \leqq \gamma
$$

for the minimum possible $\gamma$ will require an iterative search on $\gamma$ with the problem scaled to $\gamma$ at each step (e.g., scale $D_{i j} \rightarrow \gamma^{-1} D_{i j}, B_{i} \rightarrow \gamma^{-1 / 2} B_{i}, C_{i} \rightarrow \gamma^{-1 / 2} C_{i}$ ). A value of $\gamma$ will be achievable if the algebraic Riccati equations for $X(\gamma)$ and $Y(\gamma)$ have solutions with $\rho(X(\gamma) Y(\gamma)) \leqq 1$. The optimal value of $\gamma$ can occur when $\rho(X(\gamma) Y(\gamma))=1$ or when $\gamma$ is the largest value such that the Hamiltonians for $X(\gamma)$ or $Y(\gamma)$ have imaginary axis eigenvalues. In this way the optimal value of $\gamma$ can be calculated to any desired accuracy and a problem with $\rho(X Y)=1$ normally results. 
4. Descriptor all-pass systems and the sufficient conditions. In this section we propose to lift the assumption that $\left\|R_{a}^{\sim}\right\|_{H}<1$ and treat the case when $\left\|R_{a}^{\sim}\right\|_{H}=1$. Before starting the general analysis, we consider a simple example which illustrates an important feature of the four block problem at optimality. Consider

$$
\inf _{q \in \mathscr{H}_{\infty}^{+}}\left\|\left[\begin{array}{cc}
r_{11} & 0 \\
0 & r_{22}+q
\end{array}\right]\right\|_{\infty}
$$

in which $r_{11}, r_{22} \in \mathscr{H}_{\infty}^{-}$. It is immediate that there are two cases which require separate consideration. These are

(1) $\left\|r_{22}\right\|_{H}<\left\|r_{11}\right\|_{\infty}$,

(2) $\left\|r_{22}\right\|_{H} \geqq\left\|r_{11}\right\|_{\infty}$.

In the first case, any $q(s) \in \mathscr{H}_{\infty}^{+}$for which $\left\|r_{22}+q\right\|_{\infty} \leqq\left\|r_{11}\right\|_{\infty}$ will be an optimal solution; a continuum of such solutions exist. In the second case, however, the (unique) optimal Nehari extension of $r_{22}(s)$ is the only solution. This example shows that there may or may not be a reduction in the size of the solution set at optimality. In the one block case, the size of the solution set always decreases at optimality [16].

In the general case there are also two forms of optimality. To see this we temporarily suppose that $Q(s)$ in (3.2) is allowed to range over $\mathscr{L}_{\infty}$. Under these conditions we have

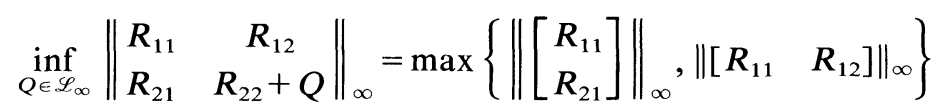

by Parrott's theorem [39], [41, Thm. 1.2]; a particularly nice treatment of Parrott's theorem is given in Young [53]. The point is that in certain cases

$$
\inf _{Q \in \mathscr{L}_{\infty}}\left\|\begin{array}{cc}
R_{11} & R_{12} \\
R_{21} & R_{22}+Q
\end{array}\right\|_{\infty}=\inf _{Q \in \mathscr{H}_{\infty}^{+}} \| \begin{array}{cc}
R_{11} & R_{12} \\
R_{21} & R_{22}+Q \|_{\infty}
\end{array}
$$

and the requirement that $Q$ be an element of $\mathscr{H}_{\infty}^{+}$rather than just $\mathscr{L}_{\infty}$ makes no difference to the achievable norm. This Parrott type of optimality has already been covered by Theorem 3.6. The alternative form of optimality is treated below.

4.1. All optimal solutions: the general case. The purpose of this section is to treat sufficiency in the case that $\left\|R_{a}^{\sim}\right\|_{H}=1$. We will take Lemma 3.4 as our starting point and then show that an all-pass embedding may still be constructed along the lines of Theorem 3.6. The key difficulty in the case of $\left\|R_{a}^{\sim}\right\|_{H}=1$ is that $Z$ in (3.65) is singular. Our approach to this problem will be to construct the all-pass embedding in a descriptor framework. We begin with an all-pass lemma for descriptor systems of the form (4.5) and (4.6) below. Apart from dealing with the standard case of a possibly singular $E$, we need to cater to the case of $\operatorname{det}(s E-A) \equiv 0 ;(s E-A)$ singular for all values of $s$.

THEOREM 4.1. Consider the descriptor system of equations

$$
\begin{gathered}
s \operatorname{Ex}(s)=A x(s)+B u(s), \\
y=C x(s)+D u(s),
\end{gathered}
$$

and suppose that there exists a matrix $T$ such that

$$
\begin{gathered}
A T+T^{\prime} A^{\prime}+B B^{\prime}=0, \\
E T=T^{\prime} E^{\prime} .
\end{gathered}
$$


(a) If $T$ is nonsingular and

$$
C T=L B^{\prime}
$$

for some L, (4.5) and (4.6) define a unique tranfer function given by

$$
G(s)=D+C(s E-A)^{\#} B
$$

in which $(\cdot)^{\#}$ denotes a generalized inverse (which is defined in (4.16) below).

(b) If $T$ is nonsingular and

(i) $D D^{\prime}=I$,

$$
\text { (ii) } C T+D B^{\prime}=0 \text {, }
$$

then

$$
G G^{\sim}=I
$$

(c) If $E T \geqq 0$ and $(s E-A, B)$ is stabilizable (i.e., $x^{\prime}(s E-A)=0, x^{\prime} E \neq 0, x^{\prime} B=0 \Rightarrow$ $(s+\bar{s})<0)$, then all the finite eigenvalues of $s E-A$ satisfy $(s+\bar{s})<0$.

Proof. In the proof of part (a), we use (4.7) and (4.8) to establish that $\mathscr{R}(B) \subset$ $\mathscr{R}(s E-A)$. This allows $(4.5)$ to be solved for $x(s)$ given any $u(s)$. Following that, (4.9) is used to prove that $\mathcal{N}(s E-A) \subset \mathcal{N}(C)$ which establishes the existence of the unique transfer function (4.10).

Suppose $s E-A$ has Smith diagonalization

$$
s E-A=N(s) F(s) M(s)
$$

in which $N(s)$ and $M(s)$ are unimodular polynomial matrices and

$$
F(s)=\left[\begin{array}{cc}
F_{1}(s) & 0 \\
0 & 0
\end{array}\right]
$$

We define

$$
(s E-A)^{\# \#}:=M^{-1}(s)\left[\begin{array}{cc}
F_{1}^{-1}(s) & 0 \\
0 & 0
\end{array}\right] N^{-1}(s) .
$$

To show that (4.5) has a solution for $x(s)$ given any $u(s)$, we note that (4.7) and (4.8) give

$$
(s E-A) T+T^{\prime}\left(-s E^{\prime}-A^{\prime}\right)=B B^{\prime}
$$

and hence from (4.14)

$$
\begin{aligned}
& F M T\left(N^{\sim}\right)^{-1}+N^{-1} T^{\prime} M^{\sim} F^{\sim}=N^{-1} B B^{\prime}\left(N^{\sim}\right)^{-1} \\
& \Rightarrow\left[\begin{array}{ll}
0 & I
\end{array}\right] N^{-1} B=0 .
\end{aligned}
$$

It is clear from (4.18) that $\mathscr{R}(B) \subset \mathscr{R}(s E-A)$ and thus that (4.5) has a solution for all $u(s)$. A simple verification shows that $x(s)$ solves (4.5) if and only if

$$
\begin{aligned}
x(s) & =M^{-1}(s)\left\{\left[\begin{array}{cc}
F_{1}^{-1} & 0 \\
0 & 0
\end{array}\right] N^{-1}(s) B u(s)+\left[\begin{array}{l}
0 \\
I
\end{array}\right] w(s)\right\} \\
& =(s E-A)^{\#} B u(s)+M^{-1}(s)\left[\begin{array}{l}
0 \\
I
\end{array}\right] w(s)
\end{aligned}
$$


for some $w(s)$. Next, we note that (4.17) gives

$$
\begin{aligned}
& \left(T^{\prime}\right)^{-1}(s E-A)+\left(-s E^{\prime}-A\right) T^{-1}=\left(T^{\prime}\right)^{-1} B B^{\prime} T^{-1} \\
& \Rightarrow\left(M^{\sim}\right)^{-1}\left(T^{\prime}\right)^{-1} N F+F^{\sim} N^{\sim} T^{-1} M^{-1}=\left(M^{\sim}\right)^{-1}\left(T^{\prime}\right)^{-1} B B^{\prime} T^{-1} M^{-1} \\
& \Rightarrow B^{\prime} T^{-1} M^{-1}\left[\begin{array}{l}
0 \\
I
\end{array}\right]=0 .
\end{aligned}
$$

Thus

$$
\begin{aligned}
y(s) & =C x(s)+D u(s) \\
& =D u(s)+C(s E-A)^{\#} B u(s)+C M^{-1}\left[\begin{array}{l}
0 \\
I
\end{array}\right] w(s) \\
& =\left\{D+C(s E-A)^{\#} B\right\} u(s)+L B^{\prime} T^{-1} M^{-1}\left[\begin{array}{l}
0 \\
I
\end{array}\right] w(s) \\
& =G(s) u(s)
\end{aligned}
$$

by (4.9) and (4.20).

(b) Equations (4.12), (4.14), and (4.16) imply that

$$
\begin{aligned}
C(s E-A)^{\#}(s E-A) & =-D B^{\prime} T^{-1} M^{-1}\left[\begin{array}{ll}
I & 0 \\
0 & 0
\end{array}\right] M \\
& =-D B^{\prime} T^{-1} \text { by }(4.20) \\
& =C \text { by }(4.12) .
\end{aligned}
$$

Thus

$$
\begin{aligned}
I-G G^{\sim}= & I-D D^{\prime}-C(s E-A)^{\#} B D^{\prime}-D B^{\prime}\left(-s E-A^{\prime}\right)^{\#} C^{\prime} \\
& -C(s E-A)^{\#}\left((s E-A) T+T^{\prime}\left(-s E^{\prime}-A^{\prime}\right)\right) \\
& \times\left(-s E-A^{\prime}\right)^{\#} C^{\prime} \quad \text { by }(4.10) \text { and }(4.17) \\
=0 & \text { by }(4.11),(4.12), \text { and }(4.21) .
\end{aligned}
$$

(c) Let $\lambda$ be a finite eigenvalue of $(s E-A)$. Then there exists a corresponding eigenvector $x$ such that (i) $x^{\prime} E \neq 0$ and $x^{\prime}(\lambda E-A)=0 . x^{\prime}(4.7) x \Rightarrow(\lambda+\bar{\lambda}) x^{\prime} E T x=$ $-x^{\prime} B B^{\prime} x \leqq 0$. If $x^{\prime} B \neq 0$, then $\lambda+\bar{\lambda}<0$ since $E T \geqq 0$. If $x^{\prime} B=0$, then $\lambda+\bar{\lambda}<0$ by the stabilizability assumption.

Proceeding as before, we postulate matrices $T$ and $E$ which are associated with equations (4.7) and (4.8), and which satisfy (4.12)

$$
T=\left[\begin{array}{cc}
-X & Z \\
I & Y
\end{array}\right], \quad E=\left[\begin{array}{cc}
I & 0 \\
0 & Z^{\prime}
\end{array}\right]
$$

where $X, Y$, and $Z$ are defined in (3.51), (3.52), and (3.65). Equations (3.67) and (3.68) become

$$
\left[\begin{array}{cc}
A & 0 \\
0 & \hat{A}_{0}
\end{array}\right]\left[\begin{array}{cc}
-X & Z \\
I & Y
\end{array}\right]+\left[\begin{array}{cc}
-X & I \\
Z^{\prime} & Y
\end{array}\right]\left[\begin{array}{cc}
A^{\prime} & 0 \\
0 & \hat{A}_{0}^{\prime}
\end{array}\right]+\left[\begin{array}{c}
B_{e} \\
\hat{B}_{e 0}
\end{array}\right]\left[\begin{array}{ll}
B_{e}^{\prime} & \hat{B}_{e 0}^{\prime}
\end{array}\right]=0
$$

and

$$
D_{e}\left[\begin{array}{ll}
B_{e}^{\prime} & \hat{B}_{e 0}^{\prime}
\end{array}\right]+\left[\begin{array}{ll}
C_{e} & \hat{C}_{e 0}
\end{array}\right]\left[\begin{array}{cc}
-X & Z \\
I & Y
\end{array}\right]=0
$$


respectively. Evaluating the first column of (4.25) and comparing with (3.69) gives

$$
\hat{C}_{e 0}=\hat{C}_{e}=C_{e} X-D_{e} B_{e}^{\prime} .
$$

In the same way, the second column of (4.25) together with (3.70) yields

$$
\hat{B}_{e 0}=Y B_{e}-C_{e}^{\prime} D_{e}=Z^{\prime} \hat{B}_{e}
$$

for any unitary $D_{e} . \hat{B}_{e 0}$ therefore retains the critical zero $(1,1)$ entry. The $(1,1)$ partition of $(4.24)$ is zero by $(3.51)$. From the $(2,1)$ partition of $(4.24)$ we obtain

$$
\hat{A}_{0}=-Z^{\prime} A^{\prime}-\hat{B}_{e 0} B_{e}^{\prime}=-A^{\prime}-Y A X+C_{e}^{\prime} D_{e} B_{e}^{\prime}=Z^{\prime} \hat{A} \text {. }
$$

It is now easy to check that the remaining equations in (4.24) are also satisfied.

We also note that $\left(Z^{\prime}, \hat{A}_{0}, \hat{B}_{e 0}\right)$ is stabilizable since if $x^{\prime} Z^{\prime} \neq 0, x^{\prime}\left[s Z^{\prime}-\hat{A}_{0}, \hat{B}_{e 0}\right]=$ $0 \Rightarrow x^{\prime}\left[s Z^{\prime}+Z^{\prime} A^{\prime}\right]=0 \Rightarrow s+\bar{s}<0$ since $\operatorname{Re}\left(\lambda_{i}(A)\right)>0$. We can now prove the sufficiency of the condition in Corollary 3.5(i).

THEOREM 4.2. Given that the conditions of Corollary 3.5(i) are satisfied, then

(a) $Q_{a a}(s):=D_{e}+\hat{C}_{e 0}\left(s Z^{\prime}-\hat{A}_{0}\right)^{\#} \hat{B}_{e 0} \in \mathscr{R}^{\infty}$,

(b) $R_{a a}+Q_{a a}$ is all-pass.

Hence the conditions are both necessary and sufficient.

Proof. Equations (4.24) and (4.25) give that Theorem 4.1 can be applied to show that $R_{a a}+Q_{a a}$ is well defined and all-pass. Since $R_{a a}^{\sim} \in \mathscr{R}_{\infty}, Q_{a a}$ has no poles on $s=j \omega$ or at infinity. All the finite poles will be at eigenvalues of $\left(s Z^{\prime}-\hat{A}_{0}\right)$ which are in the open left halfplane by Theorem $4.1(\mathrm{c})$ since $\left(Z^{\prime}, \hat{A}_{0}, \hat{B}_{e 0}\right)$ is stabilizable and from the (2,2)-block of (4.24) and $Z^{\prime} Y=Y-Y X Y \geqq 0$ since $\rho(X Y) \leqq 1$. The sufficiency of the condition follows from (a) and (b) by using the $(2,2)$ entry of $Q_{a a}(s)$.

4.2. Characterization of all solutions. Theorem 4.2 gives a solution in the case $\left\|R_{a}\right\|_{H}=1$ in descriptor form. All solutions can be generated from $Q_{a a}(s)$ but the more detailed structure is required. To keep the notation simple the simplifying assumption

$$
\left[\begin{array}{ll}
D_{11} & D_{12} \\
D_{21} & D_{22}
\end{array}\right]=\left[\begin{array}{ll}
0 & 0 \\
0 & 0
\end{array}\right]
$$

will be made for this section. Model matching problems arising from $H^{\infty}$ control can in fact always be reduced to this case (see [45]). The main difference in the approach in $\S 3$ is that all solutions to the problem with $R_{a}$ rather than $R$ are determined. We wish to write any solution

$$
\left[\begin{array}{ll}
\bar{Q}_{22} & \bar{Q}_{23} \\
\bar{Q}_{32} & \bar{Q}_{33}
\end{array}\right]
$$

as

$$
\left[\begin{array}{ll}
\bar{Q}_{22} & \bar{Q}_{23} \\
\bar{Q}_{32} & \bar{Q}_{33}
\end{array}\right]=\left[\begin{array}{ll}
Q_{22} & Q_{23} \\
Q_{32} & Q_{33}
\end{array}\right]+\left[\begin{array}{c}
\tilde{Q}_{24} \\
\tilde{Q}_{34}
\end{array}\right] \Phi\left(I-\tilde{Q}_{44} \Phi\right)^{-1}\left[\begin{array}{ll}
\tilde{Q}_{42} & \tilde{Q}_{43}
\end{array}\right]
$$

for $\|\Phi\|_{\infty} \leqq 1, \Phi \in H_{\infty}^{+}$. We need to show that such a $\Phi$ exists for all suitable $\bar{Q}_{i j}$, and this is not immediately clear since

$$
\left[\begin{array}{c}
\tilde{Q}_{24} \\
\tilde{Q}_{34}
\end{array}\right] \text { and }\left[\begin{array}{ll}
\tilde{Q}_{42} & \tilde{Q}_{43}
\end{array}\right]
$$


are not invertible. The following technical lemma will be used to check the existence of a $\Phi$ given any

$$
\left[\begin{array}{ll}
\bar{Q}_{22} & \bar{Q}_{23} \\
\bar{Q}_{32} & \bar{Q}_{33}
\end{array}\right]
$$

LEMMA 4.3. Suppose that

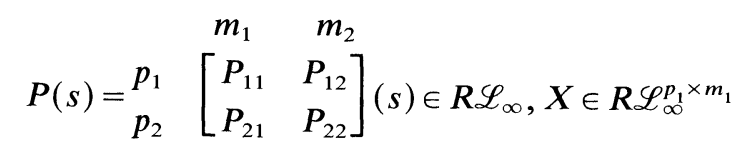

in which $p_{1} \geqq m_{2}, m_{1} \geqq p_{2}$ and $P_{22}(\infty)=0$. Suppose also that $P_{12}$ has a left inverse $P_{12}^{l} \in \mathscr{L}_{\infty}$ and $P_{21}$ has a right inverse $P_{21}^{r} \in \mathscr{L}_{\infty}$. Then if there exists a rational matrix $R \in \mathscr{L}_{\infty}$ with rank $(R) \geqq p_{1}-m_{2}$ for almost all s such that

(i) $R\left[X-P_{11} P_{12}\right]=0$,

and if there exists a rational matrix $S \in \mathscr{L}_{\infty}$ with $\operatorname{rank}(S) \geqq m_{1}-p_{2}$ for almost all s such that

$$
\text { (ii) }\left[\begin{array}{c}
X-P_{11} \\
P_{21}
\end{array}\right] S=0 \text {, }
$$

then there exists a rational matrix $\Phi$ such that $F_{l}(P, \Phi)=X$. More particularly,

$$
\Phi=\left(I+\Psi P_{22}\right)^{-1} \Psi
$$

where

$$
\Psi=P_{12}^{l}\left(X-P_{11}\right) P_{21}^{r} \text {. }
$$

Proof. The idea of the proof is to establish that (i) and (ii) guarantee the existence of a solution $\Psi$ to the equation $X-P_{11}=P_{12} \Psi P_{21}$. We then set $\Psi=\Phi\left(I-P_{22} \Phi\right)^{-1}$ and solve for $\Phi$.

It follows from (i) that $\left(X-P_{11}\right) \in \mathcal{N}(R)$. Since $\operatorname{dim} \mathscr{R}\left(P_{12}\right)=m_{2} \geqq \operatorname{dim} \mathcal{N}(R)$, it follows from (i) that $\left(X-P_{11}\right) \in \mathcal{N}(R) \equiv \mathscr{R}\left(P_{12}\right)$ and therefore that the equation $P_{12} Z=$ $\left(X-P_{11}\right)$ has a solution $Z=P_{12}^{l}\left(X-P_{11}\right)$. In the same way we have $\left(X-P_{11}\right)^{\sim} \in$ $\mathscr{R}\left(P_{21}^{\sim}\right)$. Since $Z^{\sim} \in \mathscr{R}\left(X-P_{11}\right)^{\sim}$, we have $Z^{\sim} \in \mathscr{R}\left(P_{21}^{\sim}\right)$ and consequently $\Psi P_{21}=Z$ has a solution $\Psi=P_{12}^{l}\left(X-P_{11}\right) P_{21}^{r} \in \mathscr{L}_{\infty}$. It follows from $P_{22}(\infty)=0$ and $\Psi=$ $\Phi\left(I-P_{22} \Phi\right)^{-1}$ that $\Phi=\left(I+\Psi P_{22}\right)^{-1} \Psi$ is a proper rational matrix.

The detailed structure of $Q_{a a}(s)$ will now be examined when

$$
\left[\begin{array}{ll}
D_{11} & D_{12} \\
D_{21} & D_{22}
\end{array}\right]=0
$$

In this case we may choose

$$
D_{e}=\left[\begin{array}{llll}
0 & 0 & I & 0 \\
0 & 0 & 0 & I \\
I & 0 & 0 & 0 \\
0 & I & 0 & 0
\end{array}\right]
$$

and substituting (4.33) into (4.26)-(4.28) yields

$$
\hat{C}_{e 0}^{\prime}=\left[\begin{array}{llll}
0 & X C_{2}^{\prime} & X C_{3}^{\prime}-B_{1} & -B_{2}
\end{array}\right] \text {, }
$$




$$
\begin{aligned}
& \hat{B}_{e 0}=\left[\begin{array}{llll}
0 & Y B_{2} & Y B_{3}-C_{1}^{\prime} & -C_{2}^{\prime}
\end{array}\right], \\
& \hat{A_{0}}=-A^{\prime}-Y A X+C_{3}^{\prime} B_{1}^{\prime}+C_{1}^{\prime} B_{3}^{\prime} \text {. }
\end{aligned}
$$

Since (3.51) and (3.52) have an appropriate dual relationship to each other, their solutions may be transformed to the balanced form

$$
X=\left[\begin{array}{cc}
\bar{X} & 0 \\
0 & I_{k}
\end{array}\right], \quad Y=\left[\begin{array}{cc}
\bar{Y} & 0 \\
0 & I_{k}
\end{array}\right], \quad \bar{Z}=I-\bar{X} \bar{Y}
$$

by an appropriate change of basis in the state space of $R(s) ; k$ is the multiplicity of the unit singular value of $\Gamma_{R_{a}^{\sim}}$. This balancing induces the following partitioning on $B_{e}$ and $C_{e}$ in (3.59) and (3.60).

$$
\begin{gathered}
{\left[\begin{array}{l}
B_{e 1} \\
B_{e 2}
\end{array}\right]=\left[\begin{array}{llll}
B_{11} & B_{12} & B_{13} & 0 \\
B_{21} & B_{22} & B_{23} & 0
\end{array}\right],} \\
{\left[\begin{array}{l}
C_{e 1}^{\prime} \\
C_{e 2}^{\prime}
\end{array}\right]=\left[\begin{array}{llll}
C_{11}^{\prime} & C_{12}^{\prime} & C_{13}^{\prime} & 0 \\
C_{21}^{\prime} & C_{22}^{\prime} & C_{23}^{\prime} & 0
\end{array}\right] .}
\end{gathered}
$$

Furthermore if rank $B_{22}=l<k$, then an additional unitary change of basis on the $k$ states can give $B_{22}=\left[\begin{array}{c}B_{221} \\ 0\end{array}\right]$, with $B_{221}$ full rank.

Substitution into (3.49) and (3.50) gives

$$
\begin{aligned}
& {\left[\begin{array}{l}
B_{13} \\
B_{23}
\end{array}\right]=\left[\begin{array}{c}
\bar{X} C_{11}^{\prime} \\
C_{21}^{\prime}
\end{array}\right],} \\
& {\left[\begin{array}{l}
C_{13}^{\prime} \\
C_{23}^{\prime}
\end{array}\right]=\left[\begin{array}{c}
\bar{Y} B_{11} \\
B_{21}
\end{array}\right] .}
\end{aligned}
$$

Finally, (3.51) and (3.52) become

$$
\begin{aligned}
&-\left[\begin{array}{ll}
A_{11} & A_{12} \\
A_{21} & A_{22}
\end{array}\right]\left[\begin{array}{cc}
\bar{X} & 0 \\
0 & I_{k}
\end{array}\right]-\left[\begin{array}{cc}
\bar{X} & 0 \\
0 & I_{k}
\end{array}\right]\left[\begin{array}{ll}
A_{11}^{\prime} & A_{21}^{\prime} \\
A_{12}^{\prime} & A_{22}^{\prime}
\end{array}\right]+\left[\begin{array}{ccc}
B_{11} & B_{12} & \bar{X} C_{11}^{\prime} \\
B_{21} & B_{22} & C_{21}^{\prime}
\end{array}\right] \\
& {\left[\begin{array}{ccc}
B_{11} & B_{12} & \bar{X} C_{11}^{\prime} \\
B_{21} & B_{22} & C_{21}^{\prime}
\end{array}\right]^{\prime}=0, }
\end{aligned}
$$

$$
\begin{aligned}
&-\left[\begin{array}{ll}
A_{11}^{\prime} & A_{21}^{\prime} \\
A_{12}^{\prime} & A_{22}^{\prime}
\end{array}\right]\left[\begin{array}{ll}
\bar{Y} & 0 \\
0 & I_{k}
\end{array}\right]-\left[\begin{array}{cc}
\bar{Y} & 0 \\
0 & I_{k}
\end{array}\right]\left[\begin{array}{ll}
A_{11} & A_{12} \\
A_{21} & A_{22}
\end{array}\right]+\left[\begin{array}{lll}
C_{11}^{\prime} & C_{12}^{\prime} & \bar{Y} B_{11} \\
C_{21}^{\prime} & C_{22}^{\prime} & B_{21}
\end{array}\right] \\
& {\left[\begin{array}{ccc}
C_{11}^{\prime} & C_{12}^{\prime} & \bar{Y} B_{11} \\
C_{21}^{\prime} & C_{22}^{\prime} & B_{21}
\end{array}\right]^{\prime}=0 . }
\end{aligned}
$$

The $(2,2)$ blocks of $(4.42)$ and $(4.43)$ establish the following equation:

$$
B_{22} B_{22}^{\prime}=C_{22}^{\prime} C_{22}
$$

and consequently that there exists a matrix $U$ such that

$$
U B_{22}^{\prime}=C_{22}, \quad U:=C_{22}\left(B_{22}^{\prime}\right)^{\dagger}=\left(C_{22}^{\prime}\right)^{\dagger} B_{22} .
$$


It now follows that a descriptor representation for $Q_{a a}(s)$ is given by

$$
\begin{gathered}
{\left[\begin{array}{cc}
\bar{Z}^{\prime} & 0 \\
0 & 0
\end{array}\right]\left[\begin{array}{l}
\dot{x}_{1} \\
\dot{x}_{2}
\end{array}\right]=\left[\begin{array}{cc}
-A_{11}^{\prime}-\bar{Y} A_{11} \bar{X}+C_{13}^{\prime} B_{11}^{\prime}+C_{11}^{\prime} B_{13}^{\prime} & -C_{12}^{\prime} U B_{22}^{\prime} \\
-B_{22} B_{12}^{\prime} & -B_{22} B_{22}^{\prime}
\end{array}\right]\left[\begin{array}{l}
x_{1} \\
x_{2}
\end{array}\right]} \\
+\left[\begin{array}{cccc}
0 & \bar{Y} B_{12} & -\bar{Z}^{\prime} C_{11}^{\prime} & -C_{12}^{\prime} \\
0 & B_{22} & 0 & -B_{22} U^{\prime}
\end{array}\right]\left[\begin{array}{l}
u_{1} \\
u_{2} \\
u_{3} \\
u_{4}
\end{array}\right] \\
{\left[\begin{array}{l}
y_{1} \\
y_{2} \\
y_{3} \\
y_{4}
\end{array}\right]\left[\begin{array}{cc}
0 & 0 \\
C_{12} \bar{X} & U B_{22}^{\prime} \\
-B_{11}^{\prime} \bar{Z}^{\prime} & 0 \\
-B_{12}^{\prime} & -B_{22}^{\prime}
\end{array}\right]\left[\begin{array}{l}
x_{1} \\
x_{2}
\end{array}\right]+\left[\begin{array}{llll}
0 & 0 & I & 0 \\
0 & 0 & 0 & I \\
I & 0 & 0 & 0 \\
0 & I & 0 & 0
\end{array}\right]\left[\begin{array}{l}
u_{1} \\
u_{2} \\
u_{3} \\
u_{4}
\end{array}\right]}
\end{gathered}
$$

on substituting (4.37) to (4.41) into (4.34)-(4.36). The $(2,1)$ block of $(4.46)$ gives

$$
\begin{aligned}
& 0=-B_{22} B_{12}^{\prime} x_{1}-B_{22} B_{22}^{\prime} x_{2}+B_{22} u_{2}-B_{22} U^{\prime} u_{4} \\
& \Rightarrow B_{22}^{\prime} x_{2}=\left(B_{22}\right)^{\dagger}\left(-B_{22} B_{12}^{\prime} x_{1}+B_{22} u_{2}-C_{22}^{\prime} u_{4}\right) .
\end{aligned}
$$

Substituting (4.48) into (4.46) and (4.47) gives the state-space realization,

$$
\begin{aligned}
& {\left[\begin{array}{lll}
Q_{22} & Q_{23} & Q_{24} \\
Q_{32} & Q_{33} & Q_{34} \\
Q_{42} & Q_{43} & Q_{44}
\end{array}\right](s)} \\
& \stackrel{s}{=}\left[\begin{array}{c|ccc} 
& & & \\
\hline C_{12} \bar{X}-U B_{12}^{\prime} & \left(\bar{Z}^{\prime}\right)^{-1}\left(\bar{Y} B_{12}-C_{12}^{\prime} U\right) & -C_{11}^{\prime} & -\left(\bar{Z}^{\prime}\right)^{-1} C_{12}^{\prime}\left(I-C_{22} C_{22}^{\dagger}\right) \\
-B_{11}^{\prime} \bar{Z}^{\prime} & U & 0 & \left(I-C_{22} C_{22}^{\dagger}\right) \\
-\left(I-B_{22}^{\dagger} B_{22}\right) B_{12}^{\prime} & 0 & 0 & 0 \\
- & I-B_{22}^{\dagger} B_{22} & 0 & -B_{22}^{\dagger} C_{22}^{\prime}
\end{array}\right]
\end{aligned}
$$

where

$$
\tilde{A}=\left(\bar{Z}^{\prime}\right)^{-1}\left(-A_{11}^{\prime}-\bar{Y} A_{11} \bar{X}+C_{13}^{\prime} B_{11}^{\prime}+C_{11}^{\prime} B_{13}^{\prime}+C_{12}^{\prime} C_{22}\left(B_{22}^{\prime}\right)^{\dagger} B_{12}^{\prime}\right) \text {. }
$$

Suppose that $\Theta_{1}$ and $\Theta_{3}$ are orthogonal bases for $\mathscr{R}\left(B_{22}^{\prime}\right)$ and $\mathscr{R}\left(C_{22}\right)$, respectively, and that $\Theta_{2}$ and $\Theta_{4}$ are chosen to make $\left[\begin{array}{ll}\Theta_{2} & \Theta_{1}\end{array}\right]$ and $\left[\begin{array}{ll}\Theta_{4} & \Theta_{3}\end{array}\right]$ orthogonal. ${ }^{1}$ If we multiply the last row of $(4.49)$ by $\left[\begin{array}{l}\Theta_{2}^{\prime} \\ \Theta_{1}^{\prime}\end{array}\right]$ and the last column by $\left[\begin{array}{ll}\Theta_{4} & \Theta_{3}\end{array}\right]$, we obtain

$$
\left[\begin{array}{cccc}
Q_{22} & Q_{23} & \tilde{Q}_{24} & 0 \\
Q_{32} & Q_{33} & \tilde{Q}_{34} & 0 \\
\tilde{Q}_{42} & \tilde{Q}_{43} & \tilde{Q}_{44} & 0 \\
0 & 0 & 0 & -I_{l}
\end{array}\right](s)
$$

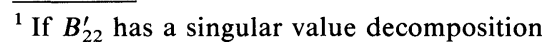

$$
B_{22}^{\prime}=\left[\begin{array}{ll}
Y_{1} & Y_{2}
\end{array}\right]\left[\begin{array}{ll}
0 & 0 \\
0 & \Gamma
\end{array}\right]\left[\begin{array}{l}
U_{1}^{\prime} \\
U_{2}^{\prime}
\end{array}\right],
$$

then $\left[\begin{array}{ll}\Theta_{2} & \Theta_{1}\end{array}\right]=\left[\begin{array}{ll}Y_{1} & Y_{2}\end{array}\right]$ and $\left(B_{22}^{\prime}\right)^{\dagger}=U_{2} \Gamma^{-1} Y_{2}^{\prime}$. 


$$
\stackrel{s}{=}\left[\begin{array}{c|cccc}
\tilde{A} & \left(\bar{Z}^{\prime}\right)^{-1}\left(\bar{Y} B_{12}-C_{12}^{\prime} U\right) & -C_{11}^{\prime} & -\left(\bar{Z}^{\prime}\right)^{-1} C_{12}^{\prime} \Theta_{4} & 0 \\
\hline C_{12} \bar{X}-U B_{12}^{\prime} & U & 0 & \Theta_{4} & 0 \\
-B_{11}^{\prime} \bar{Z}^{\prime} & 0 & 0 & 0 & 0 \\
-\Theta_{2}^{\prime} B_{12}^{\prime} & \Theta_{2}^{\prime} & 0 & 0 & 0 \\
0 & 0 & 0 & 0 & -I_{l}
\end{array}\right]
$$

in which

$$
l=\operatorname{rank}\left(B_{22}\right)=\operatorname{rank}\left(C_{22}\right) \leqq k .
$$

Equation (4.50) has an interesting structure in that it shows how the effective dimension of the free parameter is reduced by $l$. We will examine this point a little further and obtain a constraining equation on the free parameter $\Phi$ which is reminicent of $[16$, eq. (8.69)]. Following that, we will prove that

$$
\left[\begin{array}{cc}
Q_{22} & \tilde{Q}_{24} \\
\tilde{Q}_{42} & \tilde{Q}_{44}
\end{array}\right](s) \stackrel{s}{=}\left[\begin{array}{c|cc}
\tilde{A} & \left(\bar{Z}^{\prime}\right)^{-1}\left(\bar{Y} B_{12}-C_{12}^{\prime} U\right) & -\left(\bar{Z}^{\prime}\right)^{-1} C_{12}^{\prime} \Theta_{4} \\
\hline C_{12} \bar{X}-U B_{12}^{\prime} & U & \Theta_{4} \\
-\Theta_{2}^{\prime} B_{12}^{\prime} & \Theta_{2}^{\prime} & 0
\end{array}\right]
$$

captures all solutions in the optimal case corresponding to $\left\|\Gamma_{R_{a}^{\sim}}\right\|=1$.

Let us write the left-hand side of $(4.50)$ as

$$
\left[\begin{array}{l}
y_{1} \\
y_{2} \\
y_{3}
\end{array}\right]=\left[\begin{array}{ccc}
Q_{22} & \tilde{Q}_{24} & 0 \\
\tilde{Q}_{42} & \tilde{Q}_{44} & 0 \\
0 & 0 & I_{l}
\end{array}\right]\left[\begin{array}{l}
u_{1} \\
u_{2} \\
u_{3}
\end{array}\right]
$$

and allow this to induce the following partitioning on the free contraction $\Phi(s)$

$$
\left[\begin{array}{l}
u_{2} \\
u_{3}
\end{array}\right]=\left[\begin{array}{ll}
\Phi_{11} & \Phi_{12} \\
\Phi_{21} & \Phi_{22}
\end{array}\right]\left[\begin{array}{l}
y_{2} \\
y_{3}
\end{array}\right] .
$$

Since $u_{3}=y_{3}$ we may eliminate this variable in (4.54) to obtain

$$
\begin{aligned}
u_{2}=\left(\Phi_{11}+\Phi_{12}(I\right. & \left.\left.-\Phi_{22}\right)^{-1} \Phi_{21}\right) y_{2} \text { if } \operatorname{det}\left(I-\Phi_{22}(\infty)\right) \neq 0 \text { is assumed } \\
= & \left(F_{l}\left\{\left[\begin{array}{ll}
\Phi_{11} & \Phi_{12} \\
\Phi_{21} & \Phi_{22}
\end{array}\right], I_{l}\right\}\right) y_{2}=\tilde{\Phi} y_{2}
\end{aligned}
$$

in which $\|\tilde{\Phi}\| \leqq 1$ by Redheffer's theorem [42]. A class of solutions is therefore given by

$$
Q=F_{l}\left\{\left[\begin{array}{ll}
Q_{22} & \tilde{Q}_{24}^{\prime} \\
\tilde{Q}_{42} & \tilde{Q}_{44}
\end{array}\right], \tilde{\Phi}\right\} .
$$

Transforming back to the coordinates in (4.53) gives

$$
\Phi=U\left[\begin{array}{ll}
\Theta_{2} & \Theta_{1}
\end{array}\right]\left[\begin{array}{ll}
\tilde{\Phi} & 0 \\
0 & I_{l}
\end{array}\right]\left[\begin{array}{l}
\Theta_{2}^{\prime} \\
\Theta_{1}
\end{array}\right]
$$

and (4.57) $B_{22}^{\prime}$

$$
\Rightarrow \Phi B_{22}^{\prime}=C_{22},
$$

which is a linear constraint on $\Phi$ and similar to that obtained in [16]. 
We are now in a position to state and prove the main result of this section.

THEOREM 4.4. Let $R_{i j} \in \mathscr{R} \mathscr{H}_{\infty}^{-, p_{i}, m_{j}}$ for $i, j=1,2$ have the realization

$$
\left[\begin{array}{ll}
R_{11} & R_{12} \\
R_{21} & R_{22}
\end{array}\right] \stackrel{s}{=}\left[\begin{array}{c|cc}
A & B_{1} & B_{2} \\
\hline C_{1} & 0 & 0 \\
C_{2} & 0 & 0
\end{array}\right]
$$

with $\operatorname{Re} \lambda_{i}(A)>0$.

If the conditions of Corollary 3.5(i) are met, then every $Q \in \mathscr{R}_{\infty}^{+}$satisfying

$$
\left\|\left[\begin{array}{cc}
R_{11} & R_{12} \\
R_{21} & R_{22}+Q
\end{array}\right]\right\|_{\infty} \leqq 1
$$

is given by

$$
Q=F_{l}\left(\left[\begin{array}{ll}
Q_{22} & \tilde{Q}_{24} \\
\tilde{Q}_{42} & \tilde{Q}_{44}
\end{array}\right], \Phi\right) \text { for some } \Phi \in \mathscr{R} \mathscr{H}_{\infty}^{+} \text {with }\|\Phi\|_{\infty} \leqq 1
$$

in which

$$
\left[\begin{array}{ll}
Q_{22} & \tilde{Q}_{24} \\
\tilde{Q}_{42} & \tilde{Q}_{44}
\end{array}\right]
$$

has a state-space realization given by (4.52).

Proof. The proof can be deduced using arguments similar to those given in the proof of Theorem 3.6. We begin by making two preliminary observations. First, it is an immediate consequence of Redheffers theorem that (4.59) captures a class of solutions. Second,

$$
\left[\begin{array}{ccc}
R_{11} & R_{12} & R_{13} \\
R_{21} & R_{22}+\bar{Q}_{22} & R_{23}+\bar{Q}_{23} \\
R_{31} & R_{32}+\bar{Q}_{32} & R_{33}+\bar{Q}_{33}
\end{array}\right]\left[\begin{array}{cc}
R_{11}^{\sim} & V_{11} \\
R_{12}^{\sim} & V_{21} \\
R_{13}^{\sim} & V_{31}
\end{array}\right]=\left[\begin{array}{cc}
I & U_{11}(-s) \\
0 & U_{21}(-s) \\
0 & U_{31}(-s)
\end{array}\right]
$$

for any

$$
Q=\left[\begin{array}{ccc}
0 & 0 & 0 \\
0 & \bar{Q}_{22} & \bar{Q}_{23} \\
0 & \bar{Q}_{32} & \bar{Q}_{33}
\end{array}\right]
$$

such that $\left\|R_{a}+Q\right\|_{\infty}=\left\|R_{a}^{\sim}\right\|_{H}=1$ where $V(s)$ and $U(-s)$ are the maximal Schmidt vectors of the Hankel operator $\Gamma_{R_{a}^{\sim}}$ [14], [18]. Since

$$
\left[\begin{array}{lll}
R_{11} & R_{12} & R_{13} \\
V_{11}^{\sim} & V_{21}^{\sim} & V_{31}^{\sim}
\end{array}\right](\infty)=\left[\begin{array}{ccc}
0 & 0 & I \\
C_{21} & C_{22} & C_{23}
\end{array}\right]
$$

it is immediate that

$$
p_{1}+k \geqq \operatorname{rank}\left[\begin{array}{cc}
R_{12}^{\sim} & V_{21} \\
R_{13}^{\sim} & V_{31}
\end{array}\right] \geqq p_{1}+\operatorname{rank}\left(C_{22}\right)
$$

for almost all $s ; k$ is the multiplicity of the unit Hankel singular value of $\Gamma_{R_{a}^{\sim}}$. Similarly, since $\left\|R_{a a}+Q_{a a}\right\|_{\infty}=\left\|R_{a a}^{\sim}\right\|_{H}=1$

$$
\left[\begin{array}{ccc}
0 & 0 & 0 \\
0 & \bar{Q}_{22}-Q_{22} & \bar{Q}_{23}-Q_{23} \\
0 & \bar{Q}_{32}-Q_{32} & \bar{Q}_{33}-Q_{33} \\
0 & \tilde{Q}_{42} & \tilde{Q}_{43}
\end{array}\right]\left[\begin{array}{cc}
R_{11}^{\sim} & V_{11} \\
R_{12}^{\sim} & V_{21} \\
R_{13}^{\sim} & V_{31}
\end{array}\right]=0 .
$$


In the same way we have that

$$
\left[\begin{array}{ccc}
R_{11}^{\sim} & R_{21}^{\sim} & R_{31}^{\sim} \\
U_{11}^{\sim} & U_{21}^{\sim} & U_{31}^{\sim}
\end{array}\right]\left[\begin{array}{cccc}
0 & 0 & 0 & 0 \\
0 & \bar{Q}_{22}-Q_{22} & \bar{Q}_{23}-Q_{23} & \tilde{Q}_{24} \\
0 & \bar{Q}_{32}-Q_{32} & \bar{Q}_{33}-Q_{33} & \tilde{Q}_{34}
\end{array}\right]=0
$$

and

$$
\operatorname{rank}\left[\begin{array}{ccc}
R_{11}^{\sim} & R_{21}^{\sim} & R_{31}^{\sim} \\
U_{11}^{\sim} & U_{21}^{\sim} & U_{31}^{\sim}
\end{array}\right] \geqq m_{1}+\operatorname{rank}\left(B_{22}\right) .
$$

We now show that $\left[\begin{array}{c}\tilde{Q}_{24} \\ \tilde{Q}_{34}\end{array}\right]$ has an asymptotically stable left inverse while $\left[\begin{array}{ll}\tilde{Q}_{42} & \tilde{Q}_{43}\end{array}\right]$ has an asymptotically stable right inverse. Substituting from (4.50) gives

$$
\left[\begin{array}{c}
\tilde{Q}_{24} \\
\tilde{Q}_{34}
\end{array}\right] \stackrel{s}{=}\left[\begin{array}{c|c}
\tilde{A} & -\left(\bar{Z}^{\prime}\right)^{-1} C_{12}^{\prime} \Theta_{4} \\
\hline C_{12} \bar{X}-U B_{12}^{\prime} & \Theta_{4} \\
-B_{11}^{\prime} \bar{Z}^{\prime} & 0
\end{array}\right]
$$

and a simple calculation yields ${ }^{2}$

$$
\begin{gathered}
{\left[\begin{array}{c}
\tilde{Q}_{24} \\
\tilde{Q}_{34}
\end{array}\right]^{l} \stackrel{s}{=}\left[\begin{array}{c|cc}
-\left(\bar{Z}^{\prime}\right)^{-1} A_{11} \bar{Z} & \left(\bar{Z}^{\prime}\right)^{-1}\left[\begin{array}{ll}
C_{12}^{\prime} & C_{13}^{\prime}
\end{array}\right] \\
\hline \Theta_{4}^{\prime}\left[C_{12} \bar{X}-U B_{12}^{\prime}\right] & \Theta_{4}^{\prime} & 0
\end{array}\right] .} \\
{\left[\begin{array}{cc}
\tilde{Q}_{42} & \tilde{Q}_{43}
\end{array}\right] \stackrel{(\tilde{A}}{=}\left[\begin{array}{ccc}
\left(\bar{Z}^{\prime}\right)^{-1}\left[\bar{Y} B_{12}-C_{12}^{\prime} U\right]-C_{11}^{\prime} \\
\hline-\left[\begin{array}{ll}
\Theta_{2}^{\prime} & 0
\end{array}\right]\left[\begin{array}{l}
B_{12}^{\prime} \\
B_{13}^{\prime}
\end{array}\right] & \Theta_{2}^{\prime} & 0
\end{array}\right]}
\end{gathered}
$$

has a right inverse

$$
\left[\begin{array}{ll}
\tilde{Q}_{42} & \tilde{Q}_{43}
\end{array}\right]^{r} \stackrel{s}{=}\left[\begin{array}{c|c}
-A_{11}^{\prime} & -\left(\bar{Z}^{\prime}\right)^{-1}\left[\bar{Y} B_{12}-C_{12}^{\prime} U\right] \Theta_{2} \\
\hline B_{12}^{\prime} & \Theta_{2} \\
B_{13}^{\prime} & 0
\end{array}\right] .
$$

Since $\lambda_{i}\left(A_{11}\right)>0$, the left and right inverses in (4.66) and (4.68) are asymptotically stable. Furthermore,

$$
\begin{gathered}
\operatorname{rank}\left[\begin{array}{c}
\tilde{Q}_{24} \\
\tilde{Q}_{34}
\end{array}\right]=p_{2}-\operatorname{rank}\left(B_{22}\right) \quad \text { for all } \operatorname{Re}(s) \geq 0, \\
\operatorname{rank}\left[\begin{array}{cc}
\tilde{Q}_{42} & \tilde{Q}_{43}
\end{array}\right]=m_{2}-\operatorname{rank}\left(B_{22}\right) \quad \text { for all } \operatorname{Re}(s) \geqq 0 .
\end{gathered}
$$

Hence, we may invoke Lemma 4.3 and the all-pass character of $E_{a a}$ to establish the existence of a $\|\Phi\|_{\infty} \leqq 1$ such that

$$
\left[\begin{array}{ll}
\bar{Q}_{22} & \bar{Q}_{23} \\
\bar{Q}_{32} & \bar{Q}_{33}
\end{array}\right]=F_{l}\left(\left[\begin{array}{lll}
Q_{22} & Q_{23} & \tilde{Q}_{24} \\
Q_{32} & Q_{33} & \tilde{Q}_{34} \\
\tilde{Q}_{42} & \tilde{Q}_{43} & \tilde{Q}_{44}
\end{array}\right] ; \Phi\right)
$$

It remains for us to show that any such $\Phi \in \mathscr{B}_{\mathscr{H}_{\infty}^{+}}^{+}$. It follows immediately that

$$
\begin{gathered}
\Psi=\left[\begin{array}{c}
\tilde{Q}_{24} \\
\tilde{Q}_{34}
\end{array}\right]^{l}\left[\begin{array}{cc}
\bar{Q}_{22}-Q_{22} & \bar{Q}_{23}-Q_{23} \\
\bar{Q}_{32}-Q_{32} & \bar{Q}_{33}-Q_{33}
\end{array}\right]\left[\begin{array}{ll}
\tilde{Q}_{42} & \tilde{Q}_{43}
\end{array}\right]^{r} \\
G(s) \stackrel{s}{=}\left[\begin{array}{cc}
A & B D \\
C & D
\end{array}\right] \Rightarrow G^{l}(s) \stackrel{s}{=}\left[\begin{array}{cc}
A-B C & -B \\
D^{l} C & D^{l}
\end{array}\right]
\end{gathered}
$$

and a dual result is clearly true in the case of a right inverse. 
is stable. Now suppose that $\Phi$ has a coprime factorization $\Phi=N D^{-1}$ with $N, D \in \mathscr{R} \mathscr{H}_{\infty}^{+}$. Since $\Psi=\Phi\left(I-Q_{44} \Phi\right)^{-1}$ is stable, and $\Psi=N\left(D-Q_{44} N\right)^{-1}$ is a coprime factorization, it follows that $\left(D-Q_{44} N\right)$ is outer. We may now deduce from

$$
\operatorname{det}\left(D-\tilde{Q}_{44} N\right)(j \omega)=\operatorname{det} D(j \omega) \cdot \operatorname{det}\left(I-\tilde{Q}_{44} \Phi\right)(j \omega)
$$

and $\left\|\tilde{Q}_{44} \Phi\right\|_{\infty}<1$ that the winding number of det $D(j \omega)$ around the origin is zero. This means that $D$ is outer and therefore that $\Phi=N D^{-1} \in \mathscr{R} \mathscr{H}_{\infty}^{+}$as required.

5. A representation formula for all internally stabilizing controllers that satisfy a closed-loop $\boldsymbol{H}^{\infty}$ norm constraint. In this section we will show how the solution to the four block problem given in $\S \S 3$ and 4 may be used to solve a rational $\mathscr{H}^{\infty}$ control problem. Suppose we are given

$$
P=\left[\begin{array}{cc}
l & m \\
P_{11} & P_{12} \\
P_{21} & P_{22}
\end{array}\right] p \underset{s}{p}\left[\begin{array}{c|cc}
A & l & m \\
\hline C_{1} & D_{11} & B_{2} \\
C_{2} & D_{21} & D_{22}
\end{array}\right] \begin{aligned}
& n \\
& p \\
& q
\end{aligned}
$$

in which $p \geqq m$ and $l \geqq q$, and that we seek all controllers that stabilize $F_{l}(P, K)$ and that satisfy the norm constraint

$$
\left\|F_{l}(P, K)\right\|_{\infty} \leqq \gamma .
$$

We will make a number of assumptions regarding (5.1), the last of which is temporary and is removed in our later development.

(i) $\left(A, B_{2}, C_{2}\right)$ is stabilizable and detectable.

(ii) $\operatorname{rank}\left(D_{12}\right)=m$ and $\operatorname{rank}\left(D_{21}\right)=q$.

(iii) $\quad \operatorname{rank}\left(\left[\begin{array}{cc}j \omega I-A & -B_{2} \\ C_{1} & D_{12}\end{array}\right]\right)=n+m$ for all real $\omega$.

(iv) $\quad \operatorname{rank}\left(\left[\begin{array}{cc}j \omega I-A & -B_{1} \\ C_{2} & D_{21}\end{array}\right]\right)=n+q$ for all real $\omega$.

(v) Temporary assumption. $D_{11}=0$ and $D_{22}=0$.

Additionally, the results that follow presume that the problem has been scaled so that the columns of $D_{12}$ and $D_{21}^{\prime}$ are orthogonal. This is always possible by assumption (ii) (see [43]). We will introduce $D_{\perp}$ and $\tilde{D}_{\perp}$ which make $\left[D_{\perp} D_{12}\right]$ and $\left[\tilde{D}_{\perp}^{\prime} D_{21}^{\prime}\right]$ unitary.

The main results of this section will now be stated, and their proofs will be given in the following three sections. Theorem 5.1 gives necessary and sufficient conditions for the existence of a solution, while Theorem 5.2 characterizes all the solutions. Assumption (v) will be removed in $\S 5.3$.

THEOREM 5.1. Suppose that $P(s)$ is given by (5.1) and that assumptions (i)-(v) are satisfied. Then for any $\gamma>0$ there exists an internally stabilizing controller $K(s)$ such that $\left\|F_{l}(P, K)\right\|_{\infty} \leqq \gamma$, if and only if:

(i) There exists

$$
\left[\begin{array}{l}
X_{\infty 1} \\
X_{\infty 2}
\end{array}\right] \in \mathscr{R}^{2 n \times n} \text { of } \operatorname{rank}(n)
$$

such that

$$
\begin{gathered}
H_{\infty}\left[\begin{array}{l}
X_{\infty 1} \\
X_{\infty 2}
\end{array}\right]= \\
{\left[\begin{array}{l}
X_{\infty 1} \\
X_{\infty 2}
\end{array}\right] T_{x}, \quad \operatorname{Re} \lambda_{i}\left(T_{x}\right) \leqq 0 \quad \forall i} \\
X_{\infty 1}^{\prime} X_{\infty 2}=X_{\infty 2}^{\prime} X_{\infty 1}
\end{gathered}
$$


where

$$
H_{\infty}=\left[\begin{array}{cc}
A-B_{2} D_{12}^{\prime} C_{1} & \gamma^{-2} B_{1} B_{1}^{\prime}-B_{2} B_{2}^{\prime} \\
-C_{1}^{\prime} D_{\perp} D_{\perp}^{\prime} C_{1} & -\left(A-B_{2} D_{12}^{\prime} C_{1}\right)^{\prime}
\end{array}\right] .
$$

(ii) There exists

$\left[\begin{array}{c}Y_{\infty 1} \\ Y_{\infty 2}\end{array}\right] \in \mathscr{R}^{2 n \times n}$ of rank $(n)$ such that

$$
\begin{gathered}
J_{\infty}\left[\begin{array}{l}
Y_{\infty 1} \\
Y_{\infty 2}
\end{array}\right]=\left[\begin{array}{l}
Y_{\infty 1} \\
Y_{\infty 2}
\end{array}\right] T_{Y}, \quad \operatorname{Re} \lambda_{i}\left(T_{Y}\right) \leqq 0 \quad \forall i \\
Y_{\infty 1}^{\prime} Y_{\infty 2}=Y_{\infty 2}^{\prime} Y_{\infty 1}
\end{gathered}
$$

where

$$
J_{\infty}=\left[\begin{array}{cc}
\left(A-B_{1} D_{21}^{\prime} C_{2}\right)^{\prime} & \gamma^{-2} C_{1}^{\prime} C_{1}-C_{2}^{\prime} C_{2} \\
-B_{1} \tilde{D}_{\perp}^{\prime} \tilde{D}_{\perp} B_{1}^{\prime} & -\left(A-B_{1} D_{21}^{\prime} C_{2}\right)
\end{array}\right],
$$

and

$$
\left[\begin{array}{cc}
X_{\infty 22}^{\prime} X_{\infty 1} & \gamma^{-1} X_{\infty 22}^{\prime} Y_{\infty 22}^{\prime} \\
\gamma^{-1} Y_{\infty 2}^{\prime} X_{\infty 2} & Y_{\infty 2}^{\prime} Y_{\infty 1}
\end{array}\right] \geqq 0 .
$$

Remark 5.1 (connections with previous results). In the case where $X_{\infty 1}$ and $Y_{\infty 1}$ are invertible we have $(5.9) \geqq 0$ if and only if

$$
\begin{aligned}
& {\left[\begin{array}{cc}
\left(X_{\infty 11}^{\prime}\right)^{-1} & 0 \\
0 & \left(Y_{\infty 1}^{\prime}\right)^{-1}
\end{array}\right](5.9)\left[\begin{array}{cc}
X_{\infty 1}^{-1} & 0 \\
0 & Y_{\infty 1}^{-1}
\end{array}\right]=\left[\begin{array}{cc}
X_{\infty} & \gamma^{-1} X_{\infty} Y_{\infty} \\
\gamma^{-1} Y_{\infty} X_{\infty} & Y_{\infty}
\end{array}\right] \geqq 0} \\
& \Rightarrow\left[\begin{array}{cc}
I & 0 \\
\gamma^{-1} Y_{\infty} & I
\end{array}\right]\left[\begin{array}{cc}
X_{\infty} & 0 \\
0 & Y_{\infty}\left(I-\gamma^{-2} X_{\infty} Y_{\infty}\right)
\end{array}\right]\left[\begin{array}{cc}
I & \gamma^{-1} Y_{\infty} \\
0 & I
\end{array}\right] \geqq 0 .
\end{aligned}
$$

Thus (5.9) is equivalent to the three conditions (1) $X_{\infty} \geqq 0$, (2) $Y_{\infty} \geqq 0$, and (3) $\rho\left(X_{\infty} Y_{\infty}\right) \leqq \gamma^{2}$. These last three conditions are given in [11] and [20] in the suboptimal case. The optimal cases in which $X_{\infty}$ and $Y_{\infty}$ exist were considered in [33] where a connection with vector interpolation is given.

The conditions of Theorem 5.1 treat the cases in which $X_{\infty 1}$ and/or $Y_{\infty 1}$ are singular. Examples of this type of optimality are given in [27].

THEOREM 5.2. If the conditions of Theorem 5.1 are satisfied, then all internally stabilizing controllers $K$ that satisfy $\left\|F_{l}(P, K)\right\|_{\infty} \leqq \gamma$ are given by

(5.10) $K=F_{l}(\mathscr{K}, U) \quad$ with $U \in \mathscr{R} \mathscr{H}_{+}^{\infty},\|U\|_{\infty} \leqq \gamma, \quad \operatorname{det}\left(I-\mathscr{K}_{22}(\infty) U(\infty)\right) \neq 0$

where

$$
\begin{gathered}
\mathscr{K}(s)=\left[\begin{array}{ll}
0 & I \\
I & 0
\end{array}\right]+\left[\begin{array}{l}
C_{k 1} \\
C_{k 2}
\end{array}\right]\left(s E_{k}-A_{k}\right)^{\not \#}\left[\begin{array}{ll}
B_{k 1} & B_{k 2}
\end{array}\right] \\
E_{k}=Y_{\infty 1}^{\prime} X_{\infty 1}-\gamma^{-2} Y_{\infty 2}^{\prime} X_{\infty 2} \\
B_{k 1}=Y_{\infty 1}^{\prime} B_{1} D_{21}^{\prime}+Y_{\infty 2}^{\prime} C_{2}^{\prime} \\
B_{k 2}=Y_{\infty 1}^{\prime} B_{2}+\gamma^{-2} Y_{\infty 2}^{\prime} C_{1}^{\prime} D_{12} \\
C_{k 1}=-D_{12}^{\prime} C_{1} X_{\infty 1}-B_{2}^{\prime} X_{\infty 2} \\
C_{k 2}=-C_{2} X_{\infty 1}-\gamma^{-2} D_{21} B_{1}^{\prime} X_{\infty 2} \\
A_{k}=E_{k} T_{x}+B_{k 1} C_{k 2} \\
=T_{Y}^{\prime} E_{k}+B_{k 2} C_{k 1}
\end{gathered}
$$


Remark 5.2 (computations, degree reduction, and the effective dimension of the free parameter). There are two possible consequences of $E_{k}$ being singular. First, $s E_{k}-A_{k}$ may have eigenvalues at infinity which do not appear as poles of $\mathscr{K}(s)$, and second, $s E_{k}-A_{k}$ may be singular for all values of $s$. The same remarks applied to the realization of $Q_{a a}(s)$ given in $\S 4$, and this was shown to be reducible to a standard state-space realization. The reason was twofold: First, $E_{a a}=R_{a a}+Q_{a a}$ satisfies the descriptor all-pass equations of Theorem 4.1, and second the realization of $Q_{a a}$ has a particular structure as shown in $\S 4.3$. It is easy to use the linear fractional relationship between $\mathscr{K}(s)$ and $Q_{a a}(s)$ to verify that the calculations, which were previously applied to $Q_{a a}(s)$ in $\S 4$, are applicable to the realization of $\mathscr{K}(s)$, and these are now outlined. Suppose orthogonal changes of basis $U$ and $V$ are chosen so that $U E_{k} V=\left[\begin{array}{cc}\hat{E}_{k} & 0 \\ 0^{k} & 0\end{array}\right]$ in which $\hat{E}_{k}$ is nonsingular. Then (5.13) to (5.17) become

$$
\begin{aligned}
& \hat{A}_{k}=U A_{k} V=\left[\begin{array}{ll}
\hat{A}_{k 11} & \hat{A}_{k 12} \\
\hat{A}_{k 21} & \hat{A}_{k 22}
\end{array}\right], \\
& \hat{B}_{k}=U\left[\begin{array}{ll}
B_{k 1} & B_{k 2}
\end{array}\right]=\left[\begin{array}{ll}
\hat{B}_{k 11} & \hat{B}_{k 12} \\
\hat{B}_{k 21} & \hat{B}_{k 22}
\end{array}\right], \\
& \hat{C}_{k}=\left[\begin{array}{l}
C_{k 1} \\
C_{k 2}
\end{array}\right] V=\left[\begin{array}{ll}
\hat{C}_{k 11} & \hat{C}_{k 12} \\
\hat{C}_{k 21} & \hat{C}_{k 22}
\end{array}\right]
\end{aligned}
$$

in which the partitioning is compatible with that of $\hat{E}_{k}$. As explained in $\S 4$, the state dimension of $\mathscr{K}(s)$ may be reduced by the rank defect of $E_{k}$ by a singular perturbation type procedure. Direct calculation shows that a state-space model that is free of infinite eigenvalues is given by

$$
\begin{aligned}
& A_{k r}=\hat{E}_{k}^{-1}\left[\begin{array}{ll}
I & -\hat{A}_{k 12} \hat{A}_{k 22}^{\dagger}
\end{array}\right] \hat{A}_{k}\left[\begin{array}{l}
I \\
0
\end{array}\right], \\
& {\left[\begin{array}{ll}
B_{k r 1} & B_{k r 2}
\end{array}\right]=\hat{E}_{k}^{-1}\left[\begin{array}{ll}
I & -\hat{A}_{k 12} \hat{A}_{k 22}^{\dagger}
\end{array}\right] \hat{B}_{k} \text {, }} \\
& {\left[\begin{array}{l}
C_{k r 1} \\
C_{k r 2}
\end{array}\right]=\hat{C}_{k}\left[\begin{array}{c}
I \\
-\hat{A}_{k 22}^{\dagger} \hat{A}_{k 21}
\end{array}\right] \text {, }} \\
& {\left[\begin{array}{ll}
D_{k r 11} & D_{k r 12} \\
D_{k r 21} & D_{k r 22}
\end{array}\right]=\left[\begin{array}{ll}
0 & I \\
I & 0
\end{array}\right]-\left[\begin{array}{l}
\hat{C}_{k 12} \\
\hat{C}_{k 22}
\end{array}\right] \hat{A}_{k 22}^{\dagger}\left[\begin{array}{ll}
\hat{B}_{k 21} & \hat{B}_{k 22}
\end{array}\right] \text {. }}
\end{aligned}
$$

To explicitly show the reduction in dimension of the free parameter $U(s)$, we select orthogonal matrices $Y$ and $Z$ (which always exist) such that

$$
\begin{aligned}
& Y\left[\begin{array}{ll}
C_{k r 2} & D_{k r 21}
\end{array}\right]=\left[\begin{array}{cc}
\bar{C}_{k r 2} & \bar{D}_{k r 21} \\
0 & 0
\end{array}\right], \\
& {\left[\begin{array}{c}
B_{k r 2} \\
D_{k r 12}
\end{array}\right] Z=\left[\begin{array}{cc}
\bar{B}_{k r 2} & 0 \\
\bar{D}_{k r 12} & 0
\end{array}\right],} \\
& Y D_{k r 22} Z=\left[\begin{array}{cc}
\bar{D}_{k r 22} & 0 \\
0 & \gamma^{-1} I
\end{array}\right] .
\end{aligned}
$$

Thus

$$
\mathscr{K}(s)=\left[\begin{array}{ll}
D_{k r 11} & \bar{D}_{k r 12} \\
\bar{D}_{k r 21} & \bar{D}_{k r 22}
\end{array}\right]+\left[\begin{array}{l}
C_{k r 1} \\
\bar{C}_{k r 2}
\end{array}\right]\left(s I-A_{k r}\right)^{-1}\left[\begin{array}{ll}
B_{k r 1} & \bar{B}_{k r 2}
\end{array}\right]
$$


5.1. A review of controller parameterization theory. The purpose of this section is to show how the original $H^{\infty}$ control problem may be recast as a four block general distance problem. Our treatment of parameterization theory will be brief, as this material is standard and the details already appear in several places [10], [14], [15], [43]. Since $P_{11}, P_{12}, P_{21}$, and $P_{22}$ share the same state-space it is clear the $K$ stabilizes $F_{l}(P, K)$ if and only if it stabilizes $P_{22}$. Since $\left(A, B_{2}, C_{2}\right)$ is assumed stabilizable and detectable, such controllers always exist. Let

$$
P_{22}=N_{r} D_{r}^{-1}=D_{l}^{-1} N_{l}
$$

be left and right coprime stable rational matrix fraction descriptions of $P_{22}$ and

$$
\left[\begin{array}{cc}
V_{r} & U_{r} \\
-N_{l} & D_{l}
\end{array}\right]\left[\begin{array}{cc}
D_{r} & -U_{l} \\
N_{r} & V_{l}
\end{array}\right]=\left[\begin{array}{ll}
I & 0 \\
0 & I
\end{array}\right]
$$

the corresponding Bezout identities. All the matrices in (5.31) belong to $\mathscr{H}_{+}^{\infty}$, and the set of all compensators which stabilize $P_{22}$ and thus also $P$ are given by [43]

$$
K=F_{l}\left(K_{0}, Q\right), \quad Q \in \mathscr{H}_{+}^{\infty}
$$

where

$$
K_{0}(s)=\left[\begin{array}{cc}
-V_{r}^{-1} U_{r} & -V_{r}^{-1} \\
V_{l}^{-1} & V_{l}^{-1} N_{r}
\end{array}\right] .
$$

Since

$$
K\left(I-P_{22} K\right)^{-1}=\left(-D_{r} Q-U_{l}\right) D_{l}
$$

we obtain

$$
\begin{aligned}
\mathscr{R}(s) & =P_{11}+P_{12} K\left(I-P_{22} K\right)^{-1} P_{21} \\
& =\left(P_{11}-P_{12} U_{l} D_{l} P_{21}\right)-\left(P_{12} D_{r}\right) Q\left(D_{l} P_{21}\right) \\
& =T_{11}+T_{12} Q T_{21},
\end{aligned}
$$

which is an affine parameterization of all internally stable closed loops.

Since $\left(A, B_{2}\right)$ is stabilizable there exists a state feedback matrix $F$ such that $A-B_{2} F$ is stable. Similarly, since $\left(C_{2}, A\right)$ is detectable there exists an output injection matrix $H$ such that $A-H C_{2}$ is stable. Given any such pair of stabilizing matrices $F$ and $H$, the right and left coprime factorizations of $P_{22}$ together with the solutions of the Bezout identities are given by [10], [14], [15], and [43]

$$
\left[\begin{array}{cc}
D_{r} & -U_{l} \\
N_{r} & V_{l}
\end{array}\right] \stackrel{s}{=}\left[\begin{array}{c|cc}
A-B_{2} F & B_{2} & H \\
\hline-F & I & 0 \\
C_{2} & 0 & I
\end{array}\right]
$$

and

$$
\left[\begin{array}{cc}
V_{r} & U_{r} \\
-N_{l} & D_{l}
\end{array}\right]^{s}=\left[\begin{array}{c|cc}
A-H C_{2} & B_{2} & H \\
\hline F & I & 0 \\
-C_{2} & 0 & I
\end{array}\right] .
$$

Substituting (5.35) and (5.36) into (5.33) yields

$$
K_{0}(s) \stackrel{s}{=}\left[\begin{array}{c|cc}
A-B_{2} F-H C_{2} & H & B_{2} \\
\hline-F & 0 & I \\
-C_{2} & I & 0
\end{array}\right]
$$


after simplification. We will now make the following specific choices of the stabilizing matrices $F$ and $H$ which will lead to all-pass properties in $T_{12}$ and $T_{21}$ in (5.34). Specifically, we define $F$ and $H$ by

$$
\begin{aligned}
& F=D_{12}^{\prime} C_{1}+B_{2}^{\prime} X, \\
& H=B_{1} D_{21}^{\prime}+Y C_{2}^{\prime}
\end{aligned}
$$

where $X$ and $Y$ are the unique stabilizing solutions to

$$
X\left(A-B_{2} D_{12}^{\prime} C_{1}\right)+\left(A-B_{2} D_{12}^{\prime} C_{1}\right)^{\prime} X-X B_{2} B_{2}^{\prime} X+C_{1}^{\prime} D_{\perp} D_{\perp}^{\prime} C_{1}=0
$$

and

$$
Y\left(A-B_{1} D_{21}^{\prime} C_{2}\right)^{\prime}+\left(A-B_{1} D_{21}^{\prime} C_{2}\right) Y-Y C_{2}^{\prime} C_{2} Y+B_{1} \tilde{D}_{\perp}^{\prime} \tilde{D}_{\perp} B_{1}^{\prime}=0 .
$$

Direct substitution into (5.34) leads to

$$
\left[\begin{array}{ccc}
T_{11} & T_{\perp} & T_{12} \\
\tilde{T}_{\perp} & 0 & 0 \\
T_{21} & 0 & 0
\end{array}\right](s) \stackrel{s}{=}\left[\begin{array}{cc|ccc}
A-B_{2} F & B_{2} F & B_{1} & -X^{\dagger} C_{1}^{\prime} D_{\perp} & B_{2} \\
0 & A-H C_{2} & B_{1}-H D_{21} & 0 & 0 \\
\hline C_{1}-D_{12} F & D_{12} F & 0 & D_{\perp} & D_{12} \\
0 & -\tilde{D}_{\perp} B_{1}^{\prime} Y^{\dagger} & \tilde{D}_{\perp} & 0 & 0 \\
0 & C_{2} & D_{21} & 0 & 0
\end{array}\right] .
$$

With this particular choice of $F$ and $H,\left[T_{\perp} \mid T_{12}\right]$ and $\left[\tilde{T}_{\perp}^{\sim} \mid T_{21}^{\sim}\right]$ are all-pass [10], [14], [15], [43]. We call $T_{\perp}(s)$ and $\tilde{T}_{\perp}(s)$ all-pass extensions of $T_{12}$ and $T_{21}$, respectively, and this all-pass property allows us to write

$$
\left\|T_{11}-\left[\begin{array}{ll}
T_{\perp} & T_{12}
\end{array}\right]\left[\begin{array}{cc}
0 & 0 \\
0 & Q
\end{array}\right]\left[\begin{array}{c}
\tilde{T}_{\perp} \\
T_{21}
\end{array}\right]\right\|_{\infty}=\| \begin{array}{cc}
R_{11} & R_{12} \\
R_{21} & R_{22}-Q \|_{\infty}
\end{array}
$$

where

$$
R(s)=\left[\begin{array}{ll}
R_{11} & R_{12} \\
R_{21} & R_{22}
\end{array}\right]=\left[\begin{array}{c}
T_{\perp}^{\sim} \\
T_{12}^{\sim}
\end{array}\right] T_{11}\left[\begin{array}{ll}
\tilde{T}_{\perp}^{\sim} & T_{21}^{\sim}
\end{array}\right] .
$$

Substituting (5.42) into (5.44) gives

$$
R(s) \stackrel{s}{=}\left[\begin{array}{cc|cc}
-\left(A-B_{2} F\right)^{\prime} & X B_{1}\left(B_{1}-H D_{21}\right)^{\prime} & X B_{1} \tilde{D}_{\perp}^{\prime} & X B_{1} D_{21}^{\prime} \\
0 & -\left(A-H C_{2}\right)^{\prime} & Y^{\dagger} B_{1} \tilde{D}_{\perp}^{\prime} & -C_{2}^{\prime} \\
\hline D_{\perp}^{\prime} C_{1} X^{\dagger} & 0 & 0 & 0 \\
-B_{2}^{\prime} & F Y & 0 & 0
\end{array}\right],
$$

which describes the four block problem to be solved.

In the case where $X$ and/or $Y$ are singular, this realization will not be minimal and it will be convenient to delete the nonminimal states as follows [31], [32]. Let

$$
\begin{aligned}
& X=U\left[\begin{array}{cc}
X_{1} & 0 \\
0 & 0
\end{array}\right] U^{\prime}, \quad X_{1}>0 \quad \text { where } U=\left[\begin{array}{ll}
U_{1} & U_{2}
\end{array}\right] \text { is unitary; } \\
& Y=V\left[\begin{array}{cc}
Y_{1} & 0 \\
0 & 0
\end{array}\right] V^{\prime}, \quad Y_{1}>0 \quad \text { where } V=\left[\begin{array}{ll}
V_{1} & V_{2}
\end{array}\right] \text { is unitary. }
\end{aligned}
$$

The Riccati equations for $X$ and $Y$ then give that

$$
\begin{aligned}
& U_{1}^{\prime}\left(A-B_{2} D_{12}^{\prime} C_{1}\right) U_{2}=U_{1}^{\prime}\left(A-B_{2} F\right) U_{2}=0, \quad D_{\perp}^{\prime} C_{1} U_{2}=0 \\
& V_{2}^{\prime}\left(A-B_{1} D_{21}^{\prime} C_{2}\right) V_{1}=V_{2}^{\prime}\left(A-H C_{2}\right) V_{1}=0, \quad V_{2}^{\prime} B_{1} \tilde{D}_{\perp}^{\prime}=0 \\
& B_{1}-H D_{21}=B_{1} \tilde{D}_{\perp}^{\prime} \tilde{D}_{\perp}-Y C_{2}^{\prime} D_{21} \Rightarrow V_{2}^{\prime}\left(B_{1}-H D_{21}\right)=0 \\
& C_{1}-D_{12} F=D_{\perp} D_{\perp}^{\prime} C_{1}-D_{12} B_{2}^{\prime} X \Rightarrow\left(C_{1}-D_{12} F\right) U_{2}=0
\end{aligned}
$$


The state transformation $\left[\begin{array}{cc}U^{\prime} & 0 \\ 0 & V^{\prime}\end{array}\right]$ applied to $R(s)$ then exhibits the nonminimal modes which may be removed, and this followed by the state transformation $\left[\begin{array}{cc}X_{1}^{-1} & 0 \\ 0 & Y_{1}\end{array}\right]$ gives

$$
R(s)=\left[\begin{array}{c|cc}
A_{R} & B_{R 1} & B_{R 2} \\
\hline C_{R 1} & 0 & 0 \\
C_{R 2} & 0 & 0
\end{array}\right] \stackrel{s}{=}\left[\begin{array}{cc|cc}
-A_{F}^{\prime} & U_{1}^{\prime} B_{1} B_{1 H}^{\prime} & U_{1}^{\prime} B_{1} \tilde{D}_{\perp}^{\prime} & U_{1}^{\prime} B_{1} D_{21}^{\prime} \\
0 & -A_{H}^{\prime} & V_{1}^{\prime} B_{1} \tilde{D}_{\perp}^{\prime} & -Y_{1} V_{1}^{\prime} C_{2}^{\prime} \\
\hline D_{\perp}^{\prime} C_{1} U_{1} & 0 & 0 & 0 \\
-B_{2}^{\prime} U_{1} X_{1} & F V_{1} & 0 & 0
\end{array}\right]
$$

An alternative realization for $R(s)$ can be obtained via a state-space transformation

$$
T_{R}=\left[\begin{array}{cc}
I & -U_{1}^{\prime} V_{1} \\
0 & I
\end{array}\right]
$$

That is,

$$
R(s) \stackrel{s}{=}\left[\begin{array}{cc|cc}
-A_{F}^{\prime} & C_{1 F}^{\prime} C_{1} V_{1} & 0 & U_{1}^{\prime} H \\
0 & -A_{H}^{\prime} & V_{1}^{\prime} B_{1} \tilde{D}_{\perp}^{\prime} & -Y_{1} V_{1}^{\prime} C_{2}^{\prime} \\
\hline D_{\perp}^{\prime} C_{1} U_{1} & D_{\perp}^{\prime} C_{1} V_{1} & 0 & 0 \\
-B_{2}^{\prime} U_{1} X_{1} & D_{12}^{\prime} C_{1} V_{1} & 0 & 0
\end{array}\right]
$$

where

$$
\begin{aligned}
& A_{F}=X_{1} U_{1}^{\prime}\left(A-B_{2} F\right) U_{1} X_{1}^{-1} \\
& A_{H}=Y_{1}^{-1} V_{1}^{\prime}\left(A-H C_{2}\right) V_{1} Y_{1} \\
& B_{1 H}=Y_{1}^{-1} V_{1}^{\prime}\left(B_{1}-H D_{21}\right)=Y_{1}^{-1} V_{1}^{\prime} B_{1} \tilde{D}_{\perp}^{\prime} \tilde{D}_{\perp}-V_{1}^{\prime} C_{2}^{\prime} D_{21} \\
& C_{1 F}=\left(C_{1}-D_{12} F\right) U_{1} X_{1}^{-1}=D_{\perp} D_{\perp}^{\prime} C_{1} U_{1} X_{1}^{-1}-D_{12} B_{2}^{\prime} U_{1}
\end{aligned}
$$

and the Riccati equations for $X$ and $Y$ can be rewritten as

$$
\begin{aligned}
& A_{F}^{\prime} X_{1}^{-1}+X_{1}^{-1} A_{F}+C_{1 F}^{\prime} C_{1 F}=0, \\
& A_{H} Y_{1}^{-1}+Y_{1}^{-1} A_{H}^{\prime}+B_{1 H} B_{1 H}^{\prime}=0 .
\end{aligned}
$$

5.2. Necessary and sufficient conditions. A necessary condition for

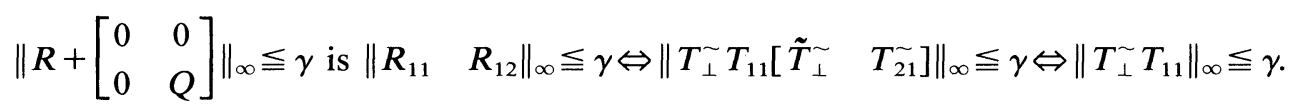

However,

$$
\begin{aligned}
T_{\perp}^{\sim} T_{11} & \stackrel{s}{=}\left[\begin{array}{c|c}
-\left(A-B_{2} F\right)^{\prime} & X B_{1} \\
\hline D_{\perp}^{\prime} C_{1} X^{\dagger} & 0
\end{array}\right] \\
& \stackrel{s}{=}\left[\begin{array}{c|c}
-A_{F}^{\prime} & U_{1}^{\prime} B_{1} \\
\hline D_{\perp}^{\prime} C_{1} U_{1} & 0
\end{array}\right]
\end{aligned}
$$

so that the corresponding spectral factorization Riccati equation to find $R_{13}$ (note that $\left.R_{13} R_{13}^{\sim}=\gamma^{2} I-\left[\begin{array}{ll}R_{11} & R_{12}\end{array}\right]\left[\begin{array}{ll}R_{11}^{\sim} & R_{12}^{\sim}\end{array}\right]^{\sim}=\gamma^{2} I-T_{\perp}^{\sim} T_{11} T_{11}^{\sim} T_{\perp}\right)$ has a solution $\hat{X} \geqq 0$ satisfying

$$
H_{\hat{X}}\left[\begin{array}{c}
I \\
\hat{X}
\end{array}\right]=\left[\begin{array}{c}
I \\
\hat{X}
\end{array}\right] T_{\hat{X}}, \quad \operatorname{Re} \lambda_{i}\left(T_{\hat{X}}\right) \leqq 0 \quad \forall i
$$

where

$$
H_{\hat{X}}=\left[\begin{array}{cc}
A_{F} & \gamma^{-2} U_{1}^{\prime} C_{1}^{\prime} D_{\perp} D_{\perp}^{\prime} C_{1} U_{1} \\
-U_{1}^{\prime} B_{1} B_{1}^{\prime} U_{1} & -A_{F}^{\prime}
\end{array}\right]
$$


Similarly, the condition $\left\|R_{11}^{\sim} \quad R_{21}^{\sim}\right\|_{\infty} \leqq \gamma$ implies that there exists $\hat{Y} \geqq 0$ such that

$$
H_{\hat{Y}}\left[\begin{array}{c}
I \\
\hat{Y}
\end{array}\right]=\left[\begin{array}{c}
I \\
\hat{Y}
\end{array}\right] T_{\hat{Y}}, \quad \operatorname{Re} \lambda_{i}\left(T_{\hat{Y}}\right) \leqq 0 \quad \forall i,
$$

where

$$
H_{\hat{Y}}=\left[\begin{array}{cc}
A_{H}^{\prime} & \gamma^{-2} V_{1}^{\prime} B_{1} \tilde{D}_{\perp}^{\prime} \tilde{D}_{\perp} B_{1}^{\prime} V_{1} \\
-V_{1}^{\prime} C_{1}^{\prime} C_{1} V_{1} & -A_{H}
\end{array}\right] .
$$

In order to apply the necessary and sufficient conditions of Theorem 4.2 , the solutions to the Riccati equations (3.51) and (3.52) (which determine $R_{13}$ and $R_{31}$ ) in $R(s)$ are required, and they are now given.

Lemma 5.3. For the realization of $R(s)$ defined in (5.46) the Riccati equations:

$$
\begin{gathered}
-P_{1} A_{R}^{\prime}-A_{R} P_{1}+B_{R 1} B_{R 1}^{\prime}+B_{R 2} B_{R 2}^{\prime}+\gamma^{-2} P_{1} C_{R 1}^{\prime} C_{R 1} P_{1}=0 \\
-Q_{1} A_{R}-A_{R}^{\prime} Q_{1}+C_{R 1}^{\prime} C_{R 1}+C_{R 2}^{\prime} C_{R 2}+\gamma^{-2} Q_{1} B_{R 1}^{\prime} B_{R 1} Q_{1}=0
\end{gathered}
$$

are satisfied for

$$
\begin{array}{cc}
P_{1}=\left[\begin{array}{cc}
\hat{X} & 0 \\
0 & Y_{1}
\end{array}\right], & Q_{1}=T_{R}^{\prime} Q_{0} T_{R} \\
T_{R}=\left[\begin{array}{cc}
I & -U_{1}^{\prime} V_{1} \\
0 & I
\end{array}\right], & Q_{0}=\left[\begin{array}{cc}
X_{1} & 0 \\
0 & \hat{Y}
\end{array}\right],
\end{array}
$$

and

$$
\begin{array}{ll}
\operatorname{Re} \lambda_{i}\left(A_{R}-\gamma^{-2} P_{1} C_{R 1}^{\prime} C_{R 1}\right) \geqq 0 & \forall i, \\
\operatorname{Re} \lambda_{i}\left(A_{R}-\gamma^{-2} B_{R 1} B_{R 1}^{\prime} Q_{1}\right) \geqq 0 & \forall i .
\end{array}
$$

Proof. $P_{1}$ is simply shown to satisfy (5.52) by (5.49) and (5.50) with antistability given by the stability of $T_{\hat{X}}$ and $A_{H}$. An analogous derivation applies to $Q_{0}$ for the alternative realization of $R$ in (5.47) and then $T_{R}^{\prime-1}$ relates $Q_{1}$ to $Q_{0}$.

Proof of Theorem 5.1. Theorem 4.2 and the above calculations give the necessary and sufficient conditions for controllers to exist to be that $\hat{X} \geqq 0$ and $\hat{Y} \geqq 0$ exist, and

$$
\lambda_{\max }\left(P_{1} Q_{1}\right) \leqq \gamma^{2} .
$$

To write this condition as that stated in the theorem, we write

$$
\begin{aligned}
\gamma^{2} I-P_{1} Q_{1} & =\left(\gamma^{2} T_{R}^{-1}-P_{1} T_{R}^{\prime} Q_{0}\right) T_{R} \\
& =\left[\begin{array}{cc}
\gamma I & 0 \\
0 & Y_{1}
\end{array}\right] \Pi(\gamma)\left[\begin{array}{cc}
X_{1} & 0 \\
0 & \gamma I
\end{array}\right] T_{R}
\end{aligned}
$$

in which

$$
\Pi(\gamma)=\left[\begin{array}{cc}
\gamma X_{1}^{-1}-\gamma^{-1} \hat{X} & U_{1}^{\prime} V_{1} \\
V_{1}^{\prime} U_{1} & \gamma Y_{1}^{-1}-\gamma^{-1} \hat{Y}
\end{array}\right] .
$$

Since $P_{1}$ and $Q_{1}$ are both monotonically decreasing functions of $\gamma$ [51], $\lambda_{\max }\left(P_{1} Q_{1}\right)$ is also a monotonically decreasing function of $\gamma$, and so $\lambda_{\max }\left(P_{1} Q_{1}\right) \leqq \gamma^{2}$ if and only if $\Pi(\gamma) \geqq 0$. 
This condition now needs to be written in terms of $X_{\infty 1}, X_{\infty 2}, Y_{\infty 1}$, and $Y_{\infty 2}$. The Riccati equations for $X_{1}(5.48)$ give, as in [11, $\S$ VII.C], that

$$
\left[\begin{array}{cc}
X_{1}^{-1} & -\gamma^{-2} I \\
I & 0
\end{array}\right] H_{\hat{x}}\left[\begin{array}{cc}
0 & I \\
-\gamma^{-2} I & \gamma^{2} X_{1}^{-1}
\end{array}\right]=\left[\begin{array}{cc}
U_{1}^{\prime} & 0 \\
0 & U_{1}^{\prime}
\end{array}\right] H_{\infty}\left[\begin{array}{cc}
U_{1} & 0 \\
0 & U_{1}
\end{array}\right] .
$$

Hence

$$
\begin{aligned}
{\left[\begin{array}{cc}
U_{1}^{\prime} & 0 \\
O & U_{1}^{\prime}
\end{array}\right] H_{\infty}\left[\begin{array}{cc}
U_{1} & 0 \\
0 & U_{1}
\end{array}\right]\left[\begin{array}{cc}
X_{1}^{-1} & -\gamma^{-2} I \\
I & 0
\end{array}\right]\left[\begin{array}{c}
I \\
\hat{X}
\end{array}\right] } & =\left[\begin{array}{cc}
X_{1}^{-1} & -\gamma^{-2} I \\
I & 0
\end{array}\right]\left[\begin{array}{c}
I \\
\hat{X}
\end{array}\right] T_{\hat{X}} \\
& =\left[\begin{array}{c}
X_{1}^{-1}-\gamma^{-2} \hat{X} \\
I
\end{array}\right] T_{\hat{x}}
\end{aligned}
$$

and exploiting the structure of $U^{\prime}\left(A-B_{2} D_{12}^{\prime} C_{1}\right) U$ and $U^{\prime} C_{1}^{\prime} D_{\perp}$ give

$$
H_{\infty}\left[\begin{array}{c}
X_{\infty 1} \\
X_{\infty 2}
\end{array}\right]=\left[\begin{array}{c}
X_{\infty 1} \\
X_{\infty 2}
\end{array}\right] T_{x}
$$

where

$$
\begin{gathered}
X_{\infty 1}=U_{1}\left(X_{1}^{-1}-\gamma^{-2} \hat{X}\right) U_{1}^{\prime}+U_{2} U_{2}^{\prime}, \\
X_{\infty 2}=U_{1} U_{1}^{\prime}
\end{gathered}
$$

satisfy

$$
X_{\infty 1}^{\prime} X_{\infty 2}=X_{\infty 2}^{\prime} X_{\infty 1}
$$

and all such matrices are given by $\left[\begin{array}{l}X_{\infty}^{\infty 1} \\ X_{\infty 2}\end{array} S\right.$ for a nonsingular $S$. (This comes from the strict stability of $U_{2}^{\prime}\left(A-B_{2} D_{12}^{\prime} C_{1}\right) U_{2}$ and the uniqueness of $\hat{X}$.) Furthermore, exploiting the structure of $\left[\begin{array}{cc}U^{\prime} & 0 \\ 0 & U^{\prime}\end{array}\right] H_{\infty}\left[\begin{array}{cc}U & 0 \\ 0 & U\end{array}\right]$ shows that if $X_{\infty 1}^{\prime} X_{\infty 22}=X_{\infty 2}^{\prime} X_{\infty 1}$ then $\hat{X}=\hat{X}^{\prime}$ exists. An analogous argument applies to $Y_{\infty 1}, Y_{\infty 2}$, and $\hat{Y}$. The final condition $\Pi(\gamma) \geqq 0$ is equivalent to

$$
\begin{aligned}
0 & \leqq\left[\begin{array}{cc}
U_{1} & 0 \\
0 & V_{1}
\end{array}\right] \Pi(\gamma)\left[\begin{array}{cc}
U_{1}^{\prime} & 0 \\
0 & V_{1}^{\prime}
\end{array}\right] \\
& =\gamma\left[\begin{array}{cc}
X_{\infty 2}^{\prime} X_{\infty 1} & \gamma^{-1} X_{\infty 2}^{\prime} Y_{\infty 2} \\
\gamma^{-1} Y_{\infty 2}^{\prime} X_{\infty 22} & Y_{\infty 2}^{\prime} Y_{\infty 1}
\end{array}\right] .
\end{aligned}
$$

5.3. Characterization of all solutions. Theorem 4.4 characterizes all optimal solutions to the model matching problem and Theorem 5.2 claims to do the same for the feedback control problem. A derivation of the formula in Theorem 5.2 can be obtained by applying Theorem 4.4 to generate all $Q(s)$ 's, and then substituting into $K_{0}(s)$ to generate all $K(s)$ 's. Finally, all the nonminimal modes are removed from this parameterization. In the rest of this section we assume, without loss of generality, that $\gamma=1$ for simplicity.

Applying Theorem 4.2 to the realization of $R$ given in (5.46) gives

$$
\begin{aligned}
{\left[\begin{array}{ll}
Q_{22} & Q_{24} \\
Q_{42} & Q_{44}
\end{array}\right]=\left[\begin{array}{ll}
0 & I \\
I & 0
\end{array}\right]+\left[\begin{array}{l}
C_{R 2} P_{1} \\
-B_{R 2}^{\prime}
\end{array}\right] } \\
\\
\cdot\left(\left(I-Q_{1} P_{1}\right) s-\left(-A_{R}^{\prime}-Q_{1} A_{R} P_{1}+Q_{1} B_{R 1} B_{R 1}^{\prime}+C_{R 1}^{\prime} C_{R 1} P_{1}\right)\right)^{-1} \\
\cdot\left[Q_{1} B_{R 2}-C_{R 2}^{\prime}\right] .
\end{aligned}
$$

Recall from (5.53) that

$$
\left(I-Q_{1} P_{1}\right)=T_{R}^{\prime}\left[\begin{array}{cc}
X_{1} & 0 \\
0 & I
\end{array}\right] \Pi\left[\begin{array}{cc}
I & 0 \\
0 & Y_{1}
\end{array}\right]
$$


in which $\Pi=\Pi(1)$. Changing coordinates gives

$$
\left[\begin{array}{ll}
Q_{22} & Q_{24} \\
Q_{42} & Q_{44}
\end{array}\right]=\left[\begin{array}{ll}
0 & I \\
I & 0
\end{array}\right]+\left[\begin{array}{l}
\hat{C}_{01} \\
\hat{C}_{02}
\end{array}\right]\left(s \Pi-\hat{A}_{0}\right)^{-1}\left[\begin{array}{ll}
\hat{B}_{01} & \hat{B}_{02}
\end{array}\right]
$$

$$
\hat{A}_{0}=\left[\begin{array}{cc}
X_{1}^{-1} & 0 \\
0 & I
\end{array}\right]\left(-T_{R}^{\prime-1} A_{R}^{\prime}-Q_{0} T_{R} A_{R} P_{1}+Q_{0} T_{R} B_{R 1} B_{R 1}^{\prime}+T_{R}^{\prime-1} C_{R 1}^{\prime} C_{R 1} P_{1}\right)
$$

$$
\left[\begin{array}{cc}
I & 0 \\
0 & Y_{1}^{-1}
\end{array}\right]
$$

$$
\begin{aligned}
& {\left[\begin{array}{l}
\hat{C}_{01} \\
\hat{C}_{02}
\end{array}\right]=\left[\begin{array}{c}
C_{R 2} P_{1} \\
-B_{R 2}^{\prime}
\end{array}\right]\left[\begin{array}{cc}
I & 0 \\
0 & Y_{1}^{-1}
\end{array}\right]=\left[\begin{array}{cc}
-B_{2}^{\prime} U_{1} X_{1} \hat{X} & F V_{1} \\
-D_{21} B_{1}^{\prime} U_{1} & C_{2} V_{1}
\end{array}\right],} \\
& {\left[\begin{array}{ll}
\hat{B}_{01} & \hat{B}_{02}
\end{array}\right]=\left[\begin{array}{cc}
X_{1}^{-1} & 0 \\
0 & I
\end{array}\right]\left[\begin{array}{ll}
Q_{0} T_{R} B_{R 2} & -T_{R}^{\prime-1} C_{R 2}^{\prime}
\end{array}\right]} \\
& =\left[\begin{array}{cc}
U_{1}^{\prime} H & U_{1}^{\prime} B_{2} \\
-\hat{Y} Y_{1} V_{1}^{\prime} C_{2}^{\prime} & -V_{1}^{\prime} C_{1}^{\prime} D_{12}
\end{array}\right] \text {, } \\
& \hat{A}_{011}=X_{1}^{-1} A_{F}+A_{F}^{\prime} \hat{X}+X_{1}^{-1} U_{1}^{\prime} C_{1}^{\prime} D_{\perp} D_{\perp}^{\prime} C_{1} U_{1} \hat{X} \\
& =X_{1}^{-1} A_{F}-X_{1}^{-1} A_{F} X_{1} \hat{X}-U_{1}^{\prime} B_{2} B_{2}^{\prime} U_{1} X_{1} \hat{X} \\
& \hat{A}_{012}=-\left(U_{1}^{\prime} B_{1} B_{1 H}^{\prime}+U_{1}^{\prime} V_{1} A_{H}^{\prime}\right)=-U_{1}^{\prime}\left(B_{1}\left(B_{1}-H D_{21}\right)^{\prime}+Y\left(A-H C_{2}\right)^{\prime}\right) V_{1} Y_{1}^{-1} \\
& =U_{1}^{\prime} A Y V_{1} Y_{1}^{-1}=U_{1}^{\prime} A V_{1} \text {, } \\
& \hat{A}_{021}=-\left(B_{1 H} B_{1}^{\prime} U_{1}-V_{1}^{\prime} U_{1} A_{F}\right)+\hat{Y} V_{1}^{\prime} B_{1} \tilde{D}_{\perp}^{\prime} \tilde{D}_{\perp} B_{1}^{\prime} U_{1}+V_{1}^{\prime} C_{1}^{\prime} D_{\perp} D_{\perp}^{\prime} C_{1} U_{1} \hat{X} \\
& =\left(\hat{Y}-Y_{1}^{-1}\right) V_{1}^{\prime} B_{1} \tilde{D}_{\perp}^{\prime} \tilde{D}_{\perp} B_{1}^{\prime} U_{1}+V_{1}^{\prime} C_{1}^{\prime} D_{\perp} D_{\perp}^{\prime} C_{1} U_{1}\left(\hat{X}-X_{1}^{-1}\right) \\
& -V_{1}^{\prime}\left(A-B_{2} D_{12}^{\prime} C_{1}-B_{1} D_{21}^{\prime} C_{2}\right)^{\prime} U_{1} \\
& \hat{A}_{022}=A_{H} Y_{1}^{-1}+\hat{Y} A_{H}^{\prime}+\hat{Y} V_{1}^{\prime} B_{1} \tilde{D}_{\perp}^{\prime} \tilde{D}_{\perp} B_{1}^{\prime} V_{1} Y_{1}^{-1} \\
& =A_{H} Y_{1}^{-1}+\hat{Y} Y_{1}\left(-A_{H} Y_{1}^{-1}-V_{1}^{\prime} C_{2}^{\prime} C_{2} V_{1}\right) \\
& =\left(I-\hat{Y} Y_{1}\right) A_{H} Y_{1}^{-1}-\hat{Y} Y_{1} V_{1}^{\prime} C_{2}^{\prime} C_{2} V_{1} \text {. }
\end{aligned}
$$

The characterization of all $K(s)$ is given by

$$
\begin{aligned}
K(s) & =F_{l}\left(K_{0}, F_{l}\left(\left[\begin{array}{ll}
Q_{22} & Q_{24} \\
Q_{42} & Q_{44}
\end{array}\right], U\right)\right) \\
& =F_{l}(\mathscr{K}, U)
\end{aligned}
$$

where

$$
\mathscr{K}=F_{l}\left\{\left[\begin{array}{cccc}
K_{011} & 0 & K_{012} & 0 \\
0 & 0 & 0 & I \\
K_{021} & 0 & K_{022} & 0 \\
0 & I & 0 & 0
\end{array}\right],\left[\begin{array}{ll}
Q_{22} & Q_{24} \\
Q_{42} & Q_{44}
\end{array}\right]\right\}
$$

Substituting for the realization gives that

$$
\mathscr{K}(s)=\left[\begin{array}{ll}
0 & I \\
I & 0
\end{array}\right]+\hat{C}_{1}\left(s \hat{E}_{1}-\hat{A}_{1}\right)^{-1} \hat{B}_{1}
$$


where

$$
\begin{aligned}
& \hat{E}_{1}=\left[\begin{array}{ll}
I & 0 \\
0 & \Pi
\end{array}\right], \\
& \hat{A}_{1}=\left[\begin{array}{cc}
A-B_{2} F-H C_{2} & B_{2} \hat{C}_{01} \\
-\hat{B}_{01} C_{2} & \hat{A}_{0}
\end{array}\right], \\
& \hat{B}_{1}=\left[\begin{array}{cc}
H & B_{2} \\
\hat{B}_{01} & \hat{B}_{02}
\end{array}\right], \\
& \hat{C}_{1}=\left[\begin{array}{ll}
-F & \hat{C}_{01} \\
-C_{2} & \hat{C}_{02}
\end{array}\right] .
\end{aligned}
$$

This realization contains $\operatorname{rank}(X)$ uncontrollable modes and $\operatorname{rank}(Y)$ unobservable modes and these are exhibited by the following transformation. First, define

$$
\begin{aligned}
& X_{\infty 11}=X_{1}^{-1}-\hat{X}, \quad Y_{\infty 11}=Y_{1}^{-1}-\hat{Y} \\
& T_{l}=\left[\begin{array}{ccc}
V^{\prime} & 0 & 0 \\
-U_{1}^{\prime} & I & 0 \\
-Y_{\infty 11}^{\prime} V_{1}^{\prime} & 0 & I
\end{array}\right], \quad T_{r}=\left[\begin{array}{ccc}
U & U_{1} X_{\infty 11} & V_{1} \\
0 & I & 0 \\
0 & 0 & I
\end{array}\right] \text {, } \\
& \hat{E}_{2}=T_{l} \hat{E}_{1} T_{r}=\left[\begin{array}{ccc}
V^{\prime} U & V^{\prime} U_{1} X_{\infty 11} & {\left[\begin{array}{l}
I \\
0
\end{array}\right]} \\
{\left[\begin{array}{cc}
-I & 0
\end{array}\right]} & 0 & 0 \\
-Y_{\infty 11} V_{1}^{\prime} U & V_{1}^{\prime} U_{1}-Y_{\infty 11} V_{1}^{\prime} U_{1} X_{\infty 11} & 0
\end{array}\right] \text {, } \\
& \hat{A}_{2}=T_{l} \hat{A}_{1} T_{r}=\left[\begin{array}{lll}
\hat{A}_{211} & \hat{A}_{212} & \hat{A}_{213} \\
\hat{A}_{221} & \hat{A}_{222} & \hat{A}_{223} \\
\hat{A}_{231} & \hat{A}_{232} & \hat{A}_{233}
\end{array}\right] \text {, } \\
& \hat{B}_{2}=T_{l} \hat{B}_{1}=\left[\begin{array}{c}
\hat{B}_{21} \\
\hat{B}_{22} \\
\hat{B}_{23}
\end{array}\right], \quad \hat{C}_{2}=\hat{C}_{1} T_{r}=\left[\begin{array}{lll}
\hat{C}_{21} & \hat{C}_{22} & \hat{C}_{23}
\end{array}\right] \text {, } \\
& \hat{B}_{22}=0 \text { by (5.56), } \\
& \hat{C}_{23}=0 \text { by (5.57), }
\end{aligned}
$$

$\left[\begin{array}{lll}\hat{A}_{221} & \hat{A}_{222} & \hat{A}_{223}\end{array}\right]=-U_{1}^{\prime}\left[\begin{array}{lll}A-B_{2} F-H C_{2} & -B_{2} B_{2}^{\prime} U_{1} X_{1} \hat{X} & B_{2} F V_{1}\end{array}\right] T_{r}$ $+\left[\begin{array}{lll}-U_{1}^{\prime} H C_{2} & \hat{A}_{011} & \hat{A}_{012}\end{array}\right] T_{r}$ $=\left[\begin{array}{lll}-U_{1}^{\prime}\left(A-B_{2} F\right) & X_{1}^{-1} A_{F} X_{1} X_{\infty 11} & \hat{A}_{012}-U_{1}^{\prime} B_{2} F V_{1}\end{array}\right] T_{r}$

$$
\Rightarrow \hat{A}_{221}=\left[\begin{array}{ll}
-U_{1}^{\prime}\left(A-B_{2} F\right) U_{1} & 0
\end{array}\right], \quad \hat{A}_{222}=0, \quad \hat{A}_{223}=0
$$

Hence the first rank $(X)$ states are uncontrollable. Furthermore,

$$
\begin{aligned}
& \hat{A}_{213}=V^{\prime}\left(A-B_{2} F-H C_{2}\right) V_{1}+V^{\prime} B_{2} F V_{1}=\left[\begin{array}{c}
V_{1}^{\prime}\left(A-H C_{2}\right) V_{1} \\
0
\end{array}\right], \\
& {\left[\begin{array}{lll}
\hat{A}_{231} & \hat{A}_{232} & \hat{A}_{233}
\end{array}\right]=-Y_{\infty 11} V_{1}^{\prime}\left[A-B_{2} F-H C_{2} \quad-B_{2} B_{2}^{\prime} U_{1} X_{1} \hat{X} \quad B_{2} F V_{1}\right] T_{r}} \\
& +\left[\begin{array}{lll}
\hat{Y} Y_{1} V_{1}^{\prime} C_{2}^{\prime} C_{2} & \hat{A}_{021} & \hat{A}_{022}
\end{array}\right] T_{r}
\end{aligned}
$$


$\Rightarrow \hat{A}_{233}=0$ and the last $\operatorname{rank}(Y)$ states are unobservable. A reduced state model can hence be obtained and its realization transformed as follows:

$$
\hat{E}_{3}=\left[\begin{array}{ll}
V_{2} & -V_{1}
\end{array}\right]\left[\begin{array}{cc}
V_{2}^{\prime} U_{2} & V_{2}^{\prime} U_{1} X_{\infty 11} \\
-Y_{\infty 11} V_{1}^{\prime} U_{2} & V_{1}^{\prime} U_{1}-Y_{\infty 11} V_{1}^{\prime} U_{1} X_{\infty 11}
\end{array}\right]\left[\begin{array}{c}
U_{2}^{\prime} \\
U_{1}^{\prime}
\end{array}\right]
$$

without loss of generality (see proof of Theorem 5.1) we can assume

$$
\begin{aligned}
X_{\infty 1} & =U\left[\begin{array}{cc}
X_{\infty 11} & 0 \\
0 & I
\end{array}\right] U^{\prime}, & X_{\infty 2} & =U_{1} U_{1}^{\prime}, \\
Y_{\infty 1} & =V\left[\begin{array}{cc}
Y_{\infty 11} & 0 \\
0 & I
\end{array}\right] V^{\prime}, & Y_{\infty 2} & =V_{1} V_{1}^{\prime}
\end{aligned}
$$

and hence

$$
\begin{aligned}
& \hat{E}_{3}=Y_{\infty 1}^{\prime} X_{\infty 1}-Y_{\infty 22}^{\prime} X_{\infty 2}=E_{k} \text {, } \\
& \hat{B}_{3}=\left[\begin{array}{ll}
B_{k 1} & B_{k 2}
\end{array}\right]=\left[\begin{array}{ll}
V_{2} & -V_{1}
\end{array}\right]\left[\begin{array}{cc}
V_{2}^{\prime} & 0 \\
-Y_{\infty 11}^{\prime} V_{1}^{\prime} & I
\end{array}\right]\left[\begin{array}{cc}
H & B_{2} \\
-\hat{Y} Y_{1} V_{1}^{\prime} C_{2}^{\prime} & -V_{1}^{\prime} C_{1}^{\prime} D_{12}
\end{array}\right] \\
& =\left[\begin{array}{ll}
Y_{\infty 1}^{\prime} & -V_{1}
\end{array}\right]\left[\begin{array}{cc}
H & B_{2} \\
-\hat{Y} Y_{1} V_{1}^{\prime} C_{2}^{\prime} & -V_{1}^{\prime} C_{1}^{\prime} D_{12}
\end{array}\right] \\
& =\left[Y_{\infty 1}^{\prime}\left(Y C_{2}^{\prime}+B_{1} D_{21}^{\prime}\right)+V_{1}\left(I-Y_{\infty 11} Y_{1}\right) V_{1}^{\prime} C_{2}^{\prime}\right. \text {, } \\
& \left.Y_{\infty 1}^{\prime} B_{2}+V_{1} V_{1}^{\prime} C_{1}^{\prime} D_{12}\right] \\
& =\left[\begin{array}{ll}
Y_{\infty 2}^{\prime} C_{2}^{\prime}+Y_{\infty 1}^{\prime} B_{1} D_{21}^{\prime} \quad Y_{\infty 1}^{\prime} B_{2}+Y_{\infty 2}^{\prime} C_{1}^{\prime} D_{12}
\end{array}\right] \text {, } \\
& \hat{C}_{3}=\left[\begin{array}{l}
C_{k 1} \\
C_{k 2}
\end{array}\right]=\left[\begin{array}{cc}
-F & -B_{2}^{\prime} U_{1} X_{1} \hat{X} \\
-C_{2} & -D_{21} B_{1}^{\prime} U_{1}
\end{array}\right]\left[\begin{array}{cc}
U_{2} & U_{1} X_{\infty 11} \\
0 & I
\end{array}\right]\left[\begin{array}{l}
U_{2}^{\prime} \\
U_{1}^{\prime}
\end{array}\right] \\
& =-\left[\begin{array}{cc}
F & B_{2}^{\prime} U_{1} X_{1} \hat{X} \\
C_{2} & D_{21} B_{1}^{\prime} U_{1}
\end{array}\right]\left[\begin{array}{c}
X_{\infty 1} \\
U_{1}^{\prime}
\end{array}\right] \\
& =-\left[\begin{array}{l}
D_{12}^{\prime} C_{1} X_{\infty 1}+B_{2}^{\prime} X_{\infty 2} \\
C_{2} X_{\infty 1}+D_{21} B_{1}^{\prime} X_{\infty 2}
\end{array}\right] \text {, } \\
& \hat{A}_{3}=\left[\begin{array}{ll}
Y_{\infty 1}^{\prime} & -V_{1}
\end{array}\right]\left[\begin{array}{cc}
A-B_{2} F-H C_{2} & -B_{2} B_{2}^{\prime} U_{1} X_{1} \hat{X} \\
\hat{Y} Y_{1} V_{1}^{\prime} C_{2}^{\prime} C_{2} & \hat{A}_{021}
\end{array}\right]\left[\begin{array}{c}
X_{\infty 1} \\
U_{1}^{\prime}
\end{array}\right] \\
& =\left[\begin{array}{ll}
Y_{\infty 1}^{\prime} & -Y_{\infty 2}^{\prime}
\end{array}\right]\left\{H_{\infty}+\left[\begin{array}{c}
-B_{1} D_{21}^{\prime} \\
C_{2}^{\prime}
\end{array}\right]\left[\begin{array}{ll}
C_{2} & D_{21} B_{1}^{\prime}
\end{array}\right]+\left[\begin{array}{ll}
L_{11} & L_{12} \\
L_{21} & L_{22}
\end{array}\right]\right\}\left[\begin{array}{l}
X_{\infty 1} \\
X_{\infty 2}
\end{array}\right]
\end{aligned}
$$

where

$$
\begin{gathered}
L_{11}=-B_{2} B_{2}^{\prime} X-Y C_{2}^{\prime} C_{2}, \\
L_{12}=B_{2} B_{2}^{\prime}\left(I-U_{1} X_{1} \hat{X} U_{1}^{\prime}\right)-B_{1} \tilde{D}_{\perp}^{\prime} \tilde{D}_{\perp} B_{1}^{\prime}, \\
L_{21}=-\left(I-V_{1} \hat{Y} Y_{1} V_{1}^{\prime}\right) C_{2}^{\prime} C_{2}+C_{1}^{\prime} D_{\perp} D_{\perp}^{\prime} C_{1}, \\
L_{22}=\left(A-B_{2} D_{12}^{\prime} C_{1}-B_{1} D_{21}^{\prime} C_{2}\right)^{\prime}+V_{1} \hat{A}_{021} U_{1}^{\prime}, \\
V_{1}^{\prime} L_{22} U_{1}=\left(\hat{Y}-Y_{1}^{-1}\right) V_{1}^{\prime} B_{1} \tilde{D}_{\perp}^{\prime} \tilde{D}_{\perp} B_{1}^{\prime} U_{1}+V_{1}^{\prime} C_{1}^{\prime} D_{\perp} D_{\perp}^{\prime} C_{1} U_{1}\left(\hat{X}-X_{1}^{-1}\right) .
\end{gathered}
$$


The equations $X X_{\infty 1}+U_{1} X_{1} \hat{X} U_{1}^{\prime}=U_{1} U_{1}^{\prime}$ and $Y_{\infty 1} Y+V_{1} \hat{Y} Y_{1} V_{1}^{\prime}=V_{1} V_{1}^{\prime}$ and direct substitution gives

$$
\left[\begin{array}{ll}
Y_{\infty 1} & -Y_{\infty 2}
\end{array}\right]\left[\begin{array}{ll}
L_{11} & L_{12} \\
L_{21} & L_{22}
\end{array}\right]\left[\begin{array}{l}
X_{\infty 1} \\
X_{\infty 2}
\end{array}\right]=0
$$

and finally $\hat{A}_{3}=A_{k}$ since

$$
H_{\infty}\left[\begin{array}{l}
X_{\infty 1} \\
X_{\infty 2}
\end{array}\right]=\left[\begin{array}{l}
X_{\infty 1} \\
X_{\infty 2}
\end{array}\right] T_{x}
$$

5.4. Removing assumption (v). In this section we show how we might tackle problems in which $D_{11} \neq 0$ and/or $D_{22} \neq 0$. The idea is to show that assumption (v) results in no loss of generality, and that the original problem with $D_{11} \neq 0$ and $D_{22} \neq 0$ may be replaced with an equivalent problem in which $\hat{D}_{11}=0$ and $\hat{D}_{22}=0$. This observation is particularly useful in theoretical work requiring state-space calculations, since, as we will soon show, the case $D_{11} \neq 0$ leads to controllers with an unwieldy number of terms.

Step 1. The purpose of this step is to solve the four block problem at infinity. Suppose $F_{\infty}$ is a constant feedback to be defined, and suppose also that $P(s)$ is as given in (5.1). Then for any such $F_{\infty}$

$$
\begin{aligned}
\bar{P}(s) & =F_{l}\left(\left[\begin{array}{ll|l}
P_{11} & P_{12} & P_{12} \\
P_{21} & P_{22} & P_{22} \\
\hline P_{21} & P_{22} & P_{22}
\end{array}\right], F_{\infty}\right) \\
& =\left[\begin{array}{c|cc}
\bar{A} & \bar{B}_{1} & \bar{B}_{2} \\
\hline \bar{C}_{1} & \bar{D}_{11} & \bar{D}_{12} \\
\bar{C}_{2} & \bar{D}_{21} & \bar{D}_{22}
\end{array}\right] \\
& =\left[\begin{array}{c|cc}
A+B_{2} F_{\infty}\left(I-D_{22} F_{\infty}\right)^{-1} C_{2} & B_{1}+B_{2} F_{\infty}\left(I-D_{22} F_{\infty}\right)^{-1} D_{21} & B_{2}\left(I-F_{\infty} D_{22}\right)^{-1} \\
\hline C_{1}+D_{12} F_{\infty}\left(I-D_{22} F_{\infty}\right)^{-1} C_{2} & D_{11}+D_{12} F_{\infty}\left(I-D_{22} F_{\infty}\right)^{-1} D_{21} & D_{12}\left(I-F_{\infty} D_{22}\right)^{-1} \\
\left(I-D_{22} F_{\infty}\right)^{-1} C_{2} & \left(I-D_{22} F_{\infty}\right)^{-1} D_{21} & \left(I-D_{22} F_{\infty}\right)^{-1} D_{22}
\end{array}\right] .
\end{aligned}
$$

To find an $F_{\infty}$ that solves the problem at infinity, we define $Q_{\infty}=F_{\infty}\left(I-D_{22} F_{\infty}\right)^{-1}$ and apply Parrott's result [39], which states that

$$
Q_{\infty}=-D_{12}^{\prime}\left(D_{11}+D_{11} \tilde{D}_{\perp}^{\prime}\left(\gamma^{2} I-\left(D_{\perp}^{\prime} D_{11} \tilde{D}_{\perp}^{\prime}\right)^{\prime}\left(D_{\perp}^{\prime} D_{11} \tilde{D}_{\perp}^{\prime}\right)\right)^{-1}\left(D_{\perp}^{\prime} D_{11} \tilde{D}_{\perp}^{\prime}\right)^{\prime} D_{\perp}^{\prime} D_{11}\right) D_{21}^{\prime}
$$

solves the problem

$$
\left\|\left[\begin{array}{cc}
D_{\perp}^{\prime} D_{11} \tilde{D}_{\perp}^{\prime} & D_{\perp}^{\prime} D_{11} D_{21}^{\prime} \\
D_{12}^{\prime} D_{11} \tilde{D}_{\perp}^{\prime} & D_{12}^{\prime} D_{11} D_{21}^{\prime}+Q_{\infty}
\end{array}\right]\right\|_{2}<\gamma .
$$

(A solution exists if and only if $\gamma>\gamma_{p}=\max \left\{\left\|D_{\perp}^{\prime} D_{11}\right\|,\left\|D_{11} \tilde{D}_{\perp}^{\prime}\right\|\right\}$ ). Back substitution gives

$$
F_{\infty}=\left(I+Q_{\infty} D_{22}\right)^{-1} Q_{\infty}
$$

in which the existence of the inverse is assumed. There are two points to note:

$$
\left\|\bar{D}_{11}\right\|_{2}<\gamma \text {. }
$$

(2) $I-D_{22} F_{\infty}=\left(I+D_{22} Q_{\infty}\right)^{-1}$ which shows that the existence of $\left(I+D_{22} Q_{\infty}\right)^{-1} \Rightarrow$ the existence of $\left(I-D_{22} F_{\infty}\right)^{-1}$. 
Step 2. Here we select an orthogonal $\Theta$-matrix in Fig. 1 such that $\left(F_{l}\left(\Theta, \bar{P}_{11}(s)\right)(\infty)\right)_{11}=0$. Note that

$$
\bar{D}_{11}:=D_{11}+D_{12} Q_{\infty} D_{21}
$$

and define

$$
\left[\begin{array}{ll}
\Theta_{11} & \Theta_{12} \\
\Theta_{21} & \Theta_{22}
\end{array}\right]=\gamma^{-1}\left[\begin{array}{cc}
\gamma^{-1} \bar{D}_{11} & \left(I-\gamma^{-2} \bar{D}_{11} \bar{D}_{11}^{\prime}\right)^{1 / 2} \\
-\left(I-\gamma^{-2} \bar{D}_{11}^{\prime} \bar{D}_{11}\right)^{1 / 2} & \gamma^{-1} \bar{D}_{11}^{\prime}
\end{array}\right],
$$

which satisfies $\Theta \Theta^{\prime}=\gamma^{-2} I$ for all $\gamma \geqq \gamma_{p}$. By direct computation

$$
\begin{aligned}
\hat{P}(s) & =F_{l}\left(\left[\begin{array}{cc|cc}
\Theta_{11} & 0 & \Theta_{12} & 0 \\
0 & 0 & 0 & I \\
\hline \Theta_{21} & 0 & \Theta_{22} & 0 \\
0 & I & 0 & 0
\end{array}\right], \bar{P}(s)\right)=\left[\begin{array}{c|cc}
\hat{A} & \hat{B}_{1} & \hat{B}_{2} \\
\hline \hat{C}_{1} & 0 & \hat{D}_{12} \\
\hat{C}_{2} & \hat{D}_{21} & \hat{D}_{22}
\end{array}\right] \\
& s\left[\begin{array}{ccc}
\bar{A}+\bar{B}_{1} \Theta_{22}\left(I-\bar{D}_{11} \Theta_{22}\right)^{-1} \bar{C}_{1} & \bar{B}_{1}\left(I-\Theta_{22} \bar{D}_{11}\right)^{-1} \Theta_{21} & \bar{B}_{2}+\bar{B}_{1} \Theta_{22}\left(I-\bar{D}_{11} \Theta_{22}\right)^{-1} \bar{D}_{12} \\
\hline \Theta_{12}\left(I-\bar{D}_{11} \Theta_{22}\right)^{-1} \bar{C}_{1} & 0 & \Theta_{12}\left(I-\bar{D}_{11} \Theta_{22}\right)^{-1} \bar{D}_{12} \\
\bar{C}_{2}+\bar{D}_{21} \Theta_{22}\left(I-\bar{D}_{11} \Theta_{22}\right)^{-1} \bar{C}_{1} & \bar{D}_{21}\left(I-\Theta_{22} \bar{D}_{11}\right)^{-1} \Theta_{21} & \bar{D}_{22}+\bar{D}_{21} \Theta_{22}\left(I-\bar{D}_{11} \Theta_{22}\right)^{-1} \bar{D}_{12}
\end{array}\right],
\end{aligned}
$$

which has the required property that $\hat{D}_{11}=0$. It is an immediate consequence of a specialization of Redheffer's theorem that $\left\|F_{l}(\bar{P}, K)\right\|_{\infty} \leqq \gamma$ if and only if $\left\|F_{l}(\bar{P}, K)\right\|_{\infty} \leqq$ $\gamma^{-1}[30]$. A small gain argument shows that the internal stability property is preserved for all $\gamma>\gamma_{p}$.

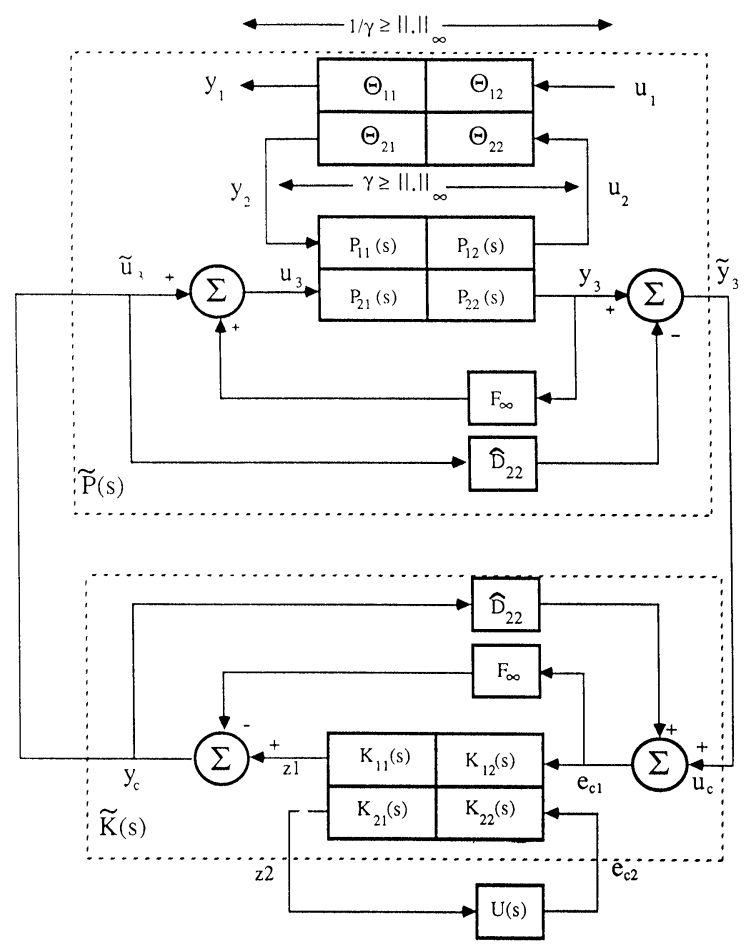

FIG. 1. Loop transformations. 
Step 3. Here we eliminate $\hat{D}_{22}$ by connecting $\left(\hat{D}_{22}-\hat{D}_{22}\right)$ in parallel with $\hat{P}_{22}(s)$ which is illustrated in Fig. 1. See also [20].

Step 4. Compute $\tilde{K}(s)$ using equations (5.11)-(5.18). (Note that $\hat{D}_{12}^{\prime} \hat{D}_{12} \neq I$ and $\hat{D}_{21} \hat{D}_{21}^{\prime} \neq I$ so another scaling is required.)

Step 5. Reverse the effects of $\hat{D}_{22}$ and $F_{\infty}$ to obtain a representation formula for all controllers. Yet another calculation verifies that

$$
\left[\begin{array}{ll}
K_{11} & K_{12} \\
K_{21} & K_{22}
\end{array}\right]=F_{l}\left\{\left[\begin{array}{cc|c}
F_{\infty} & \tilde{K}_{12} & -I \\
\tilde{K}_{21} & \tilde{K}_{22}-\tilde{K}_{21} \hat{D}_{22} \tilde{K}_{12} & \tilde{K}_{21} \hat{D}_{22} \\
\hline-I & \hat{D}_{22} \tilde{K}_{12} & -\hat{D}_{22}
\end{array}\right], \tilde{K}_{11}\right\} .
$$

Note that the effects of the scale introduced in Step 4 must now be reversed.

It remains to show that provided $\gamma$ is big enough, the realization for $P(s)$ in (5.1) satisfies (i)-(iv) if and only if the realization for $\hat{P}(s)$ given in $(5.70)$ satisfies these same assumptions. In our analysis below, we treat the transformation between $P(s)$ and $\bar{P}(s)$ first, and the transformation between $\bar{P}(s)$ and $\hat{P}(s)$ second.

LEMMA 5.4. Assumptions (i)-(iv) apply to the realization in (5.1) if and only if they apply to the realization in (5.70).

Proof. (i) Stabilizibility and detectability are invariant under output feedback. Assumption (ii) is immediate from (5.70). Assumption (iii) and (iv) follow from

$$
\begin{gathered}
{\left[\begin{array}{cc}
s I-\bar{A} & -\bar{B}_{2} \\
\bar{C}_{1} & \bar{D}_{12}
\end{array}\right]=\left[\begin{array}{cc}
s I-A & -B_{2} \\
C_{1} & D_{12}
\end{array}\right]\left[\begin{array}{cc}
I & 0 \\
F_{\infty}\left(I-D_{22} F_{\infty}\right)^{-1} C_{2} & \left(I-F_{\infty} D_{22}\right)^{-1}
\end{array}\right]} \\
{\left[\begin{array}{cc}
s I-\bar{A} & \bar{B}_{1} \\
-\bar{C}_{2} & \bar{D}_{21}
\end{array}\right]=\left[\begin{array}{cc}
I & B_{2} F_{\infty}\left(I-D_{22} F_{\infty}\right)^{-1} \\
0 & \left(I-D_{22} F_{\infty}\right)^{-1}
\end{array}\right]\left[\begin{array}{cc}
s I-A & B_{1} \\
-C_{2} & D_{21}
\end{array}\right] .}
\end{gathered}
$$

LEMMA 5.5. Suppose that an internally stabilizing controller exists such that $\left\|F_{l}(P, K)\right\|_{\infty} \leqq \gamma$. Then

(1) $\left(\hat{A}, \hat{B}_{2}, \hat{C}_{2}\right)$ is stabilizable and detectable.

(2) $\operatorname{rank}\left(D_{12}\right)=m \Leftrightarrow \operatorname{rank}\left(\hat{D}_{12}\right)=m$, and $\operatorname{rank}\left(D_{21}\right) \Leftrightarrow \operatorname{rank}\left(\hat{D}_{21}\right)=q$.

(3) $\operatorname{rank}\left(\left[\begin{array}{cc}j \omega I-A & B_{2} \\ -C_{1} & D_{12}\end{array}\right]\right)=n+m$ for all real $\omega$ if and only if $\operatorname{rank}\left(\left[\begin{array}{ccc}j \omega I-\hat{A} & \hat{B}_{2} \\ -\hat{C}_{1} & \hat{D}_{12}\end{array}\right]\right)=$ $n+m$ for all real $\omega$.

(4) $\operatorname{rank}\left(\left[\begin{array}{cc}j \omega I-A & -B_{1} \\ C_{2} & D_{21}\end{array}\right]\right)=n+q$ for all real $\omega$ if and only if $\operatorname{rank}\left(\left[\begin{array}{cc}j \omega I-\hat{A} & -\hat{B}_{1} \\ \hat{C}_{2} & \hat{D}_{21}\end{array}\right]\right)=$ $n+q$ for all real $\omega$.

Proof. (1) Since $\left\|\Theta_{22}\right\|_{2}<\gamma^{-1}$, it follows from a small gain argument that $F_{l}(\hat{P}, K)$ is internally stable. As $K$ is an internally stabilizing controller for $\hat{P}(s)$, it follows that $\left(\hat{A}, \hat{B}_{2}, \hat{C}_{2}\right)$ is stabilizable and detectable.

(2) This follows from the invertibility of $\Theta_{12}$ and $\Theta_{21},(5.70)$ and (5.71).

Parts (3) and (4) follow from Lemma 5.3 and the identities

$$
\begin{aligned}
& {\left[\begin{array}{cc}
s I-\hat{A} & \hat{B}_{2} \\
-\hat{C}_{1} & \hat{D}_{12}
\end{array}\right]=\left[\begin{array}{cc}
I & \bar{B}_{1} \Theta_{22}\left(I-\bar{D}_{11} \Theta_{22}\right)^{-1} \\
0 & \Theta_{12}\left(I-\bar{D}_{11} \Theta_{22}\right)^{-1}
\end{array}\right]\left[\begin{array}{cc}
s I-\bar{A} & \bar{B}_{2} \\
-\bar{C}_{1} & \bar{D}_{12}
\end{array}\right],} \\
& {\left[\begin{array}{cc}
s I-\hat{A} & -\hat{B}_{1} \\
\hat{C}_{2} & \hat{D}_{21}
\end{array}\right]=\left[\begin{array}{cc}
s I-\bar{A} & -\bar{B}_{1} \\
\bar{C}_{2} & \bar{D}_{21}
\end{array}\right]\left[\begin{array}{cc}
I & 0 \\
\Theta_{22}\left(I-\bar{D}_{11} \Theta_{22}\right)^{-1} \bar{C}_{1} & \left(I-D_{22} \Theta_{22}\right)^{-1} \Theta_{21}
\end{array}\right] \text {. }}
\end{aligned}
$$

If we need to calculate a controller generator in terms of the original plant description, it is possible to repeat calculations of the type given in the previous two sections (these have only been checked in detail by the present authors in the case where $X_{\infty}$ and $Y_{\infty}$ exist). In this event Step 2 is left out and we proceed as follows: Execute Step 1 as before; note that this step can be carried out if and only if $\max \left(\left\|D_{\perp}^{\prime} D_{11}\right\|_{2},\left\|D_{11} \tilde{D}_{\perp}^{\prime}\right\|_{2}\right)<\gamma$. After this step there holds $\left\|D_{11}\right\|_{2}<\gamma$. Execute Step 3 as before to remove $\bar{D}_{22}$. Execute Step 4 using the results of Theorem 5.1' and Theorem $5.2^{\prime}$ given below. End with Step 5 as before. 
THEOREM 5.1'. Suppose that $P(s)$ is given by (5.1), and that the assumptions (i)-(iv) are satisfied with $D_{22}=0$ and $\left\|D_{11}\right\|_{2}<\gamma$. Then internally stabilizing controllers exist for $\gamma>0$ with $\left\|F_{l}(P, K)\right\|_{\infty} \leqq \gamma$ if and only if

(i) Condition (i) of Theorem 5.1 is satisfied with (5.5) replaced by

$$
H_{\infty}=\left[\begin{array}{cc}
H_{\infty 11} & H_{\infty 12} \\
H_{\infty 21} & -H_{\infty 11}^{\prime}
\end{array}\right]
$$

in which

$$
\begin{aligned}
& H_{\infty 11}=A+\left(B_{1} D_{11}^{\prime} D_{\perp} D_{\perp}^{\prime}-\gamma^{2} B_{2} D_{12}^{\prime}\right)\left(\gamma^{2} I-D_{11} D_{11}^{\prime} D_{\perp} D_{\perp}^{\prime}\right)^{-1} C_{1} \\
& H_{\infty 21}=-C_{1}^{\prime} D_{\perp}\left(I-\gamma^{-2} D_{\perp}^{\prime} D_{11} D_{11}^{\prime} D_{\perp}\right)^{-1} D_{\perp}^{\prime} C_{1} \\
& H_{\infty 12}=\left(B_{1}-B_{2} D_{12}^{\prime} D_{11}\right)\left(\gamma^{2} I-D_{11}^{\prime} D_{\perp} D_{\perp}^{\prime} D_{11}\right)^{-1}\left(B_{1}-B_{2} D_{12}^{\prime} D_{11}\right)^{\prime}-B_{2} B_{2}^{\prime}
\end{aligned}
$$

(ii) Condition (ii) of Theorem 5.1 is satisfied with (5.8) replaced by

$$
J_{\infty}=\left[\begin{array}{cc}
J_{\infty 11} & J_{\infty 12} \\
J_{\infty 21} & -J_{\infty 11}^{\prime}
\end{array}\right]
$$

where

$$
\begin{aligned}
& J_{\infty 11}=A+B_{1}\left(\gamma^{2} I-\tilde{D}_{\perp}^{\prime} \tilde{D}_{\perp} D_{11}^{\prime} D_{11}\right)^{-1}\left(\tilde{D}_{\perp}^{\prime} \tilde{D}_{\perp} D_{11}^{\prime} C_{1}-\gamma^{2} D_{21}^{\prime} C_{2}\right), \\
& J_{\infty 21}=-B_{1} \tilde{D}_{\perp}^{\prime}\left(I-\gamma^{-2} \tilde{D}_{\perp} D_{11}^{\prime} D_{11} \tilde{D}_{\perp}^{\prime}\right)^{-1} \tilde{D}_{\perp} B_{1}^{\prime}, \\
& J_{\infty 12}=\left(C_{1}-D_{11} D_{21}^{\prime} C_{2}\right)^{\prime}\left(\gamma^{2} I-D_{11} \tilde{D}_{\perp}^{\prime} \tilde{D}_{\perp} D_{11}^{\prime}\right)^{-1}\left(C_{1}-D_{11} D_{21}^{\prime} C_{2}\right)-C_{2}^{\prime} C_{2} . \\
& {\left[\begin{array}{cc}
X_{\infty 2}^{\prime} X_{\infty 1} & \gamma^{-1} X_{\infty 22}^{\prime} Y_{\infty 2} \\
\gamma^{-1} Y_{\infty 2}^{\prime} X_{\infty 2} & Y_{\infty 2}^{\prime} Y_{\infty 1}
\end{array}\right] \geqq 0 .}
\end{aligned}
$$

All the solutions may be characterized by Theorem $5.2^{\prime}$.

THEOREM 5.2'. If the conditions of Theorem 5.1' are satisfied, then all internally stabilizing controllers satisfying $\left\|F_{l}(P, K)\right\|_{\infty} \leqq \gamma$ are given by

$$
K=F_{l}(\mathscr{K}, U) \text { with } U \in R H_{+}^{\infty}, \quad\|U\|_{\infty} \leqq \gamma
$$

where

$$
\begin{aligned}
& \mathscr{K}(s)=\left[\begin{array}{cc}
0 & D_{k 12} \\
D_{k 21} & D_{k 22}
\end{array}\right]+\left[\begin{array}{c}
C_{k 1} \\
C_{k 2}
\end{array}\right]\left(s E_{k}-A_{k}\right)^{\#}\left[\begin{array}{ll}
B_{k 1} & B_{k 2}
\end{array}\right], \\
& E_{k}=Y_{\infty 1}^{\prime} X_{\infty 1}-\gamma^{-2} Y_{\infty 2}^{\prime} X_{\infty 2}, \\
& B_{k 1}=\left(\gamma^{2} Y_{\infty 1}^{\prime} B_{1}+Y_{\infty 2}^{\prime} C_{1}^{\prime} D_{11}+Y_{\infty 2}^{\prime} C_{2}^{\prime} D_{21}\left(\gamma^{2} I-D_{11}^{\prime} D_{11}\right)\right)\left(\gamma^{2} I-\tilde{D}_{\perp}^{\prime} \tilde{D}_{\perp} D_{11}^{\prime} D_{11}\right)^{-1} D_{21}^{\prime}, \\
& C_{k 1}=-D_{12}^{\prime}\left(\gamma^{2} I-D_{11} D_{11}^{\prime} D_{\perp} D_{\perp}^{\prime}\right)^{-1}\left(\gamma^{2} C_{1} X_{\infty 1}+D_{11} B_{1}^{\prime} X_{\infty 2}\right. \\
&\left.+\left(\gamma^{2} I-D_{11} D_{11}^{\prime}\right) D_{12} B_{2}^{\prime} X_{\infty 2}\right), \\
& D_{k 12}=\left\{I-D_{12}^{\prime} D_{11}\left(\gamma^{2} I-D_{11}^{\prime} D_{\perp} D_{\perp}^{\prime} D_{11}\right)^{-1} D_{11}^{\prime} D_{12}\right\}^{1 / 2}, \\
& D_{k 21}=\left\{I-D_{21} D_{11}^{\prime}\left(\gamma^{2} I-D_{11} \tilde{D}_{\perp}^{\prime} \tilde{D}_{\perp} D_{11}^{\prime}\right)^{-1} D_{11} D_{21}^{\prime}\right\}^{1 / 2}, \\
& D_{k 22}=-\left(D_{k 21}^{-1}\right)^{\prime} D_{21} D_{11}^{\prime}\left(\gamma^{2} I-D_{11} \tilde{D}_{\perp}^{\prime} \tilde{D}_{\perp} D_{11}^{\prime}\right)^{-1} D_{12} D_{k 12}, \\
& A_{k}= E_{k} T_{x}+B_{k 1} D_{k 21}^{-1} C_{k 2} \\
&= T_{Y}^{\prime} E_{k}+B_{k 2} D_{k 12}^{-1} C_{k 1}, \\
& B_{k 2}=\{\left\{Y_{\infty 1}^{\prime} B_{2}+\left(Y_{\infty 1}^{\prime} B_{1} \tilde{D}_{\perp}^{\prime} \tilde{D}_{\perp} D_{11}^{\prime}+Y_{\infty 2}^{\prime}\left(C_{1}^{\prime}-C_{2}^{\prime} D_{21} D_{11}^{\prime}\right)\right)\right. \\
&\left.\cdot\left(\gamma^{2} I-D_{11} \tilde{D}_{\perp}^{\prime} \tilde{D}_{\perp} D_{11}^{\prime}\right)^{-1} D_{12}\right\} D_{k 12}, \\
& C_{k 2}=-D_{k 21}\left\{C_{2} X_{\infty 1}+D_{21}\left(\gamma^{2} I-D_{11}^{\prime} D_{\perp} D_{\perp}^{\prime} D_{11}\right)^{-1}\left(D_{11}^{\prime} D_{\perp} D_{\perp}^{\prime} C_{1} X_{\infty 1}+B_{1}^{\prime} X_{\infty 2}\right.\right. \\
&\left.\left.-D_{11}^{\prime} D_{12} B_{2}^{\prime} X_{\infty 2}\right)\right\} .
\end{aligned}
$$


6. Conclusions. The purpose of this paper was to derive a representation formula for all $H_{\infty}^{+}$solutions to the four block general distance problem in which particular attention was paid to the optimal cases. The suboptimal case was treated in $\S 3$, and was derived by an all-pass embedding procedure which is reminiscent of that introduced by Parrott [39]. The optimal case was treated in $\S 4$ and the formula for all solutions appears as (4.52) and (4.59). Section 5 dealt with the application to $H^{\infty}$ optimal control. In addition, we note the following:

(1) In contrast to the one block problem, there are two types of optimality. The first is essentially the same as that addressed in Parrott's theorem [39], and has nothing to do with the requirement that the approximation $Q$ be an element of $H_{\infty}^{+}$. The second is associated with a Hankel norm condition on $R_{a}$ in (3.46). In the first type of optimality, special algorithms may be required to take care of axis phenomena in the spectral factorization problems (3.18) and (3.19). These issues are addressed in detail in [8].

(2) The analysis in $\S 3$ breaks down when $\left\|R_{a}\right\|_{H}=1$ since $Z$ in (3.65) becomes singular. This difficulty is addressed in $\S 4$ where we introduce an alternative construction based on descriptor representations of all-pass transfer functions; see also [35] and [44] in this connection. In the case of this Hankel norm type of optimality, the effective dimension of the free parameter drops by rank $\left(B_{22}\right) \leqq k . B_{22}$ is defined in (4.38) and $k$ is the multiplicity of the largest Hankel singular value of $R_{a}$. This loss of effective dimension may be characterized by the linear constraining equation (4.45) which is similar to that given in [16, eq. (6.23)].

(3) An important application of this work is the derivation of a closed form representation formula for all controllers that satisfy an $L^{\infty}$ norm constraint. One such formula is given in $\S 5$ and appears in equations (5.10)-(5.18) in the case that $D_{11}=0$ and $D_{22}=0$ in the realization of $P(s)$ given in (5.1). All the optimal cases are covered.

(4) The case in which $D_{11} \neq 0$ has been treated by direct calculation and the results are given in Theorem 5.2'. Since these formulae are awkward to write down, an alternative approach based on loop shifting is also presented [45]. The idea here is to replace the original problem which has $D_{11} \neq 0$ with an equivalent problem in which $\hat{D}_{11}=0$.

\section{REFERENCES}

[1] B. D. O. ANDERSOn, An algebraic solution to the spectral factorization problem, IEEE Trans. Automat. Control, 12 (1967), pp. 410-414.

[2] B. D. O. Anderson And S. Vongpanitlerd, Network Analysis and Synthesis a Modern Systems Approach, Prentice-Hall, Englewood Cliffs, NJ, 1973.

[3] J. A. BAll AND N. COHEN, Sensitivity minimization in an $H^{\infty}$ norm: parameterization of all suboptimal solutions, Internat. J. Control, 46 (1987), pp. 785-816.

[4] M. BANKer, Linear stationary quadratic games, in Proc. Conference on Decision and Control, 1973, pp. 193-197.

[5] D. S. BERNSTEIN AND W. M. HADDAD, LQG control with an $H^{\infty}$ performance bound: a Riccati equation approach, IEEE Trans. Automat. Control, 34 (1989), pp. 293-305.

[6] M. Bettayeb, L. M. Silverman, And M. G. Safonov, Optimal approximation of continuous time systems, in Proc. IEEE Conference on Decision and Control, Albuquerque, NM, December 10-12, 1980.

[7] C. C. Chu, J. C. Doyle, AND E. B. LeE, The general distance problem in $H^{\infty}$ optimal control theory, Internat. J. Control, 44 (1986), pp. 565-596.

[8] D. J. Clements AND K. Glover, Spectral factorization via Hermitian pencils, Linear Algebra Appl., Vol. 122/123/124 (1989), pp. 797-846. 
[9] C. Davis, W. M. Kahan, AND H. F. Weinberger, Norm-preserving dilations and their applications to optimal error bounds, SIAM J. Control Optim., (1982), pp. 445-469.

[10] J. C. DOYLE et al, Lecture notes in advances in multivariable control, ONR/Honeywell Workshop, Minneapolis, MN, 1984.

[11] J. C. Doyle, K. Glover, P. Khargonekar, And B. Francis, State-space solutions to standard $\mathrm{H}_{2}$ and $\mathrm{H}_{\infty}$ control problems IEEE American Control Conference, Atlanta, GA, June 1988; for an extended version see IEEE Trans. Automat. Control, 34 (1989), pp. 831-847.

[12] H. DYM AND I. GoHBERG, A new class of contractive interpolants and maximum entropy principles, Birkhauser-Verlag, Berlin, 1988, pp. 117-150.

[13a] C. Foias And A. TAnnenbaum, On the four block problem I, Operator Theory Adv. and Appl., 32 (1988), pp. 93-122.

[13b] - On the four block problem II: The singular system, Integral Equations and Operator Theory, 11 (1988), pp. 726-767.

[14] B. A. Francis, $A$ course in $H^{\infty}$ control theory, Lecture Notes in Control and Information Sciences, Vol. 88, Springer-Verlag, Berlin, New York, 1987.

[15] B. A. FrAnCIS AND J. C. DoYle, Linear control theory with an $\boldsymbol{H}^{\infty}$ optimality criterion, SIAM J. Control Optim., 25 (1987), pp. 815-844.

[16] K. Glover, All optimal Hankel-norm approximations of linear multivariable systems and their $L^{\infty}$-error bounds, Internat. J. Control, 39 (1984), pp. 1115-1193.

[17] - Model reduction: A tutorial on Hankel-norm methods and lower bounds on $L^{2}$ errors, IFAC Congress, Munich, July, 1987.

[18] — A tutorial on Hankel norm approximation, in From Data to Model, J. C. Willems, ed., SpringerVerlag, Berlin, New York, 1989, pp. 26-48.

[19] K. Glover, R. F. Curtain, ANd J. R. PARtington, Realisation and approximation of linear infinite dimensional systems with error bounds, SIAM J. Control Optim., 26 (1988), pp. 863-898.

[20] K. Glover AND J. C. DOYLE, State-space formulae for all stabilizing controllers that satisfy a $\mathrm{H}^{\infty}$ norm bound and relations to risk sensitivity, Systems Control Lett., 11 (1988), pp. 167-172.

[21] K. Glover AND D. Mustafa, Derivation of the maximum entropy $H^{\infty}$ controller and a state-space formula for its entropy, Internat. J. Control, 50 (1989), pp. 899-916.

[22] M. Green, K. Glover, D. J. N. Limebeer, And J. C. Doyle, A j-spectral factorization approach to $H_{\infty}$ control, SIAM J. Control Optim., 28 (1990), pp. 1350-1371.

[23] Y.S. HUNG, $H^{\infty}$ optimal control: part I model matching, Internat. J. Control, 49 (1989), pp. 1331-1359.

[24] —,$H^{\infty}$ optimal control-Part II solutions for controllers, Internat. J. Control, 49 (1989), pp. 1331-1359.

[25] D. H. JACOBSON, Optimal stochastic linear systems with exponential performance criteria and their relation to deterministic differential games, IEEE Trans. Automat. Control, 18 (1973), pp. 124-131.

[26] E. A. JONCKHEERE AND J. C. JUANG, Fast computation of achievable performance in mixed sensitivity $H^{\infty}$ design, IEEE Trans. Automat. Control., 32 (1987), pp. 896-906.

[27] E. M. KASENALLy AND D. J. N. LIMEBEER, Closed formulae for a parametric mixed sensitivity problem, Systems Control Lett., 12 (1989), pp. 1-7.

[28] P. P. Khargonekar, I. R. Petersen, And M. A. Rotea, $H^{\infty}$ optimal control with state feedback, IEEE Trans. Automat. Control, 33 (1988), pp. 783-786.

[29] H. KIMURA AND R. KAWATANi, Synthesis of $H^{\infty}$ controllers based on conjugation, Proc. IEEE Conference on Decision and Control, Austin, TX, 1988.

[30] D. J. N. Limebeer AND B. D. O. Anderson, An interpolation theory approach to $H^{\infty}$ controller degree bounds, Linear Algebra Appl., 98 (1988), pp. 347-386.

[31] D. J. N. Limebeer AND G. D. HAlikiAs, An analysis of pole zero cancellations in $H^{\infty}$-optimal control problems of the second kind, SIAM J. Control Optim., 26 (1988), pp. 646-677.

[32] D. J. N. Limebeer AND Y. S. HUNG, An analysis of pole-zero cancellations in $H^{\infty}$-optimal control problems of the first kind, SIAM. J. Control Optim., 25 (1987), pp. 1457-1493.

[33] D. J. N. Limebeer ANd E. M. KASEnAlly, Vector interpolation: Applications to $H^{\infty}$, IMA Conference on Matrix Theory, Bradford, July 1988.

[34] D. J. N. Limebeer, B. D. O. Anderson, P. P. Khargonekar, And M. Green, A game theoretic approach to $H^{\infty}$ control for time varying systems, SIAM J. Control Optim., submitted.

[35] D. J. N. Limebeer, E. M. Kasenally, E. Jaimouka, And M. G. SAfonov, A characterization of all solutions to the four block general distance problem, in Proc. IEEE Conference on Decision and Control, Austin, TX, 1988.

[36] D. J. N. Limebeer, G. D. Halikias, AND K. Glover, A state-space algorithm for the computation of superoptimal matrix interpolating functions, Linear Circuits, Systems and Signal Processing: Theory and Applications, North-Holland, Amsterdam, 1988. 
[37] D. G. Luenberger, Dynamic equations in descriptor form, IEEE Trans. Automat. Control, 22 (1977), pp. 312-321.

[38] D. Mustafa, K. Glover, And D. J. N. Limebeer, Controllers which satisfy a closed-loop $H^{\infty}$-norm bound and maximize an entropy integral, Automatica, to appear.

[39] S. PARrott, On a quotient norm and the Sz.-Nagy-Foias lifting theorem, J. Func. Anal., 30 (1978), pp. 311-328.

[40] I. R. Petersen AND D. J. Clements, J-spectral factorization and Riccati equations in problems of $H^{\infty}$ optimization via state feedback, preprint, 1988.

[41] S. C. Power, Hankel Operators on Hilbert Space, Pitman Advanced Publishing Program, Boston, 1981.

[42] R. M. REDHEFFER, On a certain linear fractional transformation, J. Math. Phys., 39 (1960), pp. 269-286.

[43] M. G. Safonov, E. A. Jonckheere, M. Verma, And D. J. N. Limebeer, Systhesis of positive real multivariable feedback systems, Internat. J. Control, 45 (1987), pp. 817-842.

[44] M. G. SAFonov, R. Y. ChIANG, AND D. J. N. LimebeER, Hankel model reduction without balancing: A descriptor system approach, IEEE Conference on Decision and Control, Los Angeles, CA, 1987; an extended version to appear in IEEE Trans. Automat. Control.

[45] M. G. Safonov, D. J. N. Limebeer, And R. Y. Chiang Simplifying the $H^{\infty}$ theory via loop shifting, matrix pencil and descriptor concepts, Internat. J. Control., 50 (1990), pp. 2467-2488.

[46] M. G. SAFONOV AND M. Verma, Multivariable $L^{\infty}$ sensitivity optimization and Hankel approximation, in Proc. American Control Conference, San Francisco, CA, 1983; also in IEEE Trans. Automat. Control, 30 (1985), pp. 279-280.

[47] G. TADMOR, Worst-case design in the time domain; the maximum principle and the standard $H_{\infty}$ problem, Math. of Control Signals and Systems, to appear.

[48] M. S. Verma AND J. C. Romig, Reduced order controllers in $H^{\infty}$-optimal synthesis methods of the first kind, December, 1987, submitted.

[49] P. WhitTle, Risk sensitive linear quadratic gaussian control, Adv. Appl. Probab., 13 (1981), pp. $764-777$.

[50] J. C. WILLEMS, Least squares stationary optimal control and the algebraic Riccati equation, IEEE Trans. Automat. Control, 16 (1971), 621-634.

[51] H. Wimmer, Monotonicity of maximal solutions of algebraic Riccati equations, Systems Control Lett., 5 (1985), pp. 317-319.

[52] W. M. Wonham, Linear Multivariable Control: A Geometric Approach, Series on Applications of Mathematics, Springer-Verlag, Berlin, New York, 1979.

[53] N. Young, An Introduction to Hilbert Space, Cambridge University Press, London, 1988. 